\title{
Metal-Free Intramolecular Aminophosphination of Allenes
}

Zhantao Yang, ${ }^{\text {a,b }}$ Zhenzhen Sun, ${ }^{a}$ Ningning Zhu, ${ }^{a}$ Wenyan Li, ${ }^{a}$ Meng Han, ${ }^{a}$ Yan Qiao, ${ }^{c}$ Chun-Hua Yang $*^{\mathrm{a}}$ and Junbiao Chang $*^{\mathrm{b}}$

\footnotetext{
${ }^{a}$ Henan Province Key Laboratory of New Opto-Electronic Functional Materials, College of Chemistry and Chemical Engineering, Anyang Normal University, 436 Xian'ge Road, Anyang 455000, P. R. China.

${ }^{\mathrm{b}}$ School of Pharmaceutical Sciences, Zhengzhou University, Zhengzhou, Henan 450001, P. R. China.

${ }^{\mathrm{c}}$ School of Basic Medicine, Zhengzhou University, Zhengzhou, Henan 450001, P. R. China.
}

\section{Table of Contents}

$\begin{array}{ll}\text { 1. Calculation results } & \mathrm{S} 1\end{array}$

$\begin{array}{ll}\text { 2. References } & \text { S17 }\end{array}$

$\begin{array}{lr}\text { 3. Copies of NMR Spectra } & \text { S18 }\end{array}$

4. X-ray Crystal Structure and Data of $\mathbf{5 g} \quad$ S51 


\section{Calculation results}

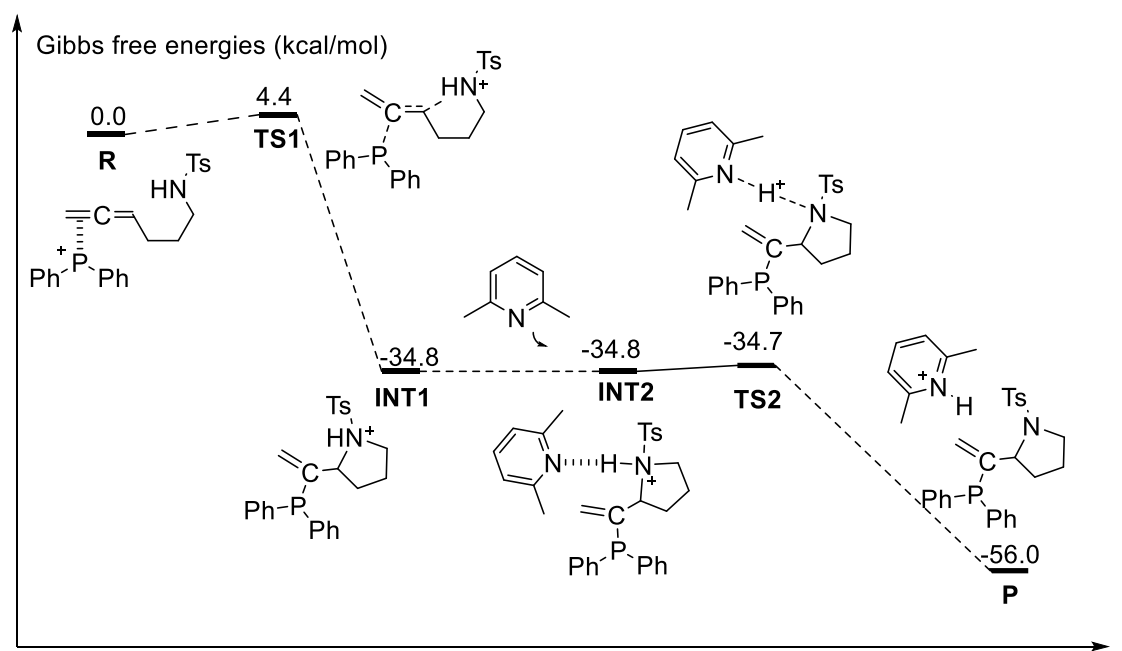

Figure S1. Gibbs energy profile for the transformation from $(\mathrm{Ph})_{2} \mathrm{P}^{+}$and allene to the five-membered ring product

To shed light on the detailed mechanism for the formation of five-membered ring, DFT calculations were performed at the M06-2X/6-31G(d,p) level as follows. As shown in Figure S1, there are two steps involved in the transformation from $(\mathrm{Ph})_{2} \mathrm{P}^{+}$and allene to the five-membered ring product. The first step is the C-P bond formation coupled with the C-N bond formation to afford intermediate INT1 via transition state TS1 with an energy barrier of $4.4 \mathrm{kcal} / \mathrm{mol}$. The second step is the proton abstraction by 1,6-dimethylpyridine via transition state TS2 to generate the final five-membered ring product. The free energy barrier for TS2 is only $0.1 \mathrm{kcal} / \mathrm{mol}$, indicating that it is a facile reaction process.

As shown, the first reaction step is stereoselective-determining. In experiments, the reactant $\mathbf{4 g}$ can stereoselectively lead to the anti- product. To ascertain the stereoselectivity-determining factors, we also calculated the ring-closer transition states $\mathrm{TS}^{\text {anti }}$ and $\mathrm{TS}^{\text {syn }}$. The anti-transition state was calculated to be $2.1 \mathrm{kcal} / \mathrm{mol}$ lower than the syn-transition state, which can explain the experimental results that the anti-product is the major product observed. Structural comparison reveals that the

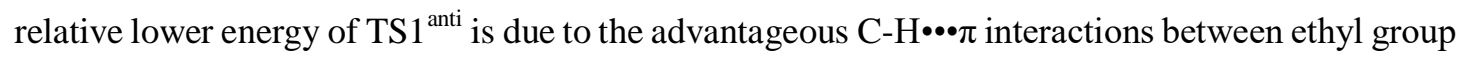
and the two phenyl groups, which is absent in $\mathrm{TS}^{\mathrm{syn}}$.

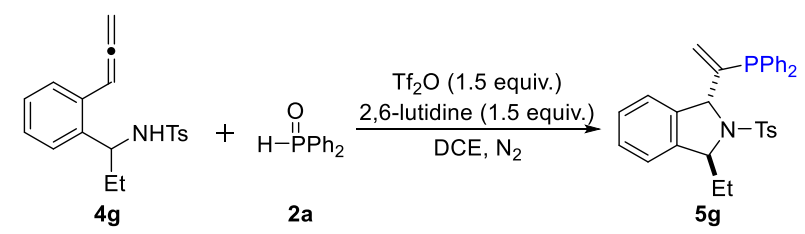



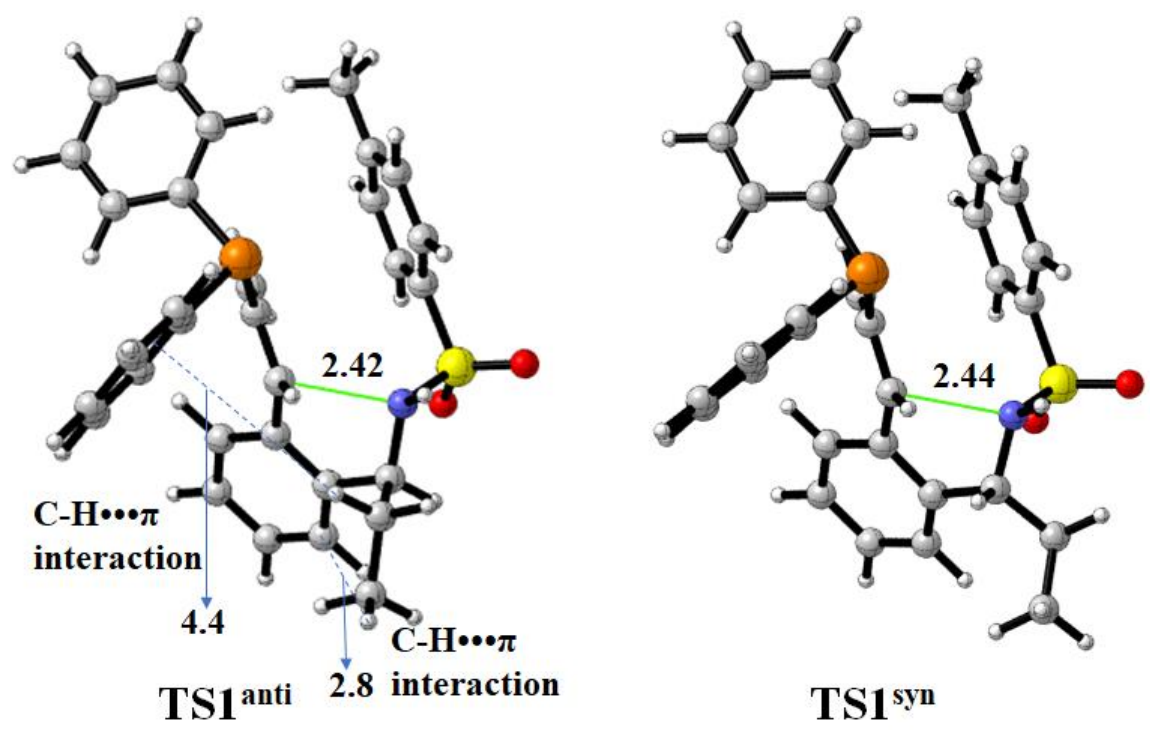

TS1 ${ }^{\text {syn }}$

\section{Computational Methods:}

The DFT calculations were performed using the Gaussian 09 program. The geometries were fully optimized using M06-2X functional ${ }^{1}$ and 6-31G(d, p) basis set (B1 level). The corresponding frequency calculations at the same level of theory were also carried out to identify all of the stationary points as minima (zero imaginary frequency) or transition state (only one imaginary frequency), and to provide corrections for free energies. Then, single-point energy refinements in solvent dichloroethane were carried out using M06-2X functional with 6-311+G(d, p) basis set (B2 level). Finally, the Gibbs free energies at the M06-2X/6-311+G(d, p) level, which is evaluated as the sum of the single-point energy at M06-2X/6-311+G(d, p) level and the thermal correction at M06-2X/6-31G(d, p) level, were used in the following discussion.

\section{Energies and Geometrical Coordinates of the Listed Complexes}

$\mathrm{R}$

Zero-point correction=

Thermal correction to Energy=

Thermal correction to Enthalpy=

Thermal correction to Gibbs Free Energy=

Sum of electronic and zero-point Energies=

Sum of electronic and thermal Energies=

Sum of electronic and thermal Enthalpies=

Sum of electronic and thermal Free Energies=

$\mathrm{E} / \mathrm{B} 2=-1913.13487$

11

$\mathrm{C}$

0.99631900
0.467639 (Hartree/Particle)

0.496821

0.497765

0.403132

$-1912.270613$

$-1912.241432$

$-1912.240488$

$-1912.335120$ 


\begin{tabular}{|c|c|c|c|}
\hline C & 0.80426500 & 2.58721800 & -0.51694300 \\
\hline $\mathrm{C}$ & 0.66674800 & 2.05530900 & -1.95279500 \\
\hline $\mathrm{C}$ & 1.70261400 & 1.00517000 & -2.36505400 \\
\hline $\mathrm{N}$ & 1.30735500 & -0.31596000 & -1.85182500 \\
\hline $\mathrm{H}$ & -0.07907200 & 3.17789300 & -0.24900100 \\
\hline $\mathrm{H}$ & 0.74771900 & 2.91776200 & -2.62149400 \\
\hline $\mathrm{H}$ & 2.67920500 & 1.26024600 & -1.94279500 \\
\hline $\mathrm{H}$ & -0.33427500 & 1.63871000 & -2.10432400 \\
\hline $\mathrm{H}$ & 1.82538400 & 0.98338800 & -3.45187000 \\
\hline $\mathrm{H}$ & 1.67295000 & 3.24613000 & -0.44978600 \\
\hline $\mathrm{H}$ & 1.86448300 & 1.55856500 & 1.21888700 \\
\hline $\mathrm{C}$ & 0.27798600 & 0.45743000 & 0.71217200 \\
\hline $\mathrm{C}$ & -0.48680500 & -0.68085100 & 0.75553600 \\
\hline $\mathrm{H}$ & -0.00727600 & -1.57167300 & 0.33413700 \\
\hline $\mathrm{H}$ & -1.07528600 & -0.83070700 & 1.66121300 \\
\hline$P$ & -1.93324200 & -0.31341600 & -0.67658800 \\
\hline $\mathrm{C}$ & -2.45902300 & 1.37568400 & -0.20760900 \\
\hline $\mathrm{C}$ & -2.86522300 & 2.19835200 & -1.26551800 \\
\hline $\mathrm{C}$ & -2.46567800 & 1.88440400 & 1.09840400 \\
\hline $\mathrm{C}$ & -3.27542900 & 3.50724000 & -1.02299500 \\
\hline $\mathrm{H}$ & -2.85992900 & 1.81687500 & -2.28408100 \\
\hline $\mathrm{C}$ & -2.86137300 & 3.19583700 & 1.33619500 \\
\hline $\mathrm{H}$ & -2.16644100 & 1.25915600 & 1.93617200 \\
\hline $\mathrm{C}$ & -3.26627100 & 4.00740700 & 0.27566900 \\
\hline $\mathrm{H}$ & -3.59413000 & 4.13426400 & -1.84857800 \\
\hline $\mathrm{H}$ & -2.85963900 & 3.58468900 & 2.34895900 \\
\hline $\mathrm{H}$ & -3.57618900 & 5.02961400 & 0.46567400 \\
\hline $\mathrm{C}$ & -3.16502400 & -1.40451800 & 0.09505400 \\
\hline C & -3.02499700 & -2.77139300 & -0.19541400 \\
\hline $\mathrm{C}$ & -4.23141400 & -0.96633900 & 0.89103500 \\
\hline $\mathrm{C}$ & -3.92996900 & -3.68980300 & 0.32198600 \\
\hline $\mathrm{H}$ & -2.21101800 & -3.11429200 & -0.83102900 \\
\hline $\mathrm{C}$ & -5.14118900 & -1.89069400 & 1.39275000 \\
\hline $\mathrm{H}$ & -4.36311200 & 0.08991000 & 1.10226500 \\
\hline $\mathrm{C}$ & -4.98719800 & -3.24798900 & 1.11580000 \\
\hline $\mathrm{H}$ & -3.81762300 & -4.74504000 & 0.09845800 \\
\hline $\mathrm{H}$ & -5.97428500 & -1.55180200 & 1.99910500 \\
\hline $\mathrm{H}$ & -5.69933200 & -3.96358800 & 1.51308900 \\
\hline$S$ & 2.50857900 & -1.45836300 & -1.56121800 \\
\hline $\mathrm{C}$ & 3.23247400 & -0.85353900 & -0.05559500 \\
\hline $\mathrm{C}$ & 4.19990400 & 0.14620700 & -0.10808700 \\
\hline $\mathrm{C}$ & 2.79034100 & -1.36534800 & 1.16205500 \\
\hline C & 4.67500900 & 0.68706800 & 1.08337800 \\
\hline $\mathrm{H}$ & 4.59359800 & 0.47327000 & -1.06562200 \\
\hline
\end{tabular}




$\begin{array}{lrrr}\mathrm{C} & 3.28498800 & -0.82269400 & 2.34309000 \\ \mathrm{H} & 2.09472700 & -2.19841600 & 1.17292600 \\ \mathrm{C} & 4.22143200 & 0.21863800 & 2.32280700 \\ \mathrm{H} & 5.43043100 & 1.46715100 & 1.05262600 \\ \mathrm{H} & 2.95743000 & -1.22523400 & 3.29749100 \\ \mathrm{C} & 4.78271200 & 0.77306100 & 3.60471200 \\ \mathrm{H} & 5.67651800 & 0.21229500 & 3.89534200 \\ \mathrm{H} & 5.07256900 & 1.81995700 & 3.49384700 \\ \mathrm{H} & 4.06371600 & 0.69386100 & 4.42262600 \\ \mathrm{O} & 3.52481300 & -1.41054000 & -2.59547900 \\ \mathrm{O} & 1.75060800 & -2.66767600 & -1.26382800 \\ \mathrm{H} & 0.59420300 & -0.77453000 & -2.41942800\end{array}$

TS1

\begin{tabular}{|c|c|c|c|}
\hline \multicolumn{3}{|c|}{ Zero-point correction= } & 0.468372 (Hartree/Particle) \\
\hline \multicolumn{3}{|c|}{ Thermal correction to Energy= } & 0.496578 \\
\hline \multicolumn{3}{|c|}{ Thermal correction to Enthalpy= } & 0.497522 \\
\hline \multicolumn{3}{|c|}{ Thermal correction to Gibbs Free Energy= } & 0.407925 \\
\hline \multicolumn{3}{|c|}{ Sum of electronic and zero-point Energies= } & -1912.270160 \\
\hline \multicolumn{3}{|c|}{ Sum of electronic and thermal Energies= } & -1912.241954 \\
\hline \multicolumn{3}{|c|}{ Sum of electronic and thermal Enthalpies= } & -1912.241010 \\
\hline \multicolumn{3}{|c|}{ Sum of electronic and thermal Free Energies= } & -1912.330607 \\
\hline \multicolumn{4}{|c|}{$\mathrm{E} / \mathrm{B} 2=-1913.132691$} \\
\hline \multicolumn{4}{|l|}{11} \\
\hline $\mathrm{C}$ & -0.44721800 & 0.93707400 & 1.57450800 \\
\hline $\mathrm{C}$ & -0.42974700 & 0.54071300 & 3.01299300 \\
\hline $\mathrm{C}$ & -1.30811200 & -0.68831900 & 3.35975500 \\
\hline $\mathrm{C}$ & -2.59082900 & -0.81035200 & 2.54250600 \\
\hline $\mathrm{N}$ & -2.22943800 & -1.30051600 & 1.20578500 \\
\hline $\mathrm{H}$ & 0.59150200 & 0.36877300 & 3.35779100 \\
\hline $\mathrm{H}$ & -1.55086200 & -0.64369900 & 4.42412400 \\
\hline $\mathrm{H}$ & -3.07960800 & 0.16483100 & 2.42896900 \\
\hline $\mathrm{H}$ & -0.71527400 & -1.59909300 & 3.21261700 \\
\hline $\mathrm{H}$ & -3.31230000 & -1.47253100 & 3.03242400 \\
\hline $\mathrm{H}$ & -0.81821000 & 1.40691300 & 3.56344400 \\
\hline $\mathrm{H}$ & -1.36721300 & 1.39023200 & 1.20070300 \\
\hline $\mathrm{C}$ & 0.51098000 & 0.76447000 & 0.64511900 \\
\hline $\mathrm{C}$ & 0.78944600 & 1.26389800 & -0.60824800 \\
\hline $\mathrm{H}$ & 1.05668200 & 0.59560000 & -1.42483400 \\
\hline $\mathrm{H}$ & 0.88677100 & 2.33491500 & -0.78598600 \\
\hline $\mathrm{P}$ & 2.03934400 & -0.34826000 & 0.94731600 \\
\hline $\mathrm{C}$ & 3.56639300 & 0.53950500 & 0.47798600 \\
\hline $\mathrm{C}$ & 4.29075000 & 1.10374600 & 1.53376100 \\
\hline $\mathrm{C}$ & 4.04908400 & 0.64917700 & -0.83336200 \\
\hline
\end{tabular}




\begin{tabular}{lrrr}
$\mathrm{C}$ & 5.47874700 & 1.78630000 & 1.28016300 \\
$\mathrm{H}$ & 3.93016400 & 1.00496400 & 2.55418200 \\
$\mathrm{C}$ & 5.23598000 & 1.32576900 & -1.07933000 \\
$\mathrm{H}$ & 3.50787100 & 0.18902100 & -1.65596900 \\
$\mathrm{C}$ & 5.94899700 & 1.89650800 & -0.02338800 \\
$\mathrm{H}$ & 6.03610900 & 2.22258200 & 2.10229100 \\
$\mathrm{H}$ & 5.60961400 & 1.40938100 & -2.09436100 \\
$\mathrm{H}$ & 6.87695700 & 2.42324200 & -0.22173400 \\
$\mathrm{C}$ & 1.79635000 & -1.64401800 & -0.30008600 \\
$\mathrm{C}$ & 0.49591900 & -1.98979800 & -0.69780900 \\
$\mathrm{C}$ & 2.88753100 & -2.38397700 & -0.77659700 \\
$\mathrm{C}$ & 0.29045100 & -3.04415800 & -1.58208100 \\
$\mathrm{H}$ & -0.36067200 & -1.43798700 & -0.32317000 \\
$\mathrm{C}$ & 2.67196100 & -3.44522100 & -1.64915400 \\
$\mathrm{H}$ & 3.89881400 & -2.13474200 & -0.46941200 \\
$\mathrm{C}$ & 1.37945700 & -3.77331400 & -2.05599800 \\
$\mathrm{H}$ & -0.72494300 & -3.28820200 & -1.88080300 \\
$\mathrm{H}$ & 3.51845700 & -4.01714700 & -2.01433400 \\
$\mathrm{H}$ & 1.22108600 & -4.59933500 & -2.74118500 \\
$\mathrm{~S}$ & -3.37008800 & -1.19257700 & -0.02676000 \\
$\mathrm{C}$ & -3.07300500 & 0.44952300 & -0.63326200 \\
$\mathrm{C}$ & -3.78152300 & 1.52027100 & -0.09594300 \\
$\mathrm{C}$ & -2.10081700 & 0.63781000 & -1.61219100 \\
$\mathrm{C}$ & -3.47015500 & 2.81080400 & -0.51579000 \\
$\mathrm{H}$ & -4.57467800 & 1.33663500 & 0.62350600 \\
$\mathrm{C}$ & -1.81415900 & 1.93538600 & -2.03057800 \\
$\mathrm{H}$ & -1.60384300 & -0.22301900 & -2.05026100 \\
$\mathrm{C}$ & -2.48349900 & 3.03793300 & -1.48069000 \\
$\mathrm{H}$ & -4.01665700 & 3.65422400 & -0.10344300 \\
$\mathrm{H}$ & -1.08762500 & 2.09606500 & -2.82360000 \\
$\mathrm{C}$ & -2.19509900 & 4.43283400 & -1.96799000 \\
$\mathrm{H}$ & -2.88806000 & 4.70009500 & -2.77341200 \\
$\mathrm{H}$ & -2.32008500 & 5.16760100 & -1.16903000 \\
$\mathrm{H}$ & -1.18005500 & 4.51712300 & -2.36617400 \\
$\mathrm{H}$ & -1195900 & -1.22872700 & 0.52493000 \\
$\mathrm{H}$ & -2.14722200 & -1.01788000 \\
$\mathrm{H}$ & -2.26546100 & 1.21340300 \\
\hline
\end{tabular}

INT1

$\begin{array}{lc}\text { Zero-point correction }= & 0.473031(\text { Hartree/Particle }) \\ \text { Thermal correction to Energy= } & 0.500766 \\ \text { Thermal correction to Enthalpy= } & 0.501710 \\ \text { Thermal correction to Gibbs Free Energy= } & 0.411577 \\ \text { Sum of electronic and zero-point Energies= } & -1912.338620 \\ & \text { S5 }\end{array}$




\begin{tabular}{|c|c|c|c|}
\hline \multicolumn{3}{|c|}{ Sum of electronic and thermal Energies= } & -1912.310 \\
\hline \multicolumn{3}{|c|}{ Sum of electronic and thermal Enthalpies= } & -1912.309 \\
\hline \multicolumn{3}{|c|}{ Sum of electronic and thermal Free Energies= } & -1912.400 \\
\hline \multicolumn{4}{|c|}{$\mathrm{E} / \mathrm{B} 2=-1913.1988$} \\
\hline \multicolumn{4}{|c|}{11} \\
\hline $\mathrm{C}$ & -0.55350300 & -0.69612500 & 0.52138600 \\
\hline $\mathrm{C}$ & -0.33334700 & -2.22622000 & 0.67134300 \\
\hline $\mathrm{C}$ & -0.41463800 & -2.80762600 & -0.74652700 \\
\hline $\mathrm{C}$ & -1.41856200 & -1.89551200 & -1.43896900 \\
\hline $\mathrm{N}$ & -0.99840900 & -0.54623900 & -0.93699800 \\
\hline $\mathrm{H}$ & 0.62378000 & -2.43392100 & 1.15212000 \\
\hline $\mathrm{H}$ & -0.73846500 & -3.84899600 & -0.76034800 \\
\hline $\mathrm{H}$ & -2.43845400 & -2.07250400 & -1.08315800 \\
\hline $\mathrm{H}$ & 0.55792900 & -2.74608400 & -1.24663100 \\
\hline $\mathrm{H}$ & -1.39659100 & -1.89205900 & -2.52840700 \\
\hline $\mathrm{H}$ & -1.12738300 & -2.64012900 & 1.29759400 \\
\hline $\mathrm{H}$ & -1.39454400 & -0.36915900 & 1.13976500 \\
\hline $\mathrm{C}$ & 0.64966300 & 0.18474500 & 0.77914700 \\
\hline $\mathrm{C}$ & 0.66888200 & 0.98339200 & 1.84590600 \\
\hline $\mathrm{H}$ & -0.17495800 & 1.03166500 & 2.53209800 \\
\hline $\mathrm{H}$ & 1.52022900 & 1.62119200 & 2.06384300 \\
\hline $\mathrm{P}$ & 1.95454000 & 0.12378200 & -0.53591800 \\
\hline $\mathrm{C}$ & 2.95119600 & -1.32648300 & -0.02029200 \\
\hline $\mathrm{C}$ & 3.44853600 & -2.16527600 & -1.02264200 \\
\hline $\mathrm{C}$ & 3.23869300 & -1.61176700 & 1.32136400 \\
\hline $\mathrm{C}$ & 4.21986600 & -3.27799300 & -0.69077900 \\
\hline $\mathrm{H}$ & 3.23621900 & -1.94321300 & -2.06554400 \\
\hline $\mathrm{C}$ & 4.00585200 & -2.72416900 & 1.65022400 \\
\hline $\mathrm{H}$ & 2.85934600 & -0.95966100 & 2.10450700 \\
\hline $\mathrm{C}$ & 4.49498200 & -3.55786000 & 0.64404800 \\
\hline $\mathrm{H}$ & 4.60493300 & -3.92278200 & -1.47359700 \\
\hline $\mathrm{H}$ & 4.22688500 & -2.94047900 & 2.69033900 \\
\hline $\mathrm{H}$ & 5.09293200 & -4.42495600 & 0.90423500 \\
\hline $\mathrm{C}$ & 2.97125700 & 1.58782700 & -0.13796400 \\
\hline $\mathrm{C}$ & 2.40193500 & 2.84685800 & -0.37239300 \\
\hline $\mathrm{C}$ & 4.28389000 & 1.49580200 & 0.32824700 \\
\hline $\mathrm{C}$ & 3.13520200 & 3.99974900 & -0.12122500 \\
\hline $\mathrm{H}$ & 1.38244400 & 2.92277100 & -0.74446000 \\
\hline $\mathrm{C}$ & 5.01771400 & 2.65669100 & 0.56609500 \\
\hline $\mathrm{H}$ & 4.73899600 & 0.52526500 & 0.49891400 \\
\hline $\mathrm{C}$ & 4.44556800 & 3.90517600 & 0.34638400 \\
\hline $\mathrm{H}$ & 2.68831500 & 4.97233300 & -0.29848300 \\
\hline $\mathrm{H}$ & 6.03893700 & 2.58107100 & 0.92439400 \\
\hline $\mathrm{H}$ & 5.02010500 & 4.80611900 & 0.53419500 \\
\hline
\end{tabular}




$\begin{array}{lrrr}\mathrm{S} & -2.14141900 & 0.83715900 & -1.30932300 \\ \mathrm{C} & -3.55121600 & 0.48406100 & -0.33528400 \\ \mathrm{C} & -4.56981600 & -0.29573800 & -0.88728300 \\ \mathrm{C} & -3.65076200 & 1.04704800 & 0.93960300 \\ \mathrm{C} & -5.69962400 & -0.53864800 & -0.12013600 \\ \mathrm{H} & -4.48446100 & -0.67349700 & -1.90144100 \\ \mathrm{C} & -4.79305000 & 0.78880100 & 1.68270800 \\ \mathrm{H} & -2.86187700 & 1.69136800 & 1.31632200 \\ \mathrm{C} & -5.82785800 & -0.00463500 & 1.16898800 \\ \mathrm{H} & -6.50485900 & -1.13891900 & -0.53194600 \\ \mathrm{H} & -4.89495700 & 1.22142800 & 2.67307600 \\ \mathrm{C} & -7.07631400 & -0.23974600 & 1.97145200 \\ \mathrm{H} & -7.79764100 & 0.56156100 & 1.78070500 \\ \mathrm{H} & -7.55109900 & -1.18463000 & 1.70151200 \\ \mathrm{H} & -6.86459600 & -0.24634200 & 3.04225000 \\ \mathrm{O} & -2.40530500 & 0.61392000 & -2.70916700 \\ \mathrm{O} & -1.41478700 & 1.96875100 & -0.78819600 \\ \mathrm{H} & -0.13423500 & -0.26052700 & -1.45643400\end{array}$

1,6-dimethylpyridine

\begin{tabular}{|c|c|c|c|}
\hline \multicolumn{3}{|c|}{ Zero-point correction= } & 0.145140 (Hartree/Particle) \\
\hline \multicolumn{3}{|c|}{ Thermal correction to Energy= } & 0.152766 \\
\hline \multicolumn{3}{|c|}{ Thermal correction to Enthalpy= } & 0.153710 \\
\hline \multicolumn{3}{|c|}{ Thermal correction to Gibbs Free Energy= } & 0.113091 \\
\hline \multicolumn{3}{|c|}{ Sum of electronic and zero-point Energies= } & -326.636389 \\
\hline \multicolumn{3}{|c|}{ Sum of electronic and thermal Energies= } & -326.628763 \\
\hline \multicolumn{3}{|c|}{ Sum of electronic and thermal Enthalpies= } & -326.627819 \\
\hline \multicolumn{3}{|c|}{ Sum of electronic and thermal Free Energies= } & -326.668437 \\
\hline \multicolumn{4}{|c|}{$\mathrm{E} / \mathrm{B} 2=-326.8626264$} \\
\hline \multicolumn{4}{|c|}{01} \\
\hline $\mathrm{C}$ & -0.18688200 & 1.21508000 & -1.13142700 \\
\hline $\mathrm{C}$ & 1.08488000 & 1.06685400 & -0.59301200 \\
\hline $\mathrm{C}$ & 1.23458900 & 0.31800100 & 0.57624100 \\
\hline $\mathrm{C}$ & -1.02576900 & -0.11583700 & 0.67590300 \\
\hline $\mathrm{C}$ & -1.26317700 & 0.61569700 & -0.48987300 \\
\hline $\mathrm{H}$ & -0.33783100 & 1.79053900 & -2.03992800 \\
\hline $\mathrm{H}$ & 1.95060800 & 1.52038500 & -1.06381400 \\
\hline $\mathrm{H}$ & -2.27149200 & 0.70873800 & -0.87900200 \\
\hline $\mathrm{N}$ & 0.19857300 & -0.25900600 & 1.19412800 \\
\hline $\mathrm{C}$ & 2.58290700 & 0.11088500 & 1.21298900 \\
\hline $\mathrm{H}$ & 3.36896400 & 0.64910600 & 0.67986700 \\
\hline $\mathrm{H}$ & 2.56139800 & 0.44962300 & 2.25172500 \\
\hline $\mathrm{H}$ & 2.83121500 & -0.95381700 & 1.22468600 \\
\hline $\mathrm{C}$ & -2.14322400 & -0.79358300 & 1.42306100 \\
\hline
\end{tabular}




$\begin{array}{llll}\mathrm{H} & -1.96116300 & -1.87038800 & 1.47114600 \\ \mathrm{H} & -2.18202700 & -0.42576100 & 2.45154900 \\ \mathrm{H} & -3.11080600 & -0.62011800 & 0.94820500\end{array}$

INT2

\begin{tabular}{|c|c|c|c|}
\hline \multicolumn{3}{|c|}{ Zero-point correction= } & 0.619691 (Hartree/Particle) \\
\hline \multicolumn{3}{|c|}{ Thermal correction to Energy= } & 0.655945 \\
\hline \multicolumn{3}{|c|}{ Thermal correction to Enthalpy= } & 0.656889 \\
\hline \multicolumn{3}{|c|}{ Thermal correction to Gibbs Free Energy= } & 0.548294 \\
\hline \multicolumn{3}{|c|}{ Sum of electronic and zero-point Energies= } & -2239.009307 \\
\hline \multicolumn{3}{|c|}{ Sum of electronic and thermal Energies= } & -2238.973054 \\
\hline \multicolumn{3}{|c|}{ Sum of electronic and thermal Enthalpies= } & -2238.972110 \\
\hline \multicolumn{3}{|c|}{ Sum of electronic and thermal Free Energies= } & -2239.080705 \\
\hline \multicolumn{4}{|c|}{$\mathrm{E} / \mathrm{B} 2=-2240.085032$} \\
\hline \multicolumn{4}{|l|}{1} \\
\hline $\mathrm{C}$ & -0.84858500 & 0.74131000 & -0.45237900 \\
\hline $\mathrm{C}$ & -1.25605000 & 1.87723800 & 0.49882300 \\
\hline $\mathrm{C}$ & -1.15278100 & 1.28253300 & 1.90449100 \\
\hline $\mathrm{C}$ & -1.69964100 & -0.12858500 & 1.71268500 \\
\hline $\mathrm{N}$ & -1.25574600 & -0.52592000 & 0.32361500 \\
\hline $\mathrm{H}$ & -0.63504200 & 2.76233300 & 0.35730500 \\
\hline $\mathrm{H}$ & -1.74445200 & 1.83342200 & 2.63768700 \\
\hline $\mathrm{H}$ & -2.79038300 & -0.11195000 & 1.73512200 \\
\hline $\mathrm{H}$ & -0.11518200 & 1.26547700 & 2.24839600 \\
\hline $\mathrm{H}$ & -1.34088700 & -0.87954100 & 2.41246400 \\
\hline $\mathrm{H}$ & -2.29657100 & 2.14980600 & 0.29102900 \\
\hline $\mathrm{H}$ & -1.45842800 & 0.75759600 & -1.35692500 \\
\hline $\mathrm{C}$ & 0.59979900 & 0.65294100 & -0.89678400 \\
\hline $\mathrm{C}$ & 0.84036300 & 0.35487100 & -2.17761100 \\
\hline $\mathrm{H}$ & 0.02312300 & 0.17500500 & -2.87481600 \\
\hline $\mathrm{H}$ & 1.85053200 & 0.26175400 & -2.56470200 \\
\hline $\mathrm{P}$ & 1.92615400 & 0.85583800 & 0.38537900 \\
\hline $\mathrm{C}$ & 2.01731100 & 2.69216100 & 0.49733900 \\
\hline $\mathrm{C}$ & 2.40400200 & 3.23516300 & 1.72697900 \\
\hline $\mathrm{C}$ & 1.72276700 & 3.55739700 & -0.56455300 \\
\hline $\mathrm{C}$ & 2.50685700 & 4.61456700 & 1.89161600 \\
\hline $\mathrm{H}$ & 2.63021700 & 2.57250300 & 2.55888000 \\
\hline $\mathrm{C}$ & 1.81793800 & 4.93557500 & -0.39763500 \\
\hline $\mathrm{H}$ & 1.41273100 & 3.14731100 & -1.52286100 \\
\hline $\mathrm{C}$ & 2.21134000 & 5.46503400 & 0.83053200 \\
\hline $\mathrm{H}$ & 2.81470200 & 5.02337000 & 2.84838500 \\
\hline $\mathrm{H}$ & 1.58800200 & 5.59740800 & -1.22646500 \\
\hline $\mathrm{H}$ & 2.28615400 & 6.53986600 & 0.95886300 \\
\hline $\mathrm{C}$ & 3.42284100 & 0.39927400 & -0.57291300 \\
\hline
\end{tabular}




\begin{tabular}{|c|c|c|c|}
\hline C & 3.84726500 & -0.93039500 & -0.46992800 \\
\hline $\mathrm{C}$ & 4.13652400 & 1.28341800 & -1.38803300 \\
\hline $\mathrm{C}$ & 4.94935400 & -1.37753400 & -1.19165500 \\
\hline $\mathrm{H}$ & 3.32179100 & -1.61499600 & 0.19046100 \\
\hline $\mathrm{C}$ & 5.24604600 & 0.83601200 & -2.10047500 \\
\hline $\mathrm{H}$ & 3.83530600 & 2.32406500 & -1.45980700 \\
\hline $\mathrm{C}$ & 5.64945800 & -0.49406600 & -2.00895100 \\
\hline $\mathrm{H}$ & 5.26718200 & -2.41187400 & -1.10508300 \\
\hline $\mathrm{H}$ & 5.79903900 & 1.52898600 & -2.72614800 \\
\hline $\mathrm{H}$ & 6.51504700 & -0.83691700 & -2.56636700 \\
\hline$S$ & -2.52401300 & -1.49929900 & -0.55697700 \\
\hline $\mathrm{C}$ & -3.98688100 & -0.52778200 & -0.45852600 \\
\hline $\mathrm{C}$ & -4.90768600 & -0.77303400 & 0.56165600 \\
\hline $\mathrm{C}$ & -4.22944800 & 0.41445500 & -1.46044100 \\
\hline $\mathrm{C}$ & -6.07531500 & -0.02307500 & 0.59213000 \\
\hline $\mathrm{H}$ & -4.71889400 & -1.55399500 & 1.29208200 \\
\hline $\mathrm{C}$ & -5.40114300 & 1.15502800 & -1.40230600 \\
\hline $\mathrm{H}$ & -3.53653500 & 0.53060400 & -2.28720800 \\
\hline $\mathrm{C}$ & -6.33357200 & 0.95556700 & -0.37630300 \\
\hline $\mathrm{H}$ & -6.80743100 & -0.20772800 & 1.37200300 \\
\hline $\mathrm{H}$ & -5.60830200 & 1.88569600 & -2.17799000 \\
\hline $\mathrm{C}$ & -7.58934800 & 1.77952200 & -0.31643200 \\
\hline $\mathrm{H}$ & -7.92738400 & 2.05796000 & -1.31633400 \\
\hline $\mathrm{H}$ & -8.39431200 & 1.24122500 & 0.18670700 \\
\hline $\mathrm{H}$ & -7.40648700 & 2.70381400 & 0.24136100 \\
\hline $\mathrm{O}$ & -2.62681800 & -2.67849900 & 0.27103800 \\
\hline $\mathrm{O}$ & -2.06007700 & -1.50871800 & -1.92587300 \\
\hline $\mathrm{H}$ & -0.42278900 & -1.23731700 & 0.43592400 \\
\hline $\mathrm{C}$ & 2.89674700 & -4.25781500 & 1.14839100 \\
\hline $\mathrm{C}$ & 2.21546900 & -4.18912600 & -0.05654200 \\
\hline $\mathrm{C}$ & 1.17193000 & -3.27457100 & -0.20377200 \\
\hline $\mathrm{C}$ & 1.45915800 & -2.51793600 & 1.97199100 \\
\hline $\mathrm{C}$ & 2.51124700 & -3.40878200 & 2.17669200 \\
\hline $\mathrm{H}$ & 3.71183700 & -4.96082700 & 1.28645200 \\
\hline $\mathrm{H}$ & 2.47741800 & -4.83270400 & -0.88841000 \\
\hline $\mathrm{H}$ & 3.01286200 & -3.42945900 & 3.13737100 \\
\hline $\mathrm{N}$ & 0.80535600 & -2.44424900 & 0.79350200 \\
\hline $\mathrm{C}$ & 0.41214900 & -3.20844600 & -1.49320900 \\
\hline $\mathrm{H}$ & 0.91353000 & -3.78825200 & -2.26901500 \\
\hline $\mathrm{H}$ & -0.59509400 & -3.61447700 & -1.36239100 \\
\hline $\mathrm{H}$ & 0.31636300 & -2.17758300 & -1.83850000 \\
\hline $\mathrm{C}$ & 1.01548000 & -1.62807200 & 3.09770600 \\
\hline $\mathrm{H}$ & 0.07883800 & -1.99784800 & 3.52828300 \\
\hline $\mathrm{H}$ & 1.76025700 & -1.61570600 & 3.89465100 \\
\hline
\end{tabular}


TS2

$\begin{array}{lc}\text { Zero-point correction }= & 0.616734 \text { (Hartree/Particle) } \\ \text { Thermal correction to Energy= } & 0.652265 \\ \text { Thermal correction to Enthalpy= } & 0.653210 \\ \text { Thermal correction to Gibbs Free Energy= } & 0.547009 \\ \text { Sum of electronic and zero-point Energies }= & -2239.011797 \\ \text { Sum of electronic and thermal Energies= } & -2238.976266 \\ \text { Sum of electronic and thermal Enthalpies }= & -2238.975322 \\ \text { Sum of electronic and thermal Free Energies }= & -2239.081522\end{array}$

$\mathrm{E} / \mathrm{B} 2=-2240.083594$

\begin{tabular}{lrrr}
11 & & & \\
$\mathrm{C}$ & -0.76490300 & 0.72887200 & -0.52690000 \\
$\mathrm{C}$ & -1.16413600 & 1.91587700 & 0.36362200 \\
$\mathrm{C}$ & -1.10873100 & 1.37632500 & 1.79278400 \\
$\mathrm{C}$ & -1.69846400 & -0.02171700 & 1.63931700 \\
$\mathrm{~N}$ & -1.23448400 & -0.49459300 & 0.27969400 \\
$\mathrm{H}$ & -0.51935700 & 2.77974100 & 0.19982800 \\
$\mathrm{H}$ & -1.69889100 & 1.97345500 & 2.49026200 \\
$\mathrm{H}$ & -2.78780200 & 0.04210400 & 1.63929900 \\
$\mathrm{H}$ & -0.07994200 & 1.34357200 & 2.16162000 \\
$\mathrm{H}$ & -1.39408600 & -0.75260600 & 2.38423000 \\
$\mathrm{H}$ & -2.19368900 & 2.20206500 & 0.12184800 \\
$\mathrm{H}$ & -1.33911600 & 0.73371000 & -1.45480300 \\
$\mathrm{C}$ & 0.69586000 & 0.59780600 & -0.92285700 \\
$\mathrm{C}$ & 0.97378800 & 0.28166300 & -2.19179000 \\
$\mathrm{H}$ & 0.17635700 & 0.11162500 & -2.91402300 \\
$\mathrm{H}$ & 1.99410300 & 0.16638000 & -2.54486100 \\
$\mathrm{P}$ & 1.99001100 & 0.80158500 & 0.39150300 \\
$\mathrm{C}$ & 2.11064900 & 2.63679800 & 0.47940700 \\
$\mathrm{C}$ & 2.42751100 & 3.19603000 & 1.72138700 \\
$\mathrm{C}$ & 1.89740200 & 3.48588400 & -0.61441700 \\
$\mathrm{C}$ & 2.53869300 & 4.57685000 & 1.86894100 \\
$\mathrm{H}$ & 2.59086900 & 2.54483600 & 2.57687100 \\
$\mathrm{C}$ & 2.00235100 & 4.86521000 & -0.46551000 \\
$\mathrm{H}$ & 1.64165700 & 3.06150300 & -1.58257600 \\
$\mathrm{C}$ & 2.32370400 & 5.41129700 & 0.77649500 \\
$\mathrm{H}$ & 2.78898100 & 4.99935800 & 2.83651900 \\
$\mathrm{H}$ & 1.83495700 & 5.51565400 & -1.31803900 \\
$\mathrm{H}$ & 2.40464600 & 6.48727700 & 0.89099100 \\
$\mathrm{C}$ & 3.50265700 & 0.30029700 & -0.51775900 \\
$\mathrm{C}$ & 3.84914700 & -1.05373500 & -0.43953000 \\
$\mathrm{C}$ & 4.30044000 & 1.16669400 & -1.27071300 \\
& & & \\
\hline & & & \\
$\mathrm{H}$ & & &
\end{tabular}




\begin{tabular}{|c|c|c|c|}
\hline C & 4.95658200 & -1.54137100 & -1.12449400 \\
\hline $\mathrm{H}$ & 3.25419000 & -1.72543500 & 0.17382500 \\
\hline $\mathrm{C}$ & 5.41692600 & 0.67849600 & -1.94558100 \\
\hline $\mathrm{H}$ & 4.05953400 & 2.22390500 & -1.32355400 \\
\hline $\mathrm{C}$ & 5.74221600 & -0.67390300 & -1.87980200 \\
\hline $\mathrm{H}$ & 5.21265200 & -2.59424000 & -1.05862900 \\
\hline $\mathrm{H}$ & 6.03504100 & 1.35743200 & -2.52388400 \\
\hline $\mathrm{H}$ & 6.61286400 & -1.04846400 & -2.40814700 \\
\hline S & -2.52922300 & -1.41671000 & -0.57445100 \\
\hline $\mathrm{C}$ & -3.99484500 & -0.44593300 & -0.45086700 \\
\hline $\mathrm{C}$ & -4.91819500 & -0.72075200 & 0.55882700 \\
\hline $\mathrm{C}$ & -4.23142600 & 0.52914500 & -1.42087400 \\
\hline $\mathrm{C}$ & -6.08688800 & 0.02633900 & 0.60724900 \\
\hline $\mathrm{H}$ & -4.72529600 & -1.51711900 & 1.27126100 \\
\hline $\mathrm{C}$ & -5.40424300 & 1.26767100 & -1.34505300 \\
\hline $\mathrm{H}$ & -3.53037700 & 0.67816300 & -2.23548800 \\
\hline $\mathrm{C}$ & -6.34420100 & 1.03066200 & -0.33491100 \\
\hline $\mathrm{H}$ & -6.81896300 & -0.17683200 & 1.38284700 \\
\hline $\mathrm{H}$ & -5.60407700 & 2.02859400 & -2.09310900 \\
\hline $\mathrm{C}$ & -7.63023800 & 1.80782100 & -0.28832000 \\
\hline $\mathrm{H}$ & -8.41639300 & 1.26418100 & -0.82196800 \\
\hline $\mathrm{H}$ & -7.96891100 & 1.95263700 & 0.73954300 \\
\hline $\mathrm{H}$ & -7.52119500 & 2.78527600 & -0.76143300 \\
\hline $\mathrm{O}$ & -2.63800500 & -2.60831400 & 0.24000600 \\
\hline $\mathrm{O}$ & -2.11227100 & -1.42919100 & -1.96070000 \\
\hline $\mathrm{H}$ & -0.37131800 & -1.31443700 & 0.46571200 \\
\hline $\mathrm{C}$ & 2.62841600 & -4.29569000 & 1.20500400 \\
\hline $\mathrm{C}$ & 1.97087800 & -4.21152900 & -0.01091100 \\
\hline $\mathrm{C}$ & 0.98404400 & -3.24366500 & -0.19779600 \\
\hline $\mathrm{C}$ & 1.28431600 & -2.45757700 & 1.97726600 \\
\hline $\mathrm{C}$ & 2.27884900 & -3.40413900 & 2.20929700 \\
\hline $\mathrm{H}$ & 3.40000500 & -5.04078800 & 1.36898800 \\
\hline $\mathrm{H}$ & 2.20706900 & -4.88493400 & -0.82647900 \\
\hline $\mathrm{H}$ & 2.76566000 & -3.43253700 & 3.17711100 \\
\hline $\mathrm{N}$ & 0.65275600 & -2.37485700 & 0.78299900 \\
\hline $\mathrm{C}$ & 0.25736200 & -3.17254400 & -1.50403600 \\
\hline $\mathrm{H}$ & 0.78324200 & -3.75317600 & -2.26252200 \\
\hline $\mathrm{H}$ & -0.75147400 & -3.58191300 & -1.39992200 \\
\hline $\mathrm{H}$ & 0.16737200 & -2.14444300 & -1.85430400 \\
\hline $\mathrm{C}$ & 0.88772400 & -1.52962400 & 3.08821600 \\
\hline $\mathrm{H}$ & -0.04951400 & -1.86230100 & 3.54627100 \\
\hline $\mathrm{H}$ & 1.65104400 & -1.52926200 & 3.86726100 \\
\hline $\mathrm{H}$ & 0.78240400 & -0.50855700 & 2.72069300 \\
\hline
\end{tabular}


$\mathrm{P}$

$\begin{array}{lc}\text { Zero-point correction }= & 0.620449 \text { (Hartree/Particle) } \\ \text { Thermal correction to Energy= } & 0.657122 \\ \text { Thermal correction to Enthalpy= } & 0.658067 \\ \text { Thermal correction to Gibbs Free Energy= } & 0.548915 \\ \text { Sum of electronic and zero-point Energies= } & -2239.044364 \\ \text { Sum of electronic and thermal Energies= } & -2239.007691 \\ \text { Sum of electronic and thermal Enthalpies= } & -2239.006747 \\ \text { Sum of electronic and thermal Free Energies }= & -2239.115898\end{array}$

$\mathrm{E} / \mathrm{B} 2=-2240.119478$

11

$\begin{array}{llll}\text { C } & -1.04232900 & 1.76267600 & -0.63512000\end{array}$

$\begin{array}{llll}\text { C } & -0.81828100 & 3.18415100 & -0.04111300\end{array}$

$\begin{array}{llll}\mathrm{C} & -0.87923400 & 3.01732900 & 1.48334700\end{array}$

$\begin{array}{llll}\mathrm{C} & -1.84987400 & 1.85472300 & 1.66369100\end{array}$

$\begin{array}{llll}\mathrm{N} & -1.42331800 & 0.97523500 & 0.56471200\end{array}$

$\begin{array}{llll}\mathrm{H} & 0.13080500 & 3.60416200 & -0.38077200\end{array}$

$\begin{array}{llll}\mathrm{H} & -1.21061500 & 3.92187100 & 1.99545800\end{array}$

$\begin{array}{llll}\mathrm{H} & -2.89303900 & 2.18008100 & 1.53298300\end{array}$

$\begin{array}{llll}\mathrm{H} & 0.10463400 & 2.73734100 & 1.87559600\end{array}$

$\begin{array}{llll}\mathrm{H} & -1.75661000 & 1.33112700 & 2.61725000\end{array}$

$\mathrm{H} \quad-1.61963000 \quad 3.84436000 \quad-0.38199400$

$\begin{array}{llll}\mathrm{H} & -1.87447900 & 1.79176200 & -1.35340200\end{array}$

$\begin{array}{llll}\mathrm{C} & 0.17516800 & 1.18999800 & -1.33340000\end{array}$

$\begin{array}{llll}\mathrm{C} & 0.22857900 & 1.15141700 & -2.66422200\end{array}$

$\begin{array}{llll}\mathrm{H} & -0.61075400 & 1.48929700 & -3.26975000\end{array}$

$\begin{array}{llll}\mathrm{H} & 1.09336300 & 0.76675700 & -3.19647200\end{array}$

$\begin{array}{llll}\mathrm{P} & 1.50205800 & 0.57342900 & -0.20268200\end{array}$

$\begin{array}{llll}\text { C } & 2.66077300 & 2.00336500 & -0.13445600\end{array}$

$\begin{array}{llll}\mathrm{C} & 3.19414100 & 2.33129100 & 1.11574400\end{array}$

$\begin{array}{llll}\text { C } & 3.03831200 & 2.75616800 & -1.25313300\end{array}$

$\begin{array}{llll}\mathrm{C} & 4.09253400 & 3.38834200 & 1.25178200\end{array}$

$\begin{array}{llll}\mathrm{H} & 2.89971900 & 1.75210500 & 1.98894400\end{array}$

$\begin{array}{llll}\mathrm{C} & 3.93244000 & 3.81218300 & -1.11876000\end{array}$

$\begin{array}{llll}\mathrm{H} & 2.62407000 & 2.51630800 & -2.22860800\end{array}$

$\begin{array}{llll}\mathrm{C} & 4.46049700 & 4.12865600 & 0.13325000\end{array}$

$\begin{array}{llll}\mathrm{H} & 4.50003000 & 3.63421100 & 2.22701000\end{array}$

$\begin{array}{llll}\mathrm{H} & 4.21970800 & 4.39120600 & -1.99041600\end{array}$

$\begin{array}{llll}\mathrm{H} & 5.15779200 & 4.95385900 & 0.23405700\end{array}$

$\begin{array}{llll}\text { C } & 2.39864700 & -0.64346300 & -1.24862000\end{array}$

$\begin{array}{llll}\mathrm{C} & 1.68088700 & -1.62191700 & -1.95167300\end{array}$

$\begin{array}{llll}\text { C } & 3.79146800 & -0.74838200 & -1.17418000\end{array}$

$\begin{array}{llll}\mathrm{C} & 2.34770200 & -2.67384700 & -2.57434900\end{array}$

$\mathrm{H} \quad 0.59713000 \quad-1.55273700 \quad-2.00945400$ 


\begin{tabular}{|c|c|c|c|}
\hline $\mathrm{C}$ & 4.45455800 & -1.80942600 & -1.78952000 \\
\hline $\mathrm{H}$ & 4.36203200 & 0.00524600 & -0.63684800 \\
\hline $\mathrm{C}$ & 3.73623000 & -2.77438600 & -2.49131100 \\
\hline $\mathrm{H}$ & 1.78165600 & -3.41300200 & -3.13398100 \\
\hline $\mathrm{H}$ & 5.53706300 & -1.87154200 & -1.73397500 \\
\hline $\mathrm{H}$ & 4.25532300 & -3.59069800 & -2.98317700 \\
\hline S & -2.10655100 & -0.50777000 & 0.39335800 \\
\hline $\mathrm{C}$ & -3.80323600 & -0.28472400 & -0.03734300 \\
\hline $\mathrm{C}$ & -4.75287100 & -0.12375300 & 0.96982600 \\
\hline $\mathrm{C}$ & -4.14402700 & -0.20670600 & -1.38686000 \\
\hline $\mathrm{C}$ & -6.07216500 & 0.11611000 & 0.60663800 \\
\hline $\mathrm{H}$ & -4.46263300 & -0.21104100 & 2.01213600 \\
\hline $\mathrm{C}$ & -5.46951000 & 0.03322500 & -1.72478700 \\
\hline $\mathrm{H}$ & -3.38515300 & -0.35927600 & -2.14783800 \\
\hline $\mathrm{C}$ & -6.44902400 & 0.19684000 & -0.73840300 \\
\hline $\mathrm{H}$ & -6.82582200 & 0.23583500 & 1.37901000 \\
\hline $\mathrm{H}$ & -5.75326600 & 0.08653500 & -2.77168400 \\
\hline $\mathrm{C}$ & -7.88744800 & 0.41840300 & -1.12039400 \\
\hline $\mathrm{H}$ & -8.38858700 & -0.54153200 & -1.28072800 \\
\hline $\mathrm{H}$ & -8.42953500 & 0.94926100 & -0.33562300 \\
\hline $\mathrm{H}$ & -7.96695700 & 0.99132200 & -2.04659800 \\
\hline $\mathrm{O}$ & -2.04723300 & -1.14048700 & 1.72078500 \\
\hline $\mathrm{O}$ & -1.42146300 & -1.16622200 & -0.71386400 \\
\hline $\mathrm{H}$ & -0.42277100 & -2.11019300 & 1.73189200 \\
\hline $\mathrm{C}$ & 3.26815900 & -2.64542900 & 1.59024500 \\
\hline $\mathrm{C}$ & 2.43284000 & -3.42301000 & 0.79548200 \\
\hline $\mathrm{C}$ & 1.06118500 & -3.23527100 & 0.86143200 \\
\hline $\mathrm{C}$ & 1.35942200 & -1.51773800 & 2.50476700 \\
\hline $\mathrm{C}$ & 2.73264300 & -1.69620000 & 2.45657500 \\
\hline $\mathrm{H}$ & 4.34355400 & -2.77634300 & 1.52765200 \\
\hline $\mathrm{H}$ & 2.83384000 & -4.14646500 & 0.09672200 \\
\hline $\mathrm{H}$ & 3.36763300 & -1.07819000 & 3.07981500 \\
\hline $\mathrm{N}$ & 0.59095800 & -2.30024200 & 1.71741500 \\
\hline $\mathrm{C}$ & 0.06139100 & -3.99395100 & 0.05103300 \\
\hline $\mathrm{H}$ & 0.57385100 & -4.63440000 & -0.66648300 \\
\hline $\mathrm{H}$ & -0.55905600 & -4.62008900 & 0.69956000 \\
\hline $\mathrm{H}$ & -0.59103000 & -3.29812200 & -0.48524200 \\
\hline $\mathrm{C}$ & 0.66697100 & -0.46932200 & 3.31177700 \\
\hline $\mathrm{H}$ & -0.25666200 & -0.84802800 & 3.75461600 \\
\hline $\mathrm{H}$ & 1.32470900 & -0.09658000 & 4.09715200 \\
\hline $\mathrm{H}$ & 0.41042800 & 0.35309000 & 2.63177900 \\
\hline
\end{tabular}

$\mathrm{TS} 1^{\text {anti }}$

Zero-point correction=

0.551085 (Hartree/Particle) S13 


$\begin{array}{lc}\text { Thermal correction to Energy= } & 0.583548 \\ \text { Thermal correction to Enthalpy= } & 0.584493 \\ \text { Thermal correction to Gibbs Free Energy= } & 0.485597 \\ \text { Sum of electronic and zero-point Energies= } & -2143.183249 \\ \text { Sum of electronic and thermal Energies= } & -2143.150786 \\ \text { Sum of electronic and thermal Enthalpies= } & -2143.149842 \\ \text { Sum of electronic and thermal Free Energies }= & -2143.248737\end{array}$

$\mathrm{E} / \mathrm{B} 2=-2144.175973$

11

$\begin{array}{lrrr}\mathrm{C} & 0.75007600 & 0.75405700 & 0.15584600 \\ \mathrm{C} & 3.48341600 & 0.39450900 & -0.57333300 \\ \mathrm{~N} & 2.40450000 & -0.47359000 & -1.11037600 \\ \mathrm{H} & 0.90883300 & 1.27847000 & -0.78199400 \\ \mathrm{C} & -0.54938800 & 0.19288100 & 0.35000500 \\ \mathrm{C} & -0.76061200 & -0.66671500 & 1.38122800 \\ \mathrm{H} & -1.74765300 & -1.08248300 & 1.57035300 \\ \mathrm{H} & 0.05593800 & -0.99764000 & 2.01645600 \\ \mathrm{P} & -1.81453600 & 0.58402400 & -0.95711600 \\ \mathrm{C} & -1.69220800 & 2.41970200 & -0.94329600 \\ \mathrm{C} & -2.07129500 & 3.07391000 & -2.12130000 \\ \mathrm{C} & -1.24185500 & 3.18674100 & 0.14037300 \\ \mathrm{C} & -2.01844700 & 4.46204300 & -2.20946100 \\ \mathrm{H} & -2.40582700 & 2.49187300 & -2.97597000 \\ \mathrm{C} & -1.17692200 & 4.57409900 & 0.04749100 \\ \mathrm{H} & -0.95149900 & 2.70507300 & 1.07158400 \\ \mathrm{C} & -1.56840500 & 5.21270400 & -1.12674200 \\ \mathrm{H} & -2.32011700 & 4.95636900 & -3.12684700 \\ \mathrm{H} & -0.82927800 & 5.15676300 & 0.89433600 \\ \mathrm{H} & -1.52104200 & 6.29422400 & -1.19754100 \\ \mathrm{C} & -3.34181500 & 0.22483400 & -0.00340300 \\ \mathrm{C} & -4.02689200 & -0.95651200 & -0.30612900 \\ \mathrm{C} & -3.81763600 & 1.04936300 & 1.02249000 \\ \mathrm{C} & -5.16521800 & -1.31518500 & 0.41391300 \\ \mathrm{H} & -3.67339200 & -1.59098000 & -1.11507200 \\ \mathrm{C} & -4.95637900 & 0.69230400 & 1.73660500 \\ \mathrm{H} & -3.30989200 & 1.98204000 & 1.24968400 \\ \mathrm{C} & -5.62868500 & -0.49133200 & 1.43561000 \\ \mathrm{H} & -5.69607500 & -2.22914000 & 0.16659800 \\ \mathrm{H} & -5.32534100 & 1.34177500 & 2.52351200 \\ \mathrm{H} & -6.51961900 & -0.76459900 & 1.99112900 \\ \mathrm{~S} & 2.65656800 & -2.15408200 & -0.82159600 \\ \mathrm{C} & 1.00842500 & -2.75267800 & -0.59582500 \\ \mathrm{C} & 0.67979400 & -3.34100700 & 0.62191700 \\ \mathrm{C} & & -2.65923800 & -1.63623200\end{array}$




\begin{tabular}{lrrr}
$\mathrm{C}$ & -0.61141900 & -3.82647000 & 0.79872000 \\
$\mathrm{H}$ & 1.43016400 & -3.41601800 & 1.40272200 \\
$\mathrm{C}$ & -1.19849600 & -3.14039500 & -1.43463900 \\
$\mathrm{H}$ & 0.37288000 & -2.23201500 & -2.59407400 \\
$\mathrm{C}$ & -1.56796000 & -3.72837300 & -0.22024000 \\
$\mathrm{H}$ & -0.88080000 & -4.30148100 & 1.73826400 \\
$\mathrm{H}$ & -1.92189300 & -3.07557200 & -2.24237700 \\
$\mathrm{C}$ & -2.95736500 & -4.26487500 & -0.00330300 \\
$\mathrm{H}$ & -2.92250000 & -5.31612700 & 0.29497000 \\
$\mathrm{H}$ & -3.46669700 & -3.71156900 & 0.79235600 \\
$\mathrm{H}$ & -3.56075500 & -4.18981500 & -0.91052900 \\
$\mathrm{O}$ & 3.35604100 & -2.21900500 & 0.44906900 \\
$\mathrm{O}$ & 3.20993600 & -2.70520000 & -2.04322600 \\
$\mathrm{H}$ & 2.31105100 & -0.35894700 & -2.12152300 \\
$\mathrm{C}$ & 4.07496300 & 0.95508000 & 1.82356400 \\
$\mathrm{C}$ & 3.70416700 & 1.27630200 & 3.13238500 \\
$\mathrm{C}$ & 2.36209800 & 1.40889600 & 3.47730400 \\
$\mathrm{C}$ & 1.38332600 & 1.20995500 & 2.51330200 \\
$\mathrm{C}$ & 1.74782800 & 0.91121700 & 1.1857100 \\
$\mathrm{C}$ & 3.11390800 & 0.78853000 & 0.84161700 \\
$\mathrm{H}$ & 5.12203000 & 0.80406400 & 1.57855900 \\
$\mathrm{H}$ & 4.47139100 & 1.40609200 & 3.88838300 \\
$\mathrm{H}$ & 2.07958100 & 1.66279800 & 4.49253600 \\
$\mathrm{H}$ & 0.33486200 & 1.34506000 & 2.76010600 \\
$\mathrm{H}$ & 4.43494400 & -0.15012600 & -0.53955000 \\
$\mathrm{C}$ & 3.66003900 & 1.61199100 & -1.49956200 \\
$\mathrm{C}$ & 4.79653900 & 2.52705400 & -1.05364600 \\
$\mathrm{H}$ & 2.72481300 & 2.18282200 & -1.55544800 \\
$\mathrm{H}$ & 3.86949800 & 1.23457600 & -2.50728600 \\
$\mathrm{H}$ & 4.94333500 & 3.32618200 & -1.78202800 \\
$\mathrm{H}$ & 4.58578200 & 2.98894000 & -0.08608400 \\
$\mathrm{H}$ & 5.73636700 & 1.97275400 & -0.97289300 \\
& & & \\
\hline
\end{tabular}

$\mathrm{TS}^{\mathrm{syn}}$

$\begin{array}{lc}\text { Zero-point correction }= & 0.551105 \text { (Hartree/Particle) } \\ \text { Thermal correction to Energy= } & 0.583501 \\ \text { Thermal correction to Enthalpy= } & 0.584445 \\ \text { Thermal correction to Gibbs Free Energy= } & 0.486184 \\ \text { Sum of electronic and zero-point Energies }= & -2143.180516 \\ \text { Sum of electronic and thermal Energies }= & -2143.148120 \\ \text { Sum of electronic and thermal Enthalpies= } & -2143.147176 \\ \text { Sum of electronic and thermal Free Energies }= & -2143.245437 \\ \text { E/B2=-2144.173146 } & \\ 11 & \end{array}$




\begin{tabular}{|c|c|c|c|}
\hline $\mathrm{C}$ & 0.64999000 & 0.97389300 & 0.11588600 \\
\hline $\mathrm{C}$ & 3.27739300 & 0.84600600 & -0.81980000 \\
\hline $\mathrm{N}$ & 2.31451600 & -0.22768300 & -1.19531800 \\
\hline $\mathrm{H}$ & 0.75029600 & 1.47504500 & -0.84511700 \\
\hline $\mathrm{C}$ & -0.60119000 & 0.32452100 & 0.35399000 \\
\hline $\mathrm{C}$ & -0.73209200 & -0.52090500 & 1.40926200 \\
\hline $\mathrm{H}$ & -1.68171600 & -1.00696700 & 1.61972100 \\
\hline $\mathrm{H}$ & 0.11422800 & -0.77044800 & 2.04189100 \\
\hline$P$ & -1.87572100 & 0.56998000 & -0.97202500 \\
\hline $\mathrm{C}$ & -2.05981100 & 2.39715700 & -0.85913100 \\
\hline $\mathrm{C}$ & -2.56567700 & 3.04199200 & -1.99412600 \\
\hline $\mathrm{C}$ & -1.72636300 & 3.16605000 & 0.26404100 \\
\hline $\mathrm{C}$ & -2.74975000 & 4.42101600 & -2.00133000 \\
\hline $\mathrm{H}$ & -2.81520500 & 2.46035800 & -2.87762300 \\
\hline $\mathrm{C}$ & -1.89826100 & 4.54767800 & 0.25119500 \\
\hline $\mathrm{H}$ & -1.34035400 & 2.68913800 & 1.16196500 \\
\hline C & -2.41273100 & 5.17546500 & -0.87999500 \\
\hline $\mathrm{H}$ & -3.14816800 & 4.90728500 & -2.88545500 \\
\hline $\mathrm{H}$ & -1.63614700 & 5.13270800 & 1.12661500 \\
\hline $\mathrm{H}$ & -2.54928200 & 6.25161900 & -0.88831600 \\
\hline $\mathrm{C}$ & -3.37243700 & -0.08302000 & -0.13554400 \\
\hline $\mathrm{C}$ & -3.96301200 & -1.22537100 & -0.68281200 \\
\hline $\mathrm{C}$ & -3.93533600 & 0.50051500 & 1.00666800 \\
\hline $\mathrm{C}$ & -5.09492300 & -1.78595000 & -0.09361200 \\
\hline $\mathrm{H}$ & -3.54522500 & -1.66506000 & -1.58413200 \\
\hline $\mathrm{C}$ & -5.06118800 & -0.06303500 & 1.59707100 \\
\hline $\mathrm{H}$ & -3.50285000 & 1.40703700 & 1.42226500 \\
\hline $\mathrm{C}$ & -5.64006500 & -1.20771300 & 1.04832800 \\
\hline $\mathrm{H}$ & -5.55328800 & -2.66701900 & -0.53120600 \\
\hline $\mathrm{H}$ & -5.49692600 & 0.39622200 & 2.47822100 \\
\hline $\mathrm{H}$ & -6.52431800 & -1.63921000 & 1.50562600 \\
\hline S & 2.76093400 & -1.82782300 & -0.75067000 \\
\hline $\mathrm{C}$ & 1.18221200 & -2.56527000 & -0.44454500 \\
\hline $\mathrm{C}$ & 0.93655600 & -3.10320600 & 0.81459000 \\
\hline $\mathrm{C}$ & 0.23456700 & -2.62176700 & -1.46175800 \\
\hline $\mathrm{C}$ & -0.30138700 & -3.68940800 & 1.05945000 \\
\hline $\mathrm{H}$ & 1.70730800 & -3.05880400 & 1.57778000 \\
\hline $\mathrm{C}$ & -0.99962900 & -3.20098400 & -1.19249100 \\
\hline $\mathrm{H}$ & 0.45278200 & -2.23534400 & -2.45299200 \\
\hline $\mathrm{C}$ & -1.28869300 & -3.73669100 & 0.06784500 \\
\hline $\mathrm{H}$ & -0.50288800 & -4.12511800 & 2.03429000 \\
\hline $\mathrm{H}$ & -1.74164400 & -3.26267300 & -1.98284700 \\
\hline $\mathrm{C}$ & -2.62803900 & -4.35634400 & 0.36065500 \\
\hline $\mathrm{H}$ & -2.50986000 & -5.32653300 & 0.84951800 \\
\hline
\end{tabular}




$\begin{array}{lrrr}\mathrm{H} & -3.21339600 & -3.71429500 & 1.02757900 \\ \mathrm{H} & -3.20698200 & -4.50085900 & -0.55390400 \\ \mathrm{O} & 3.46858800 & -1.70179400 & 0.51179300 \\ \mathrm{O} & 3.36376400 & -2.43873400 & -1.92064200 \\ \mathrm{H} & 2.16445200 & -0.23510200 & -2.20584700 \\ \mathrm{C} & 4.73701700 & 0.58267000 & -1.21535100 \\ \mathrm{C} & 5.51984400 & 1.87837300 & -1.43082200 \\ \mathrm{H} & 4.73342100 & 0.00660400 & -2.14655400 \\ \mathrm{H} & 5.20780500 & -0.05989600 & -0.46503700 \\ \mathrm{H} & 6.56223500 & 1.65882700 & -1.66783300 \\ \mathrm{H} & 5.10218900 & 2.44800300 & -2.26624800 \\ \mathrm{H} & 5.50634200 & 2.52843400 & -0.55171100 \\ \mathrm{H} & 2.93101700 & 1.70775000 & -1.41317100 \\ \mathrm{C} & 4.03864300 & 1.40575900 & 1.56523900 \\ \mathrm{C} & 3.72887800 & 1.67043500 & 2.90371700 \\ \mathrm{C} & 2.40838400 & 1.70541700 & 3.34152700 \\ \mathrm{C} & 1.38455800 & 1.45612000 & 2.43941900 \\ \mathrm{C} & 1.68352800 & 1.20611400 & 1.08627800 \\ \mathrm{C} & 3.03183800 & 1.19212600 & 0.64169400 \\ \mathrm{H} & 5.07803600 & 1.34040200 & 1.26535800 \\ \mathrm{H} & 4.53445000 & 1.84010600 & 3.61052200 \\ \mathrm{H} & 2.17944400 & 1.92538600 & 4.37789200 \\ \mathrm{H} & 0.34775000 & 1.51603000 & 2.75464000\end{array}$

\section{References}

(1) (a) Zhao, Y.; Truhlar, D. G., Comparative DFT Study of van der Waals Complexes: Rare-Gas Dimers, Alkaline-Earth Dimers, Zinc Dimer, and Zinc-Rare-Gas Dimers. J. Phys. Chem. A 2006, 110, 5121-5129; (b) Zhao, Y.; Truhlar, D. G., The M06 suite of density functionals for main group thermochemistry, thermochemical kinetics, noncovalent interactions, excited states, and transition elements: two new functionals and systematic testing of four M06-class functionals and 12 other functionals. Theor. Chem. Acc. 2008, 120, 215-241. 


\section{Copies of NMR Spectra}
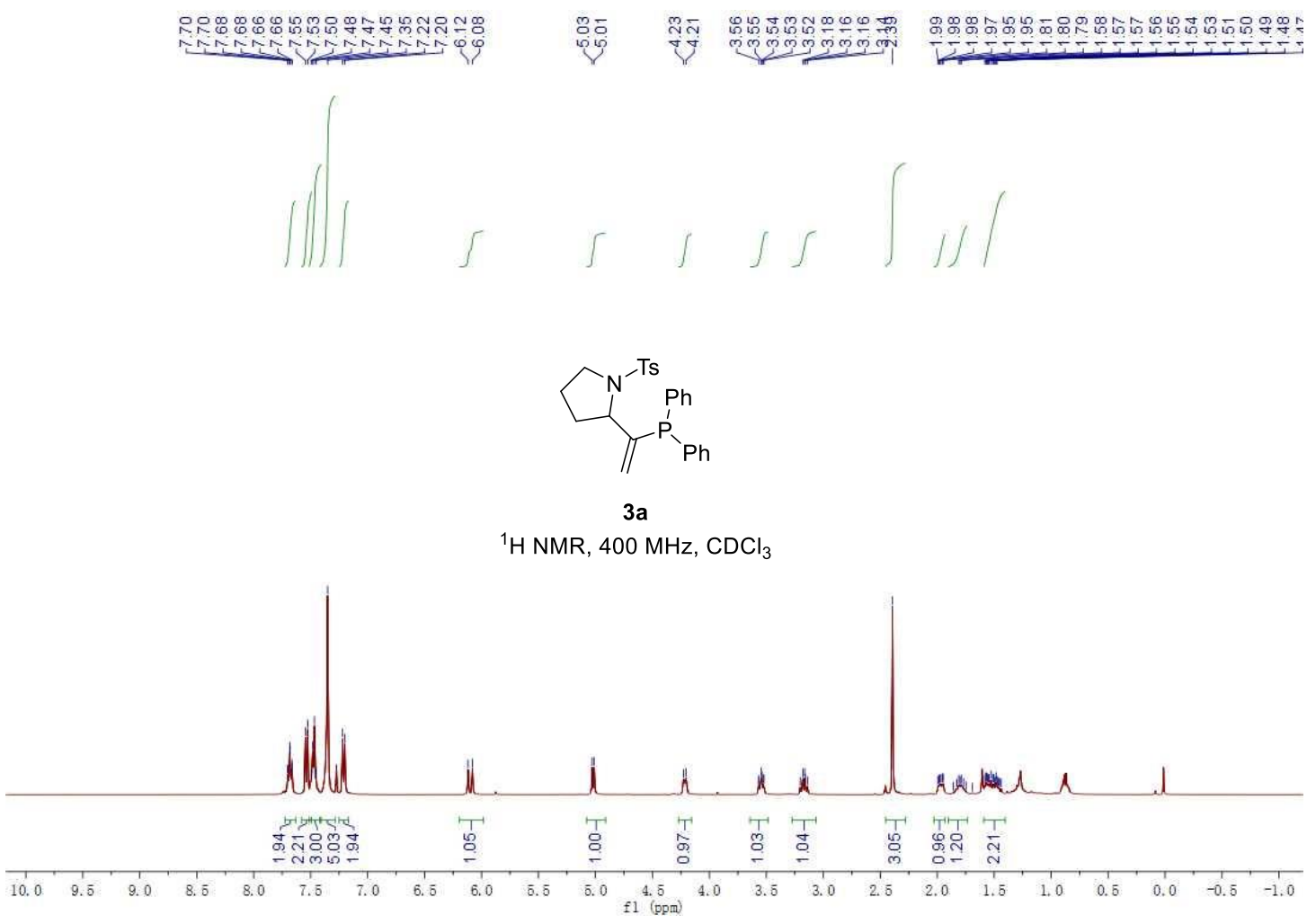

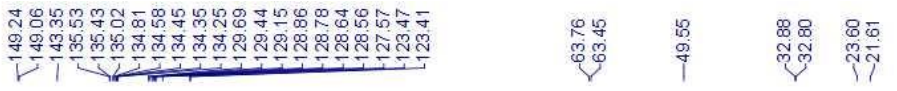

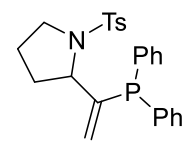

3a

${ }^{13} \mathrm{C}\left\{{ }^{1} \mathrm{H}\right\} \mathrm{NMR}, 100 \mathrm{MHz}, \mathrm{CDCl}_{3}$

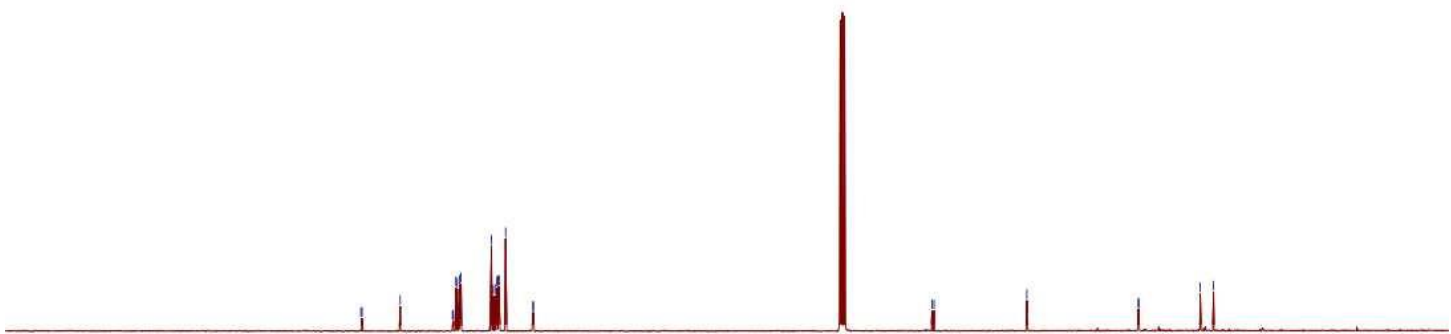

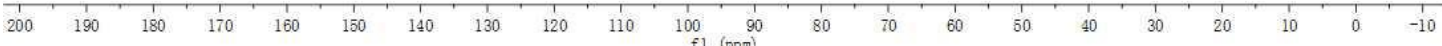




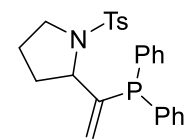

$3 a$

${ }^{31} \mathrm{P} \mathrm{NMR}, 162 \mathrm{MHz}, \mathrm{CDCl}_{3}$

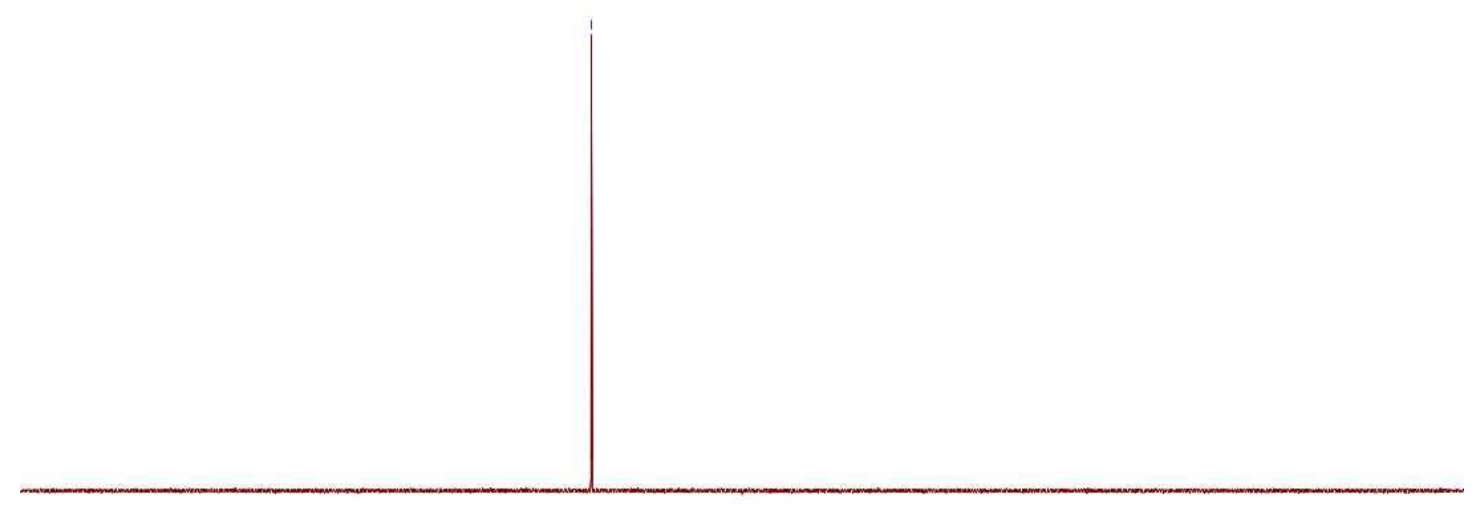

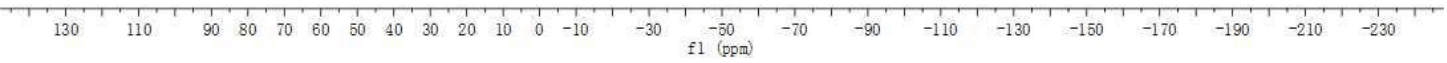

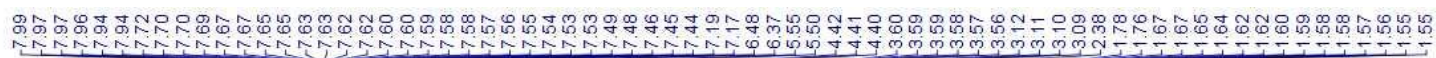
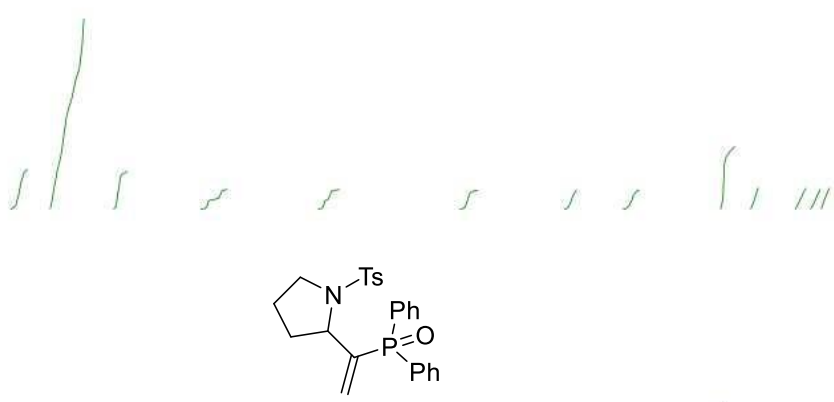

3a'

${ }^{1} \mathrm{H} \mathrm{NMR}, 400 \mathrm{MHz}, \mathrm{CDCl}_{3}$

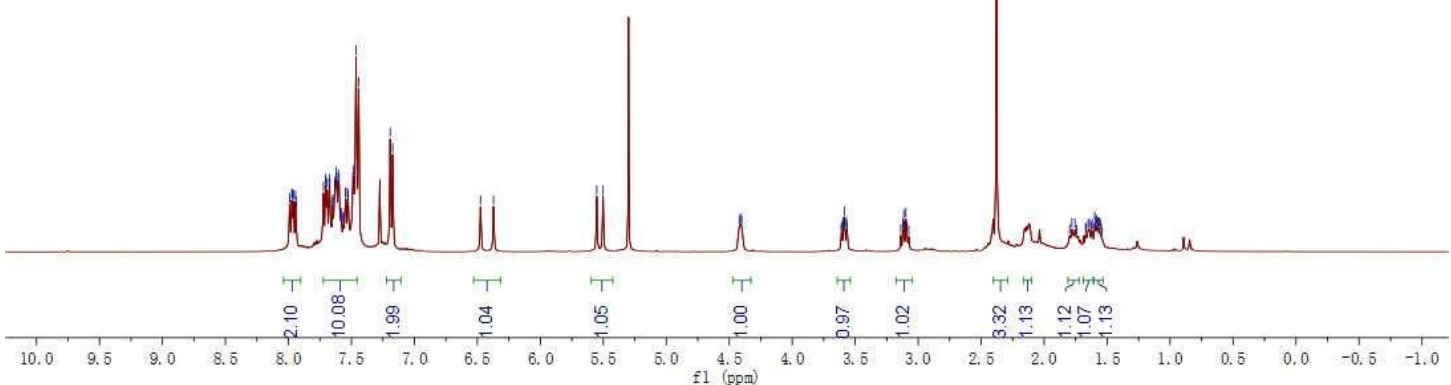




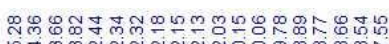

$3 a^{\prime}$

${ }^{13} \mathrm{C}\left\{{ }^{1} \mathrm{H}\right\} \mathrm{NMR}, 100 \mathrm{MHz}, \mathrm{CDCl}_{3}$
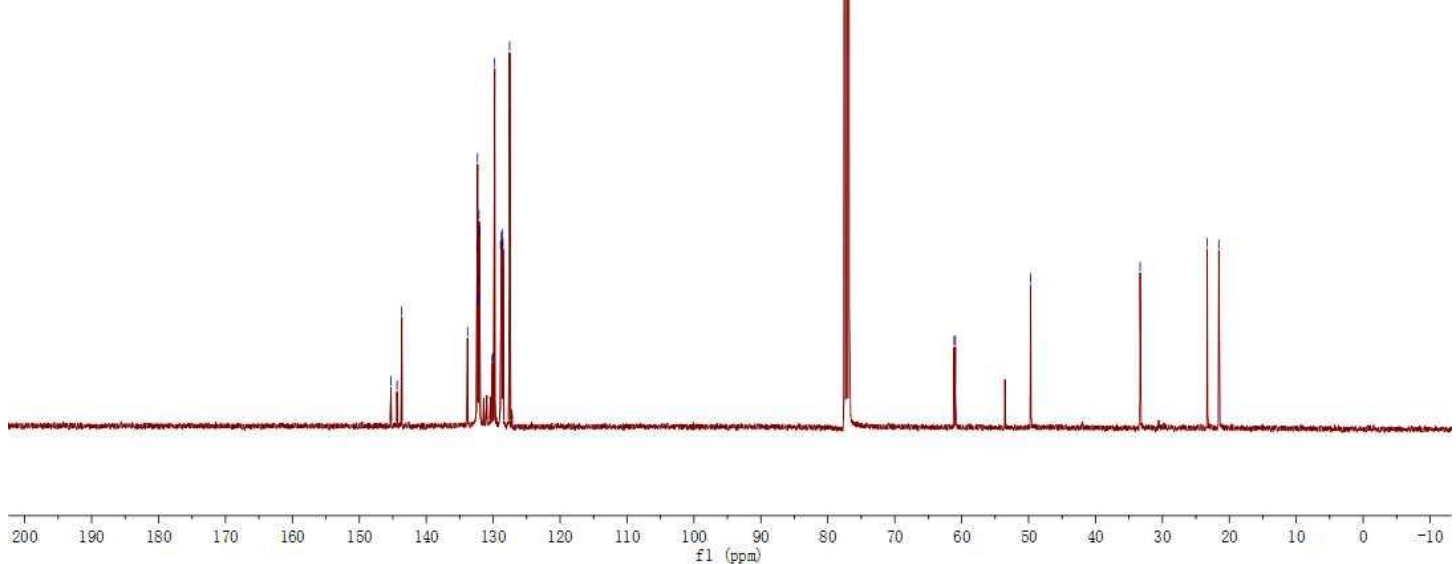

$\frac{\text { จี }}{\text { ฺ }}$

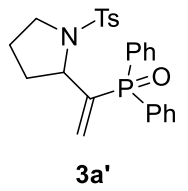

${ }^{31} \mathrm{P}$ NMR, $162 \mathrm{MHz}, \mathrm{CDCl}_{3}$

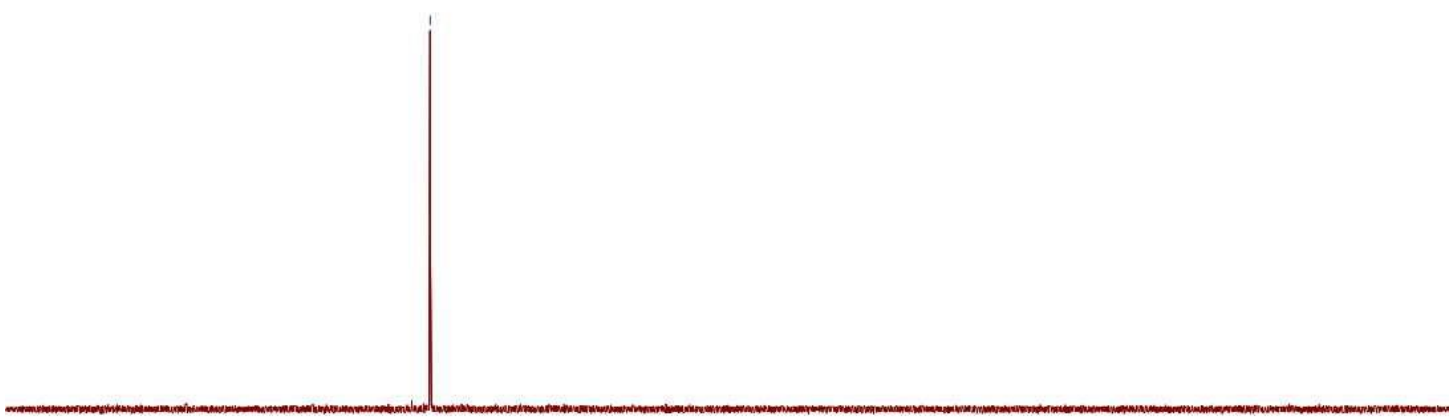

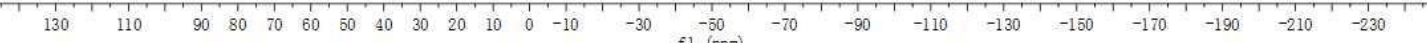




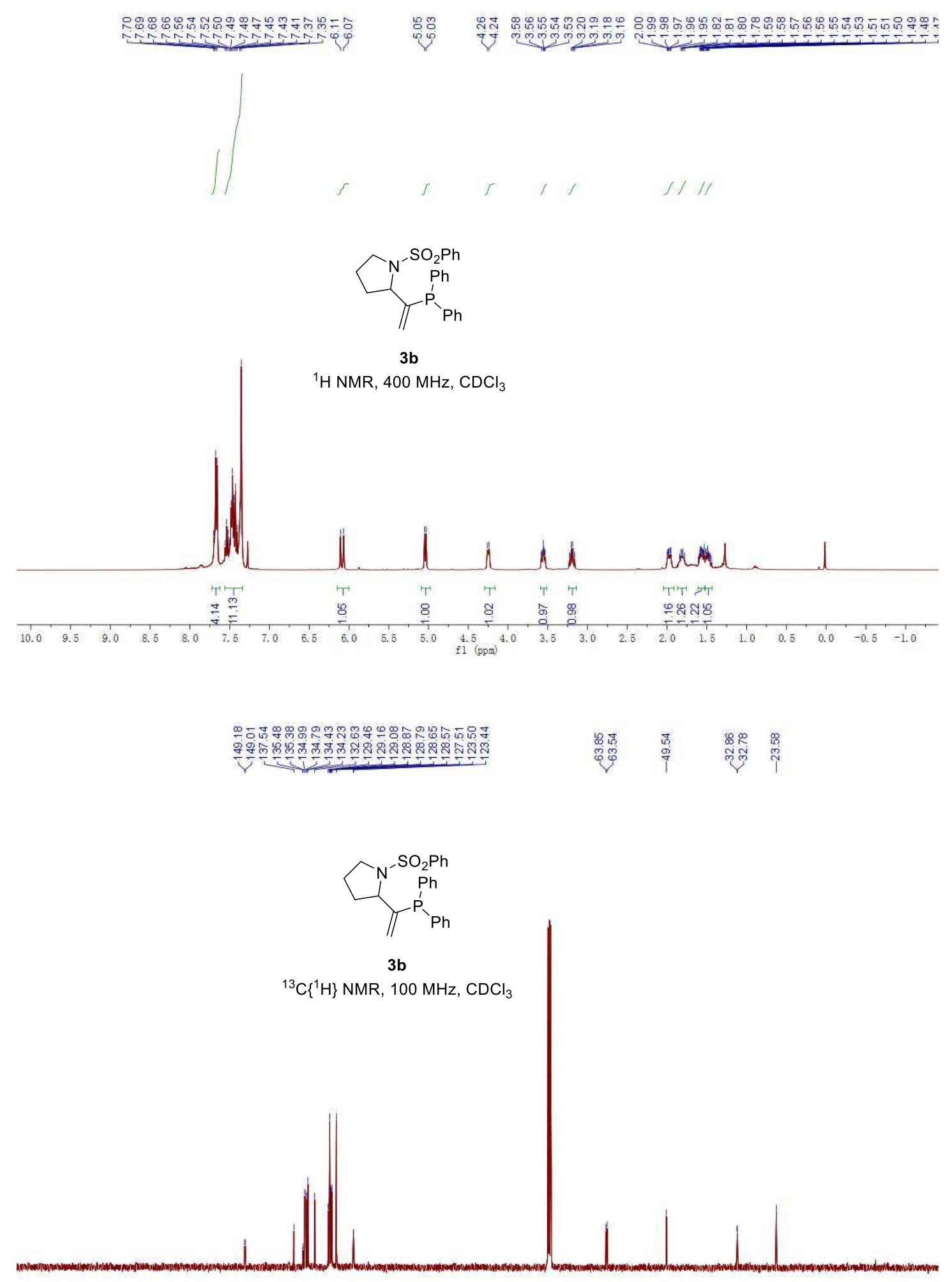

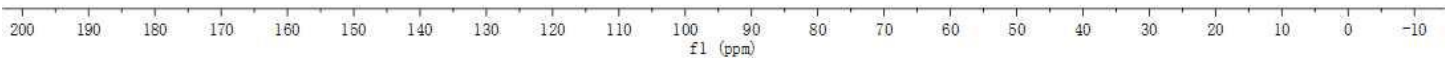




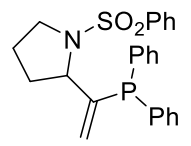

$3 \mathbf{b}$

${ }^{31} \mathrm{P} \mathrm{NMR}, 162 \mathrm{MHz}, \mathrm{CDCl}_{3}$

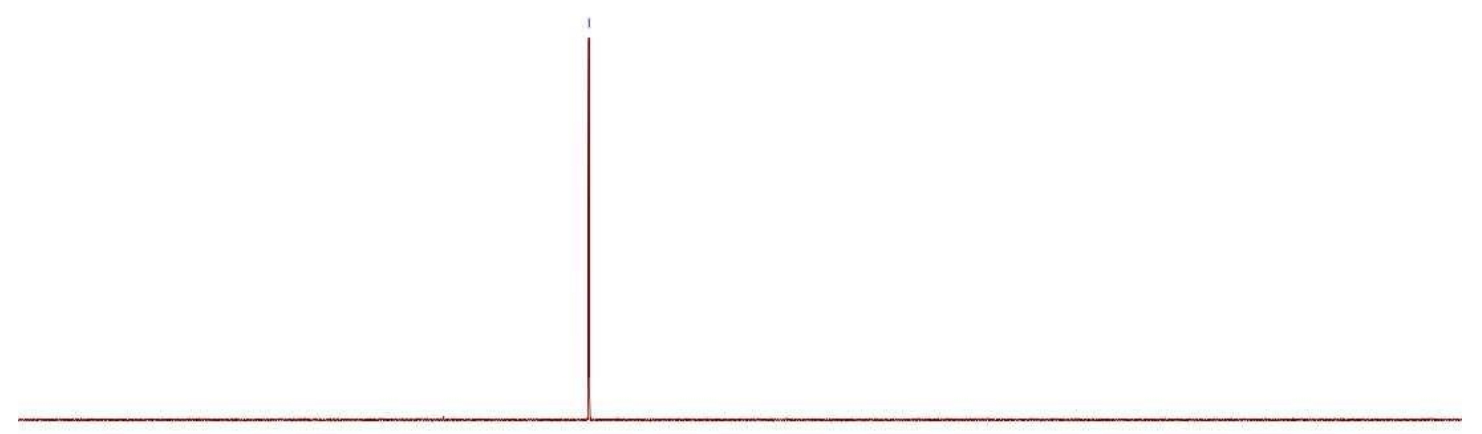
$\begin{array}{llllllllllllllllllllllllll}130 & 110 & 90 & 80 & 70 & 60 & 50 & 40 & 30 & 20 & 10 & 0 & -10 & -30 & -50 & -70 & -90 & -110 & -130 & -150 & -170 & -190 & -210 & -230 & 1 & -1\end{array}$

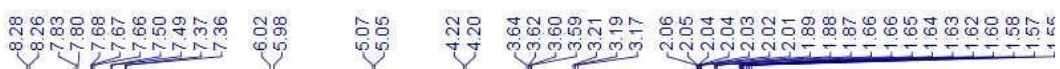
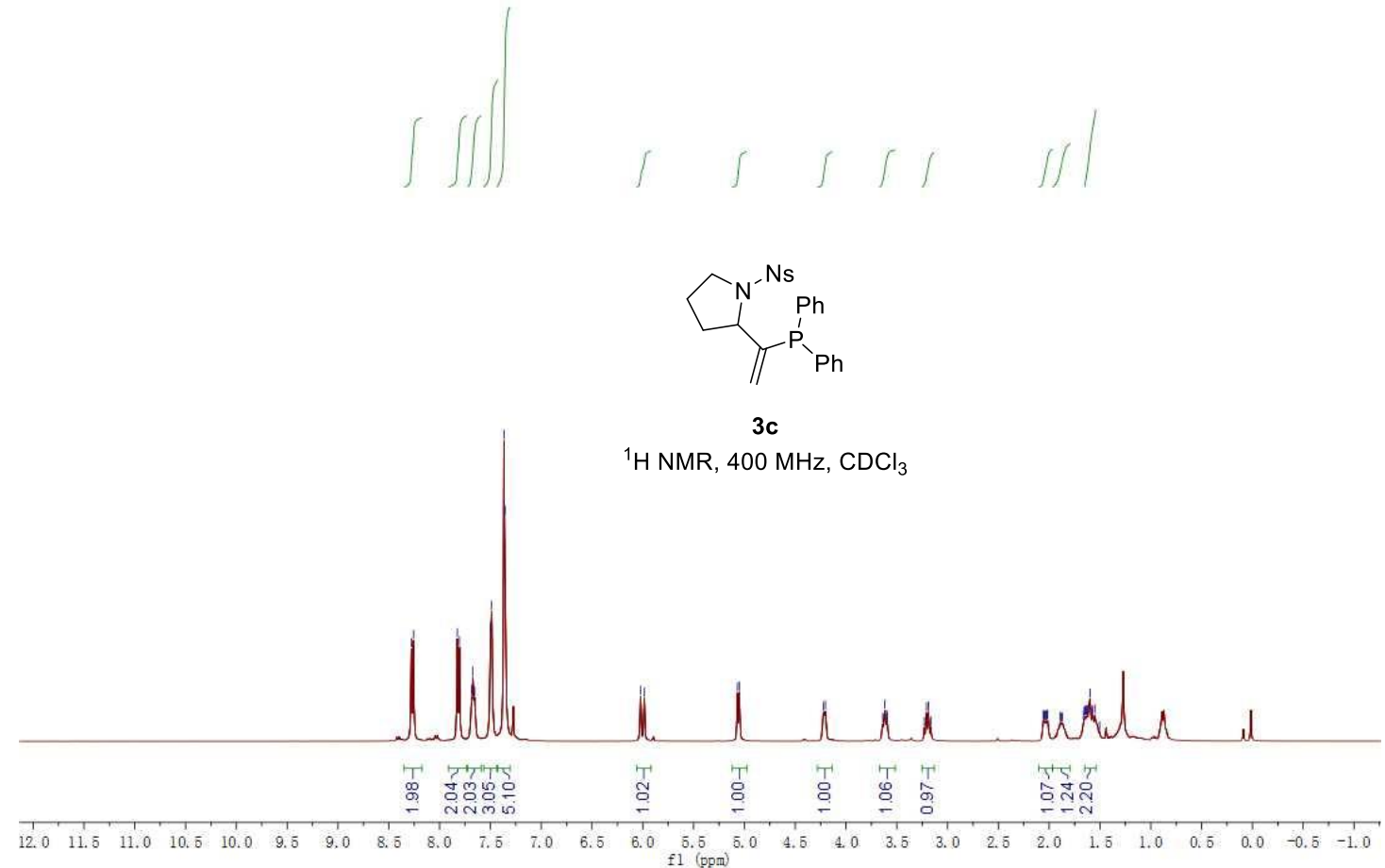


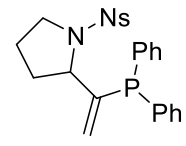

3c

${ }^{13} \mathrm{C}\left\{{ }^{1} \mathrm{H}\right\} \mathrm{NMR}, 100 \mathrm{MHz}, \mathrm{CDCl}_{3}$
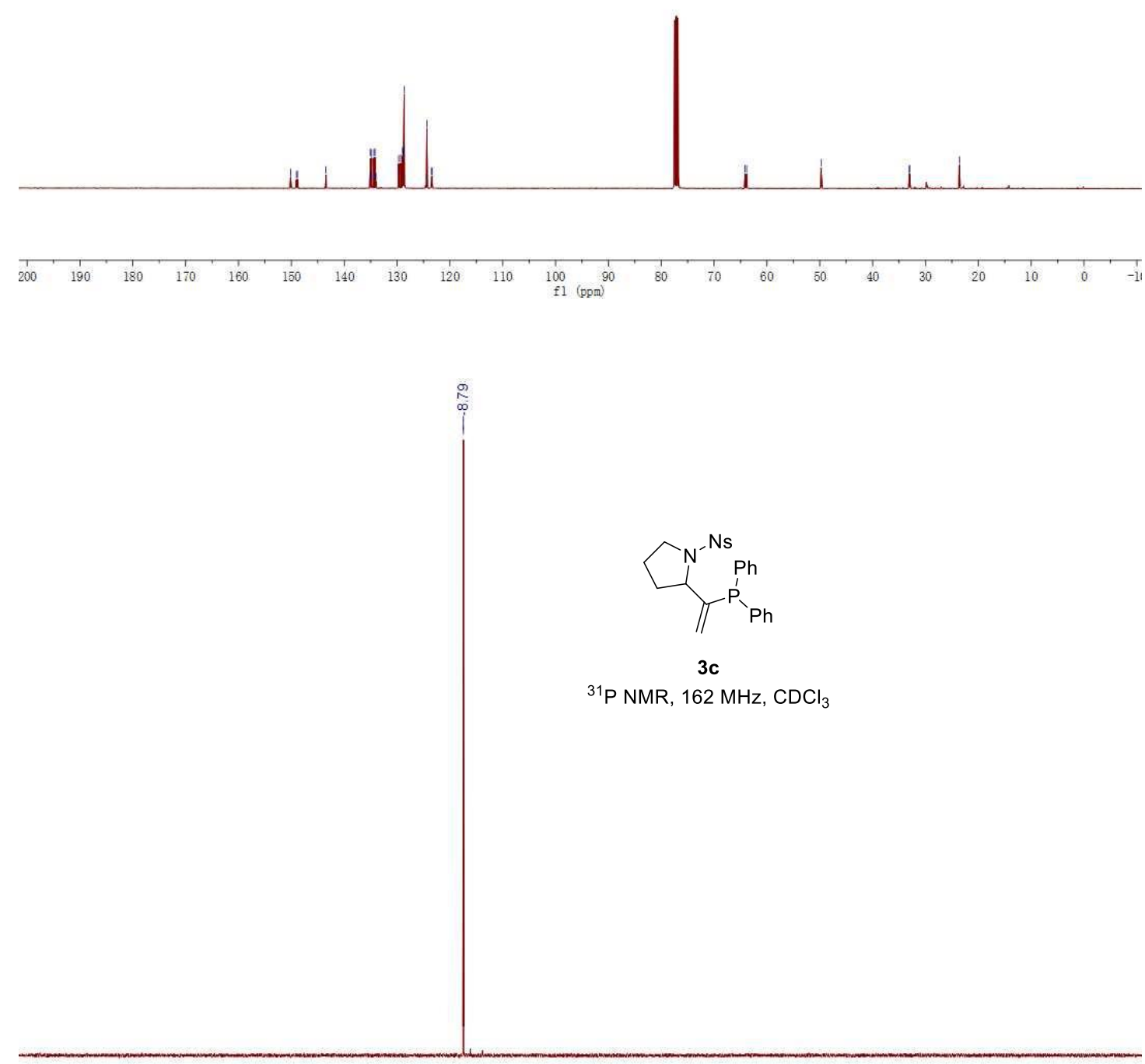

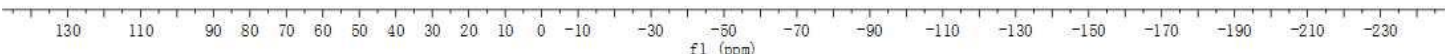




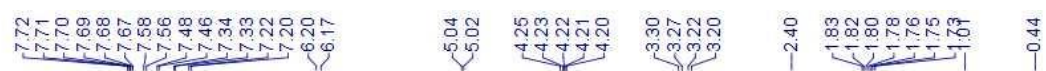
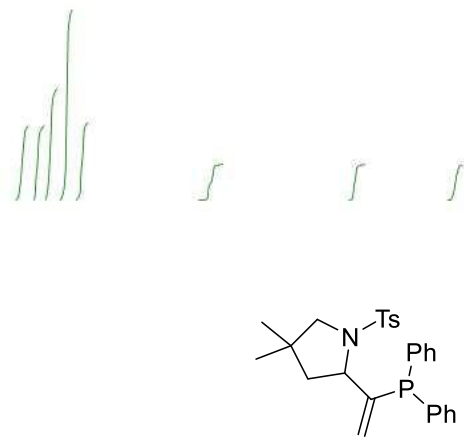

3d

${ }^{1} \mathrm{H}$ NMR, $400 \mathrm{MHz}, \mathrm{CDCl}_{3}$
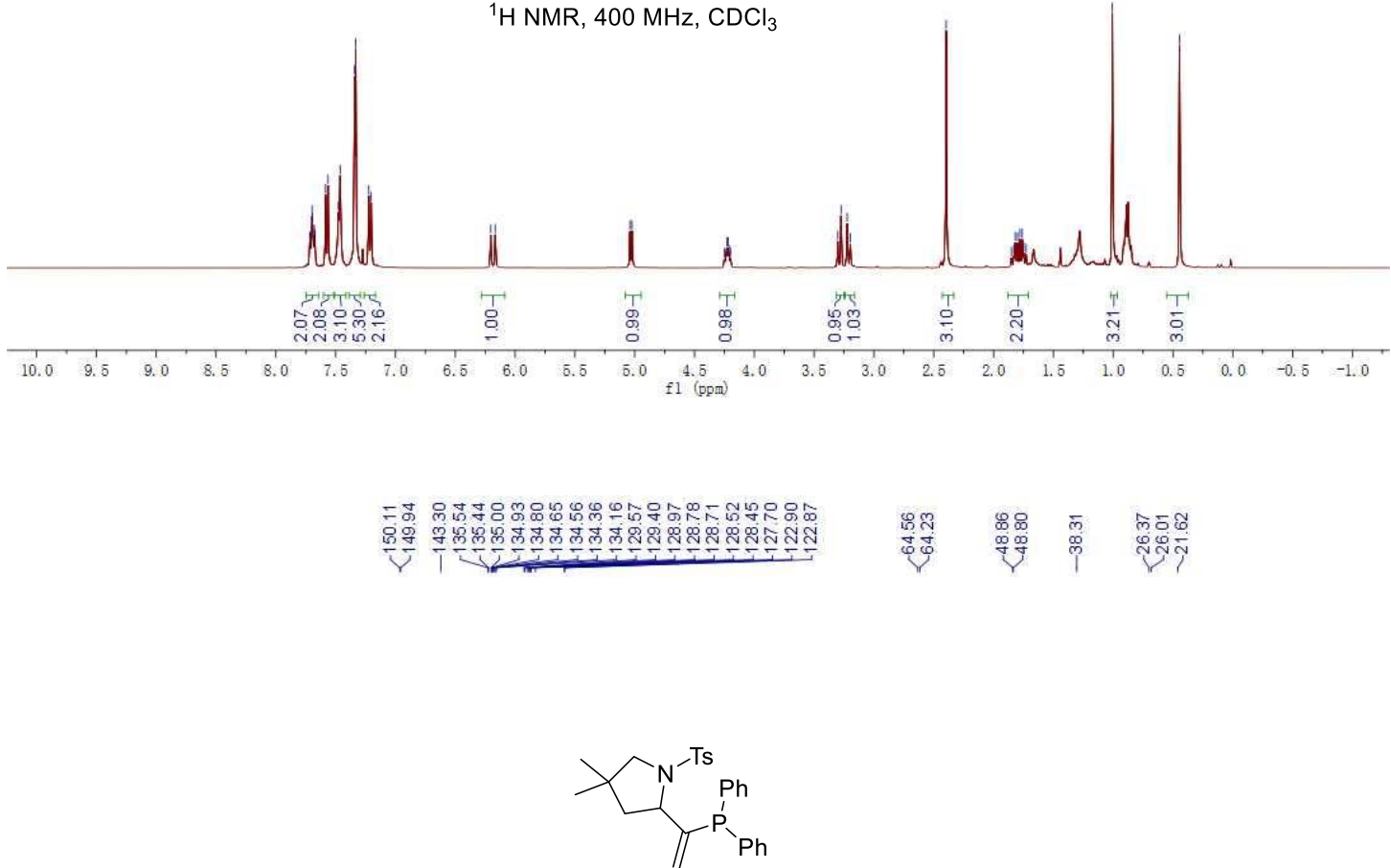

3d

${ }^{13} \mathrm{C}\left\{{ }^{1} \mathrm{H}\right\} \mathrm{NMR}, 100 \mathrm{MHz}, \mathrm{CDCl}_{3}$

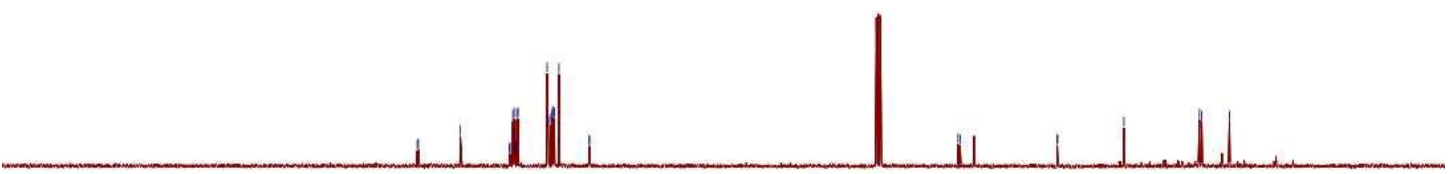

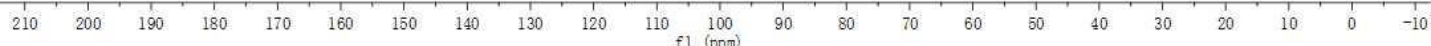




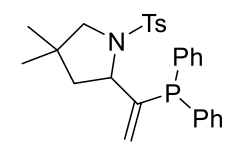

$3 \mathbf{d}$

${ }^{31} \mathrm{P} \mathrm{NMR}, 162 \mathrm{MHz}, \mathrm{CDCl}_{3}$

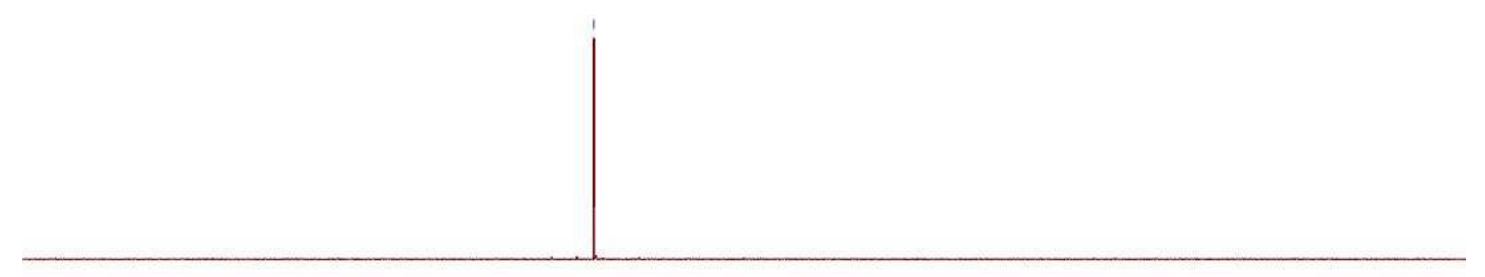

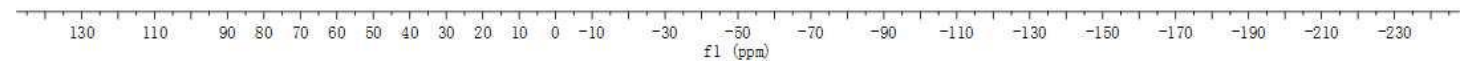

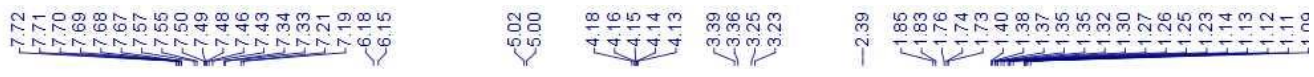
$\iiint l$

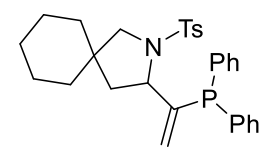

$3 e$

${ }^{1} \mathrm{H}$ NMR, $400 \mathrm{MHz}, \mathrm{CDCl}_{3}$

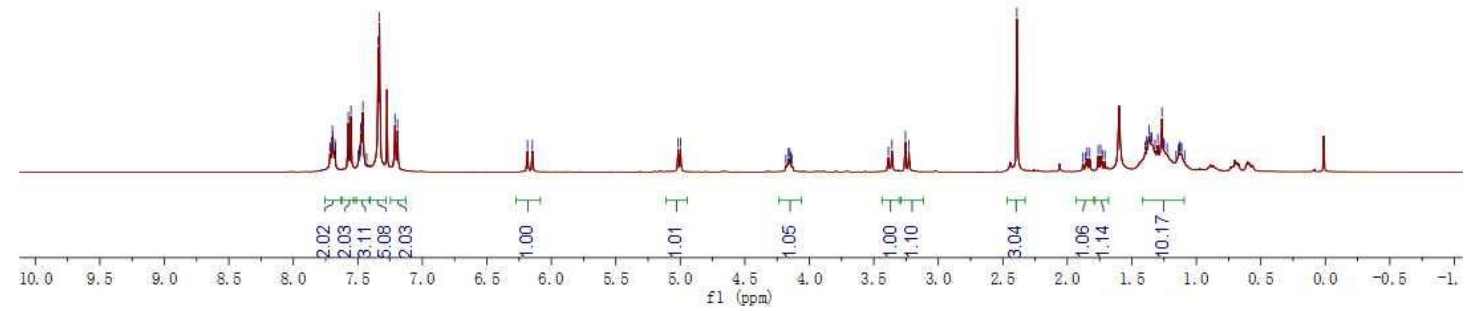



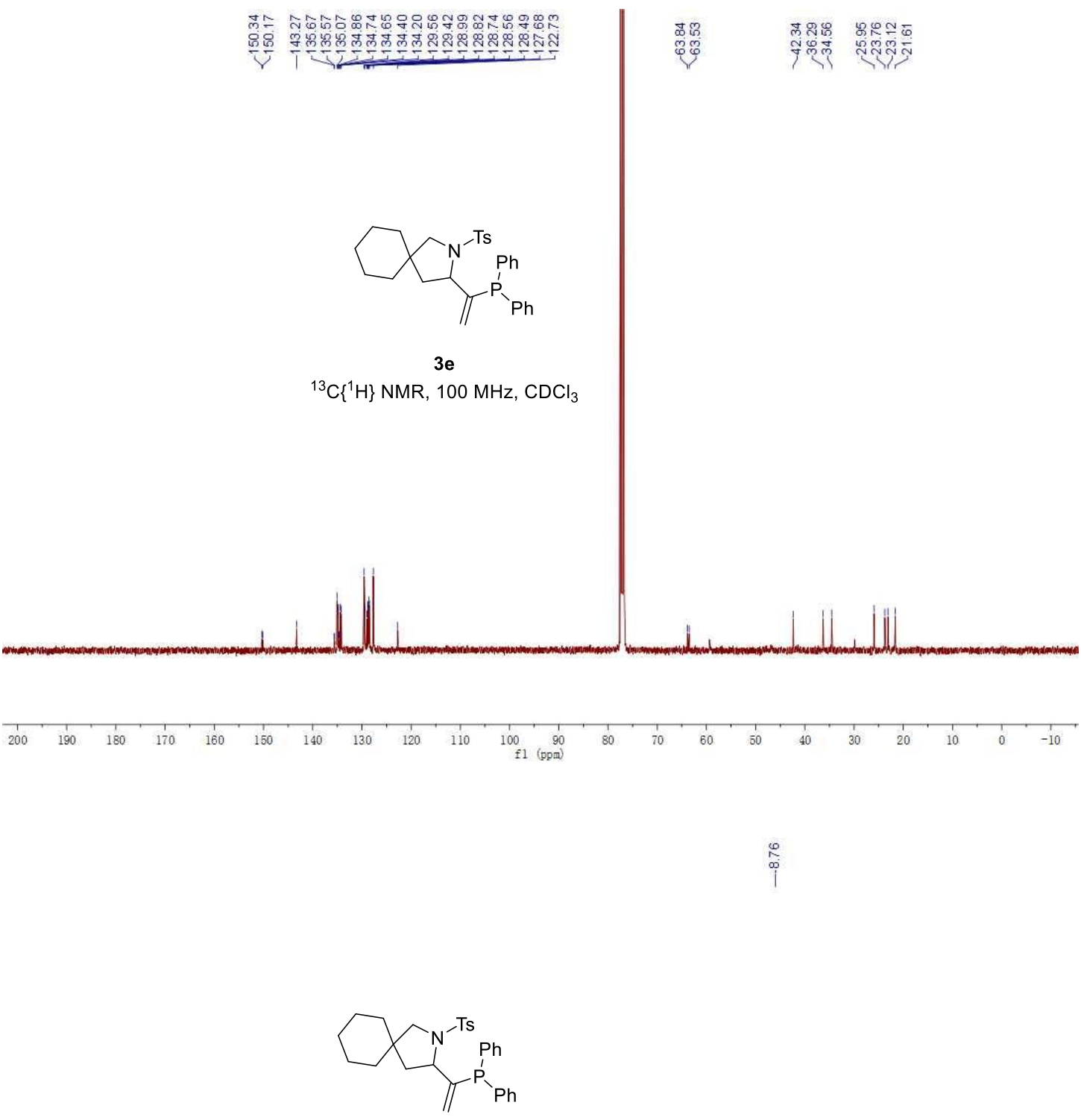

$3 e$

${ }^{31} \mathrm{P} \mathrm{NMR}, 162 \mathrm{MHz}, \mathrm{CDCl}_{3}$

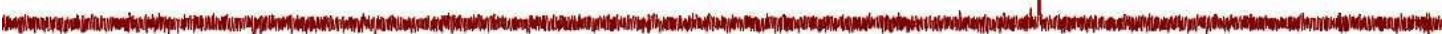

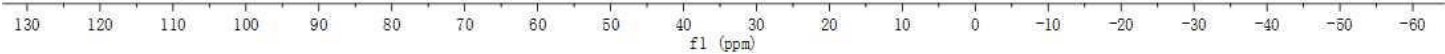


1

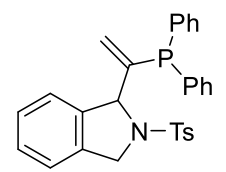

$5 a$

${ }^{1} \mathrm{H}$ NMR, $400 \mathrm{MHz}, \mathrm{CDCl}_{3}$

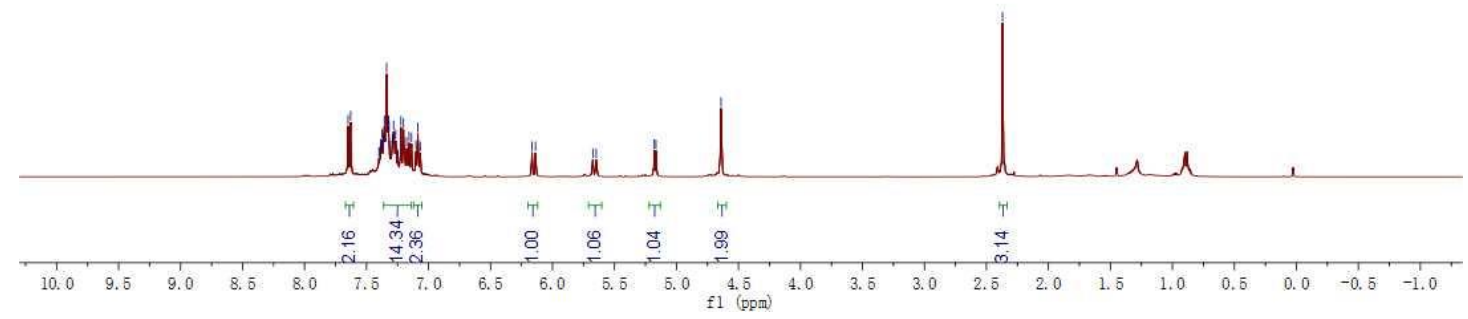

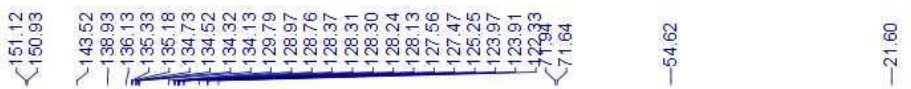

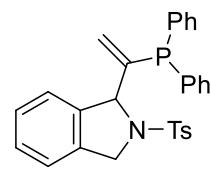

$5 a$

${ }^{13} \mathrm{C}\left\{{ }^{1} \mathrm{H}\right\} \mathrm{NMR}, 100 \mathrm{MHz}, \mathrm{CDCl}_{3}$

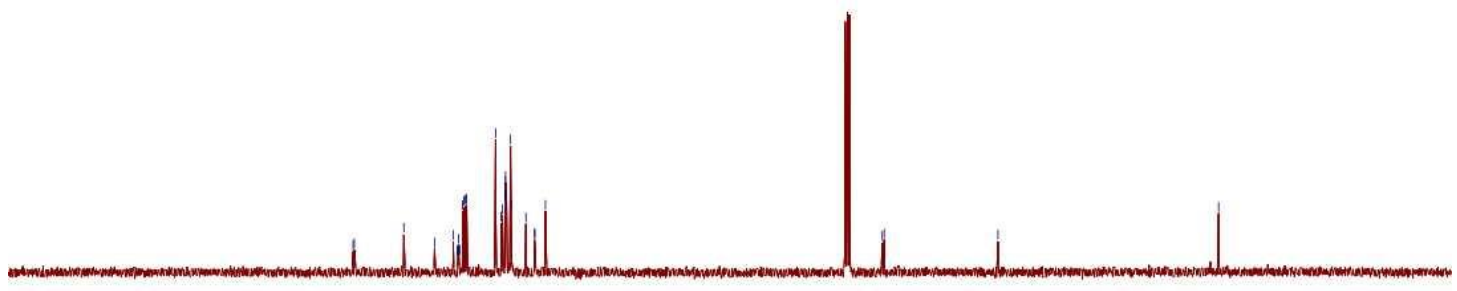

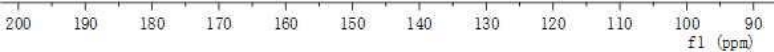




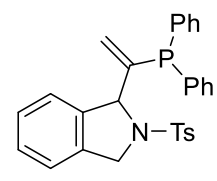

$5 a$

${ }^{31} \mathrm{P}$ NMR, $162 \mathrm{MHz}, \mathrm{CDCl}_{3}$

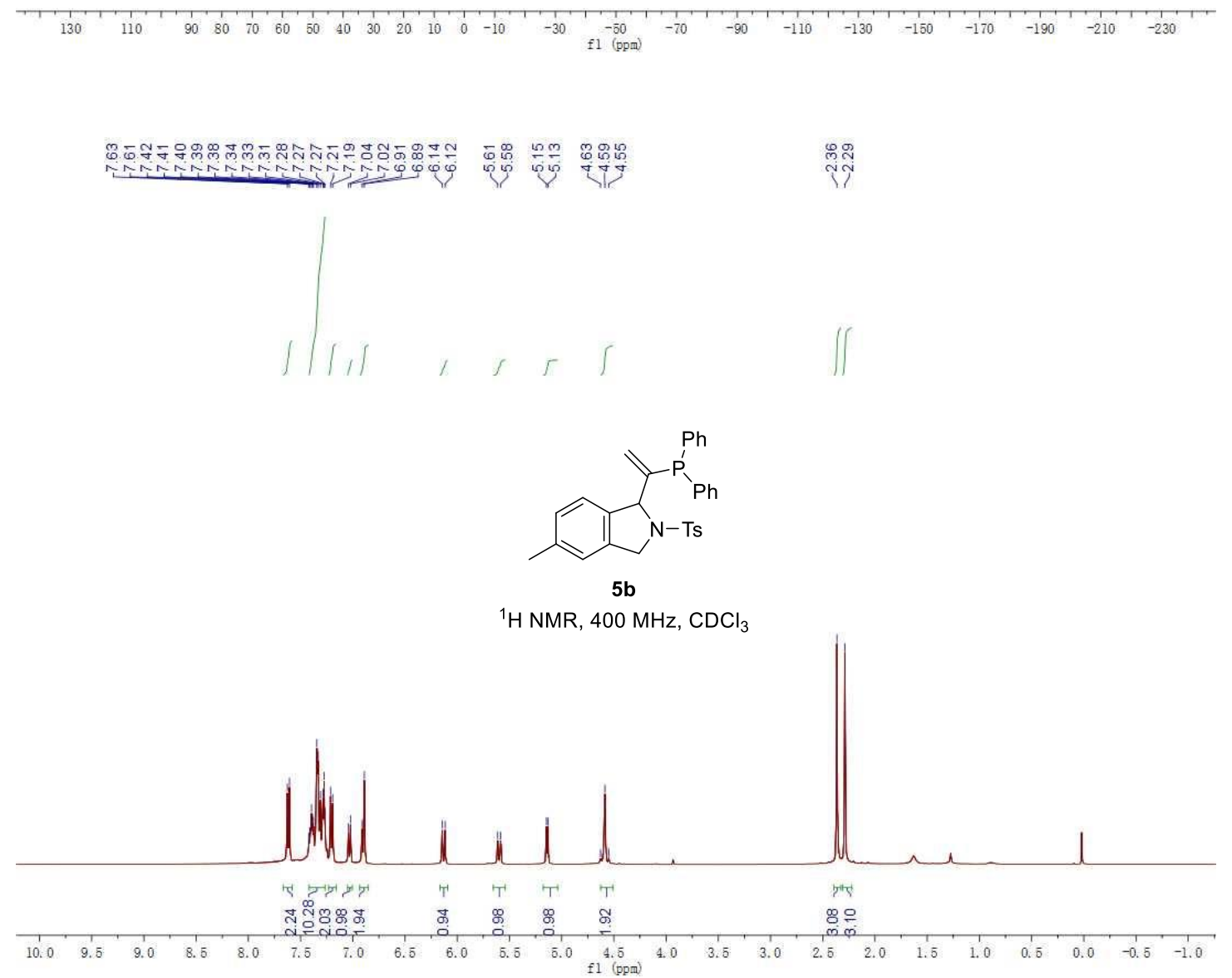




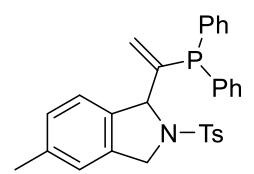

$5 \mathbf{b}$

$\left.{ }^{13} \mathrm{C}^{1}{ }^{1} \mathrm{H}\right\} \mathrm{NMR}, 100 \mathrm{MHz}, \mathrm{CDCl}_{3}$
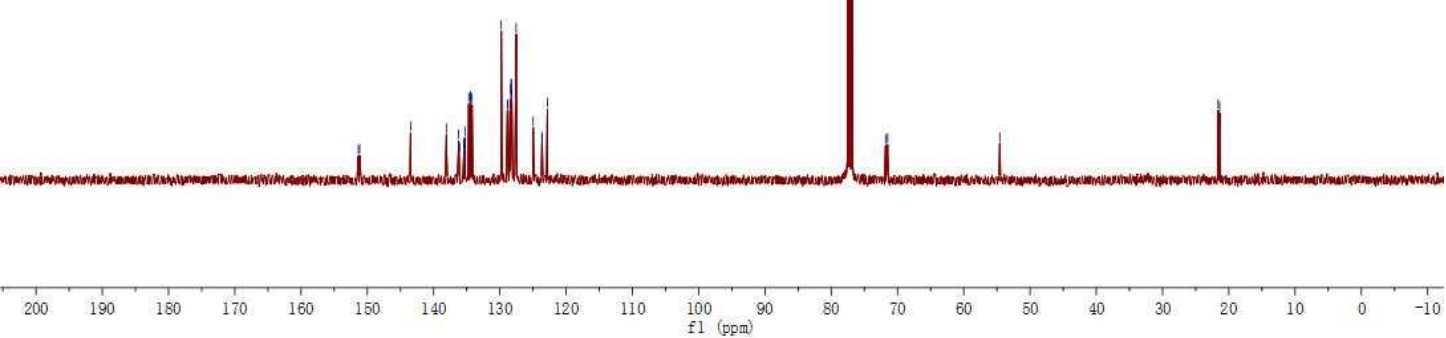

$\frac{\text { in }}{i}$

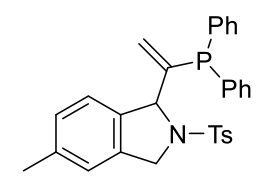

$5 b$

${ }^{31} \mathrm{P} \mathrm{NMR}, 162 \mathrm{MHz}, \mathrm{CDCl}_{3}$

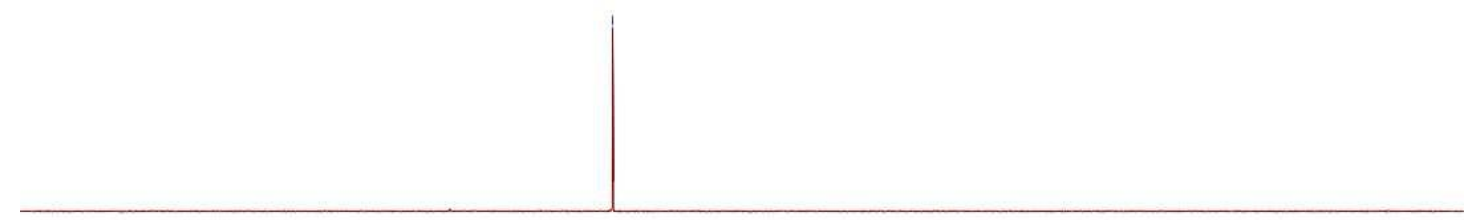

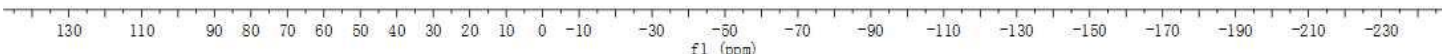




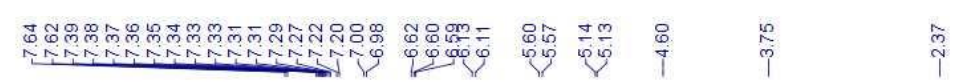
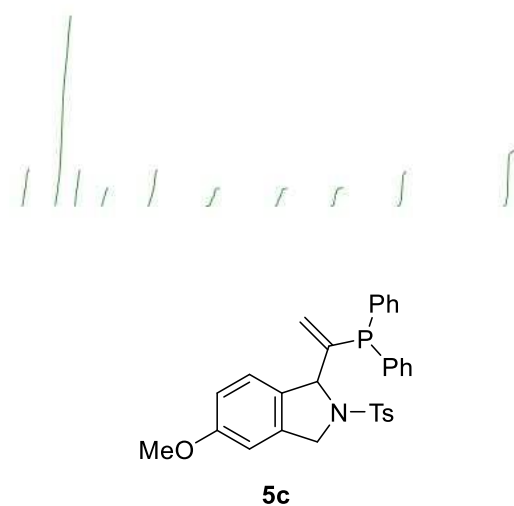

${ }^{1} \mathrm{H}$ NMR, $400 \mathrm{MHz}, \mathrm{CDCl}_{3}$

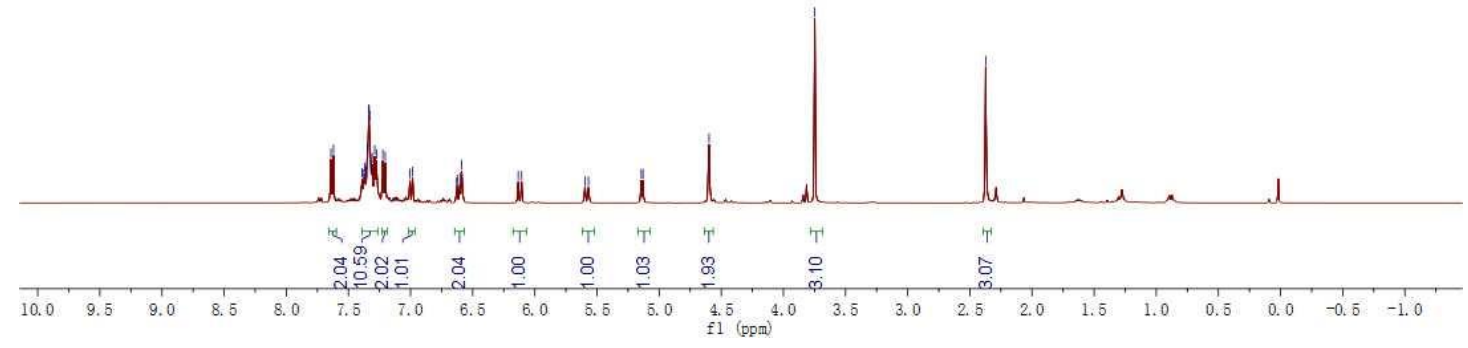

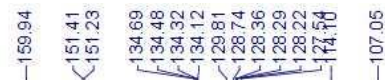

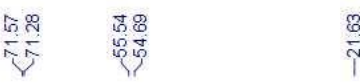

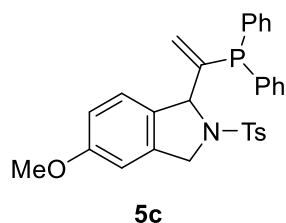

${ }^{13} \mathrm{C}\left\{{ }^{1} \mathrm{H}\right\} \mathrm{NMR}, 100 \mathrm{MHz}, \mathrm{CDCl}_{3}$

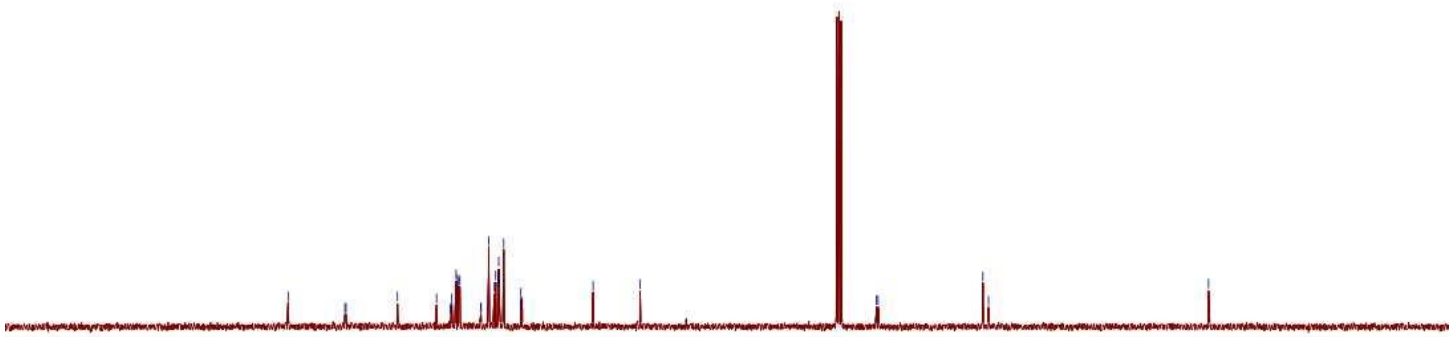

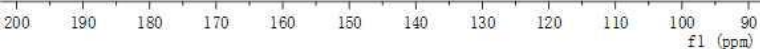




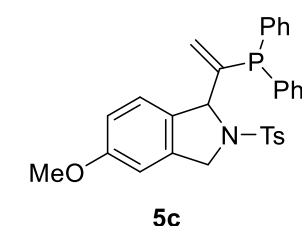

${ }^{31} \mathrm{P} \mathrm{NMR}, 162 \mathrm{MHz}, \mathrm{CDCl}_{3}$
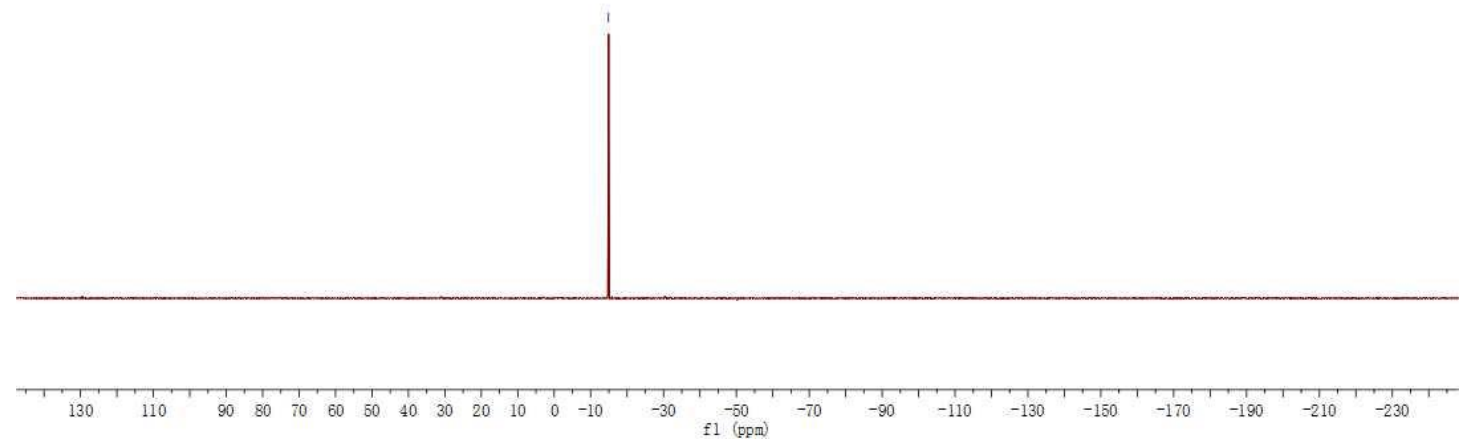

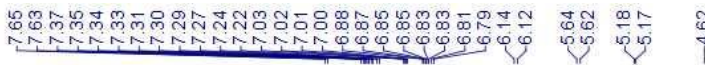
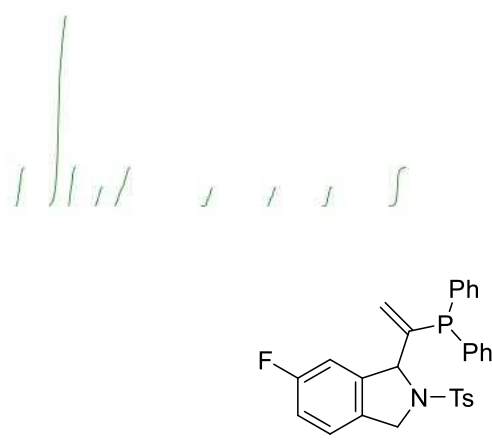

5d

${ }^{1} \mathrm{H} \mathrm{NMR}, 400 \mathrm{MHz}, \mathrm{CDCl}_{3}$

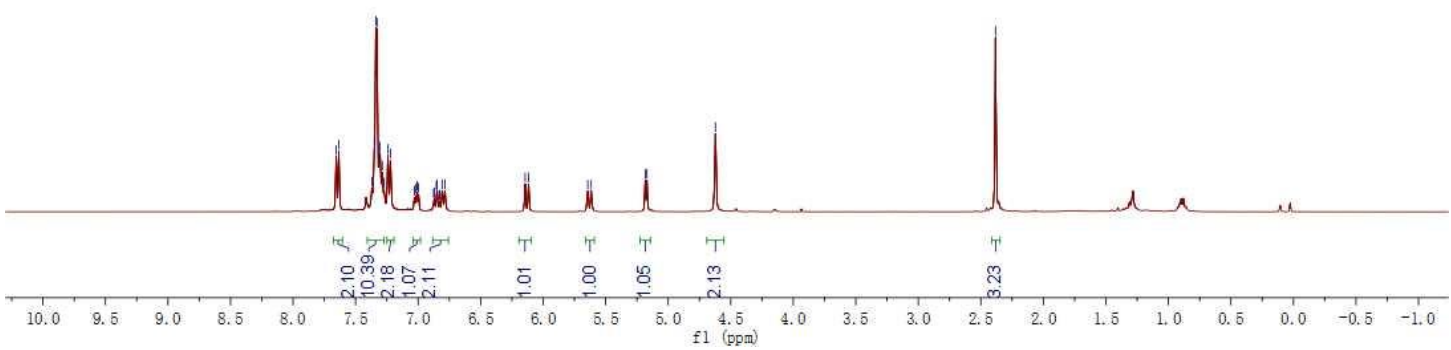




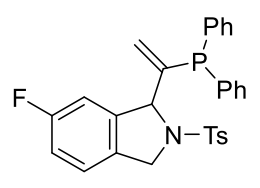

5d

${ }^{13} \mathrm{C}\left\{{ }^{1} \mathrm{H}\right\} \mathrm{NMR}, 100 \mathrm{MHz}, \mathrm{CDCl}_{3}$

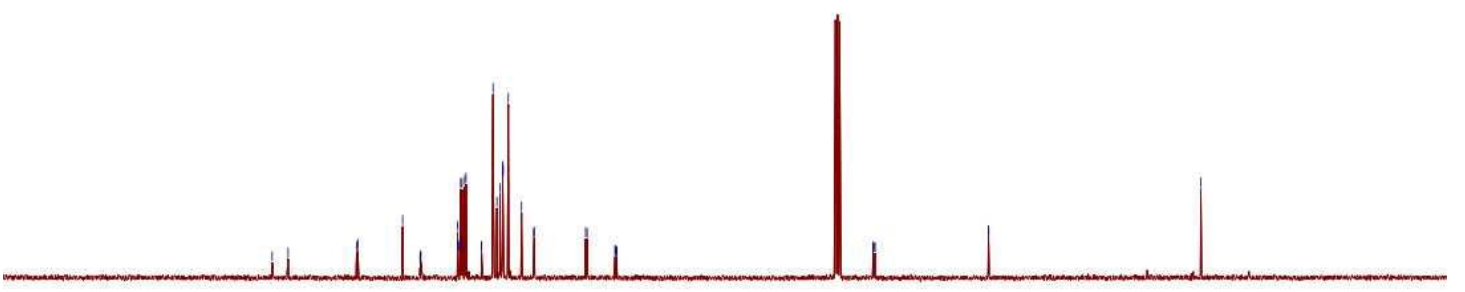

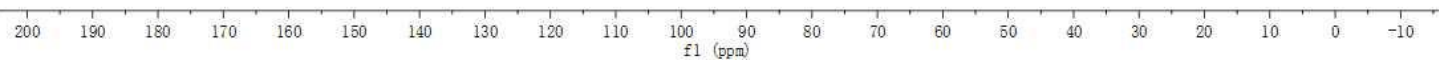

$\frac{i}{\frac{1}{4}}$

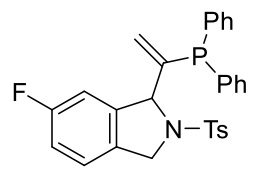

$5 d$

${ }^{31} \mathrm{P}$ NMR, $162 \mathrm{MHz}, \mathrm{CDCl}_{3}$

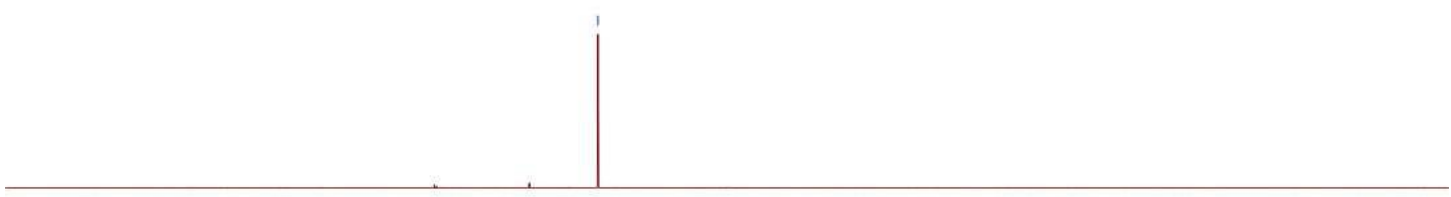

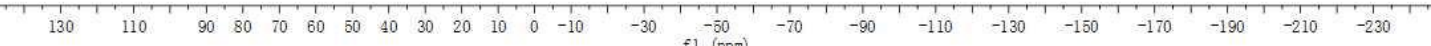




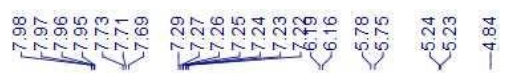
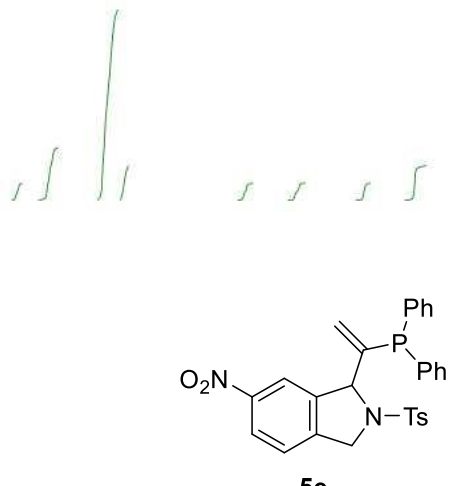

${ }^{1} \mathrm{H}$ NMR, $400 \mathrm{MHz}, \mathrm{CDCl}_{3}$

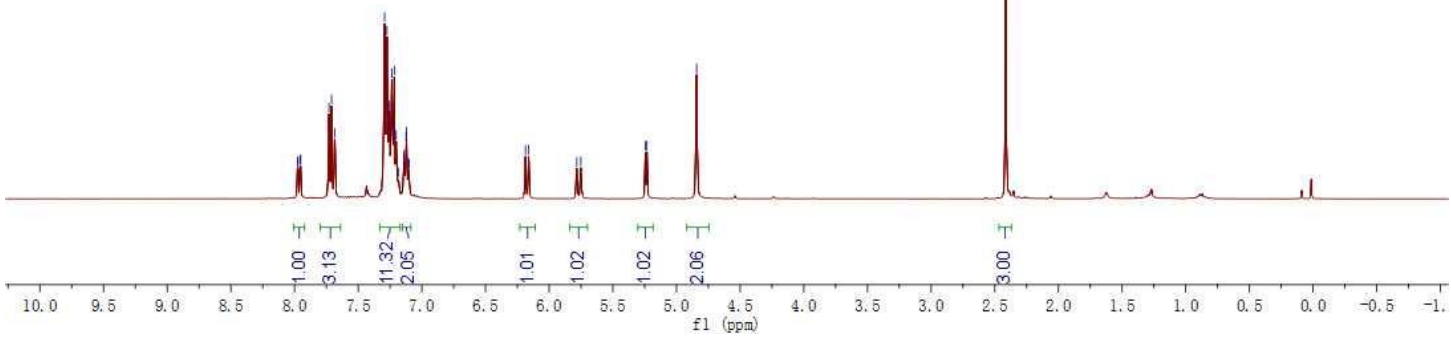

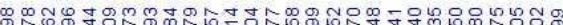

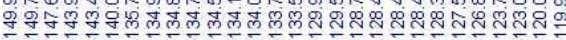

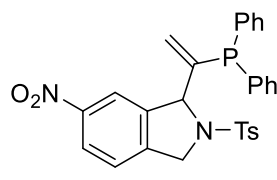

5 e

${ }^{13} \mathrm{C}\left\{{ }^{1} \mathrm{H}\right\} \mathrm{NMR}, 100 \mathrm{MHz}, \mathrm{CDCl}_{3}$

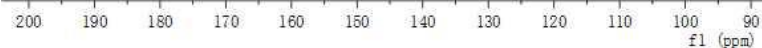




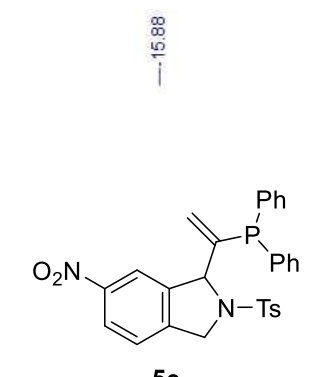

${ }^{31} \mathrm{P}$ NMR, $162 \mathrm{MHz}, \mathrm{CDCl}_{3}$

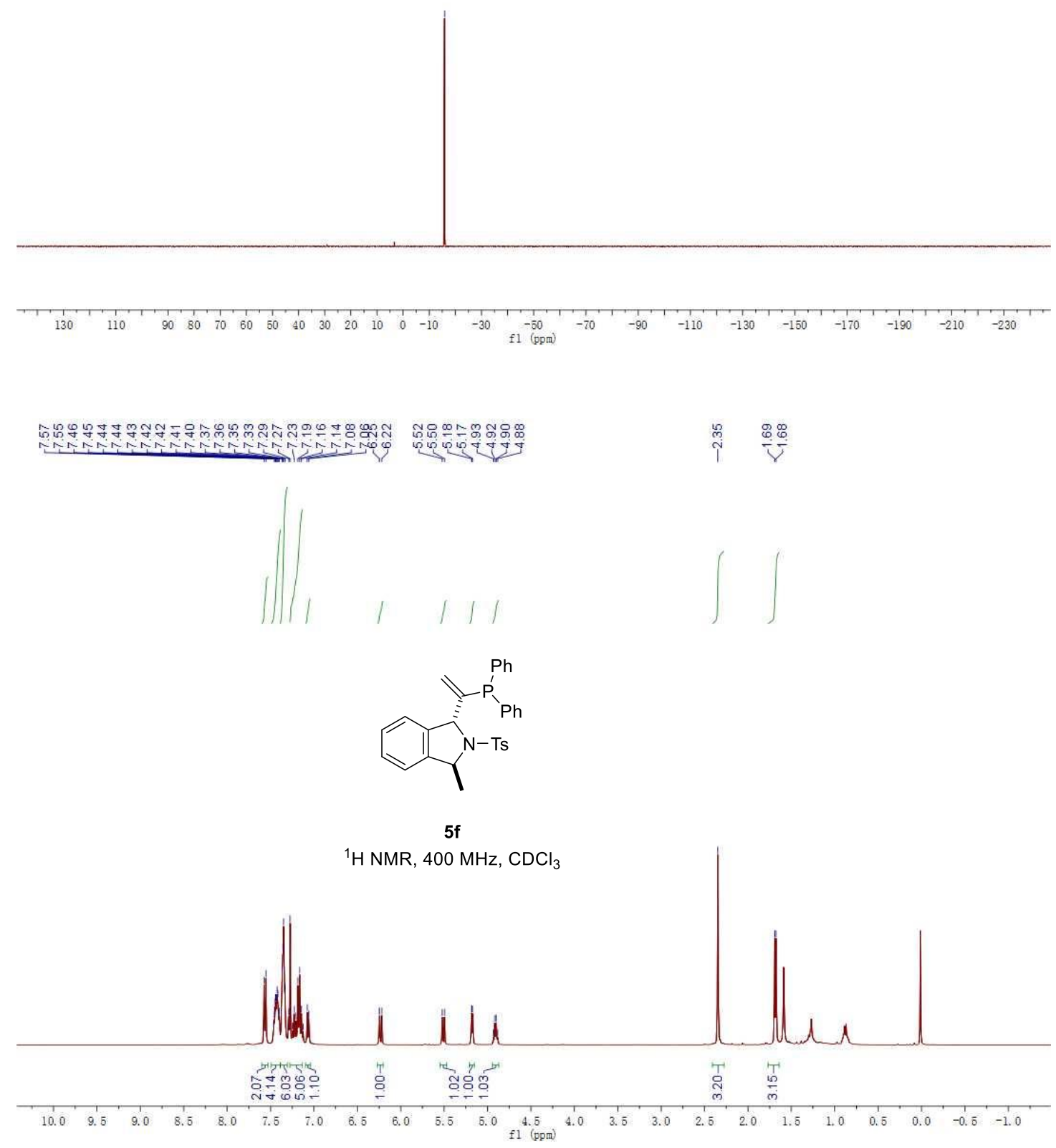




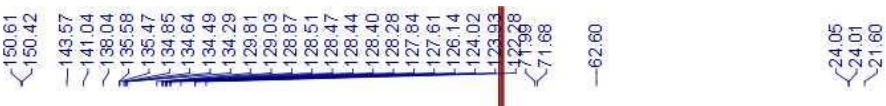

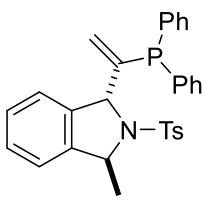

$5 f$

${ }^{13} \mathrm{C}\left\{{ }^{1} \mathrm{H}\right\} \mathrm{NMR}, 100 \mathrm{MHz}, \mathrm{CDCl}_{3}$
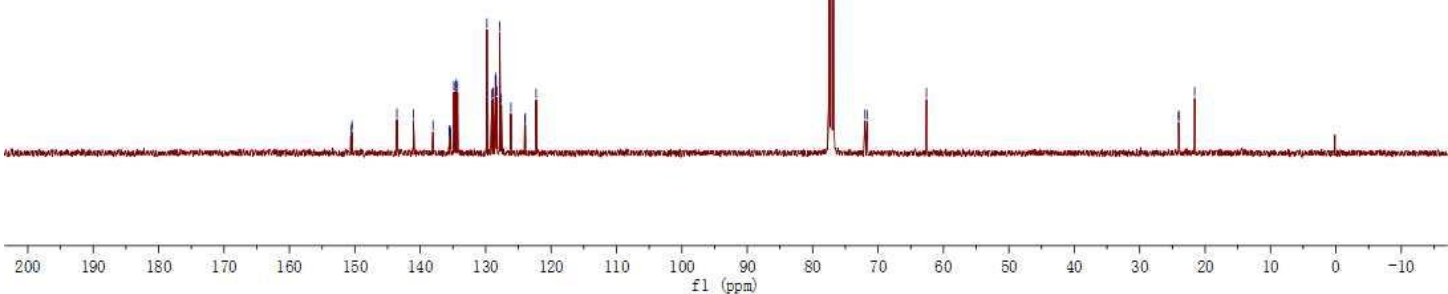

$\frac{8}{i}$

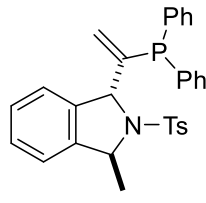

$5 f$

${ }^{31} \mathrm{P} \mathrm{NMR}, 162 \mathrm{MHz}, \mathrm{CDCl}_{3}$

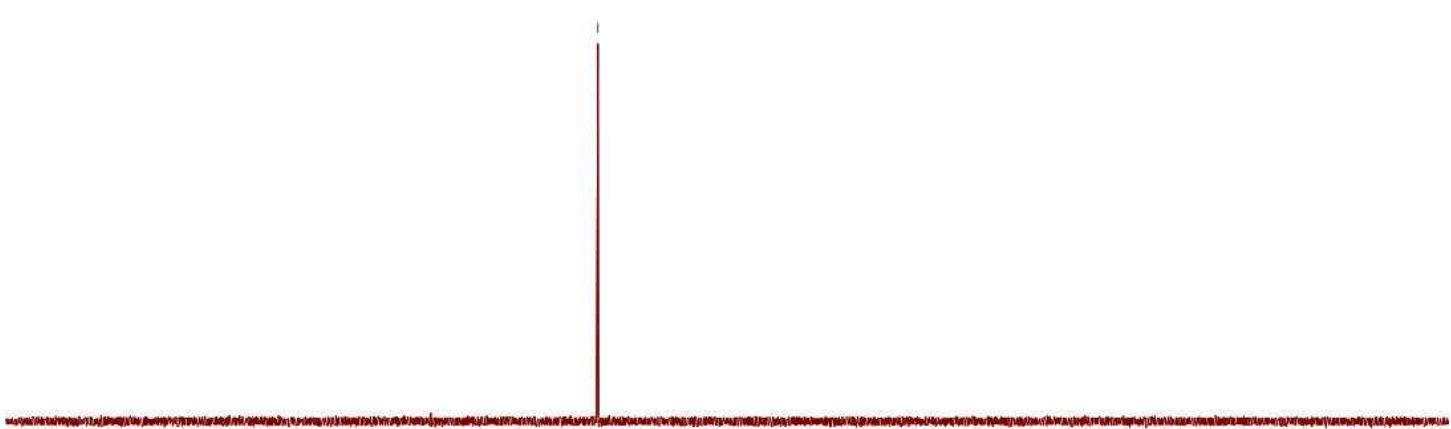

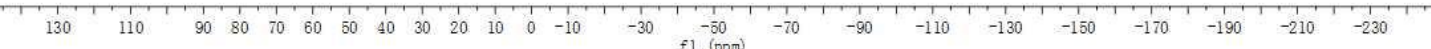




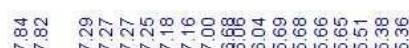

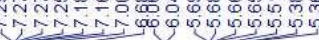

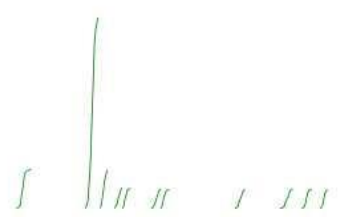

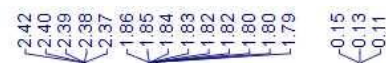

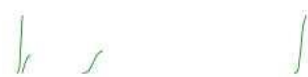<smiles>C=C(C1c2ccccc2C(CC)N1C)P(c1ccccc1)c1ccccc1</smiles>

$5 \mathrm{~g}$

${ }^{1} \mathrm{H}$ NMR, $400 \mathrm{MHz}, \mathrm{CDCl}_{3}$

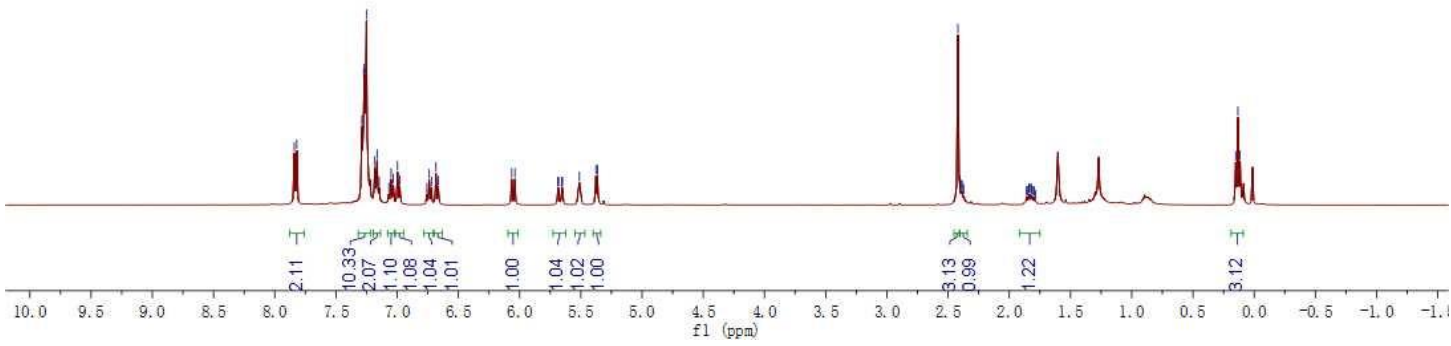

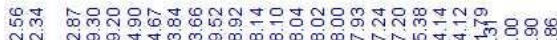

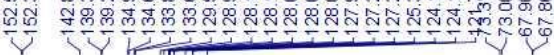

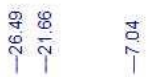

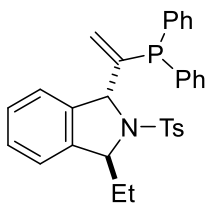

$5 \mathrm{~g}$

${ }^{13} \mathrm{C}\left\{{ }^{1} \mathrm{H}\right\}$ NMR, $100 \mathrm{MHz}, \mathrm{CDCl}_{3}$

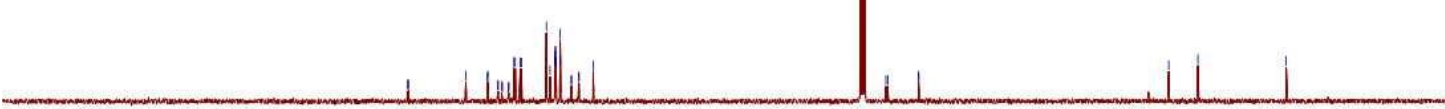

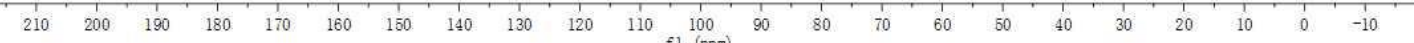




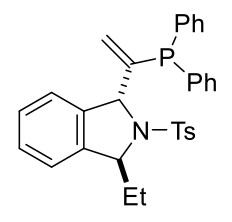

$5 \mathrm{~g}$

${ }^{31} \mathrm{P} \mathrm{NMR}, 162 \mathrm{MHz}, \mathrm{CDCl}_{3}$

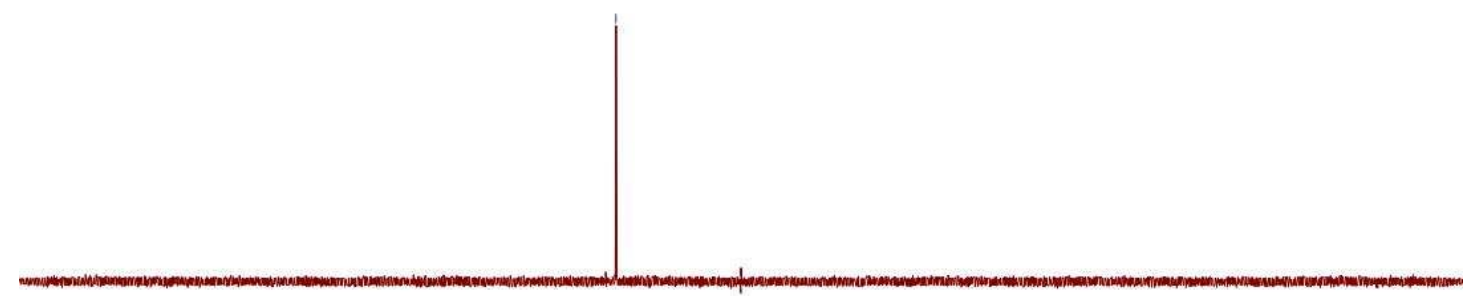

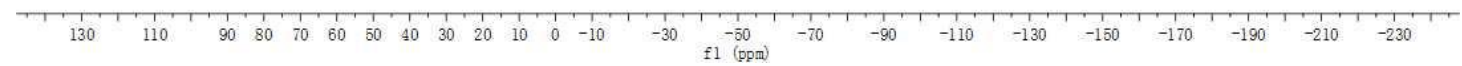
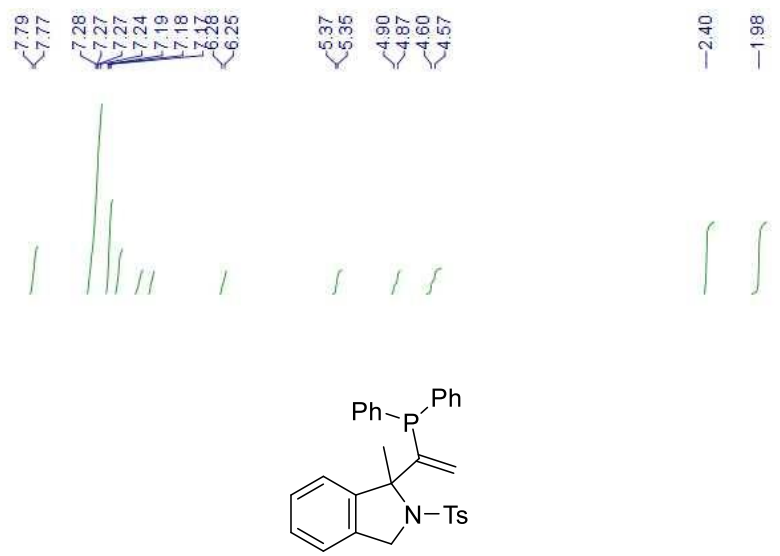

5 h

${ }^{1} \mathrm{H}$ NMR, $400 \mathrm{MHz}, \mathrm{CDCl}_{3}$

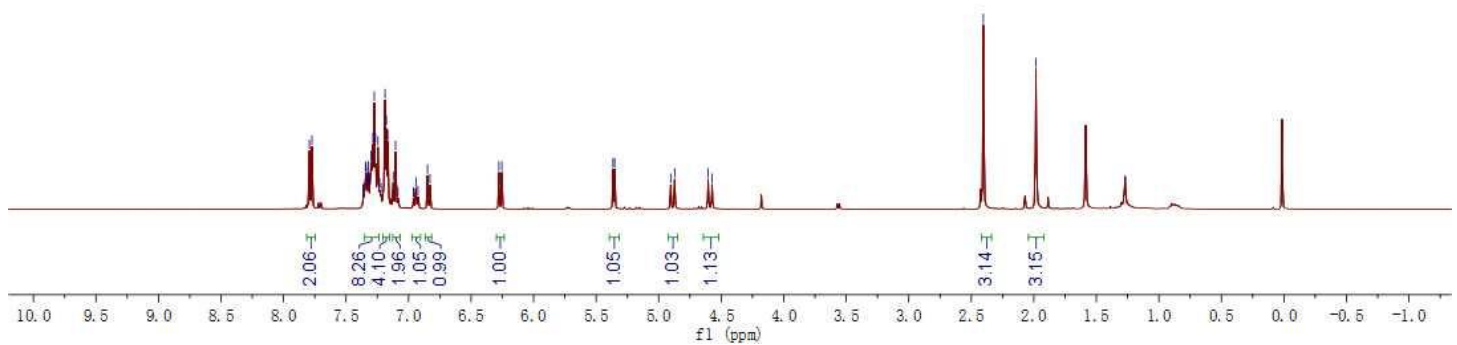



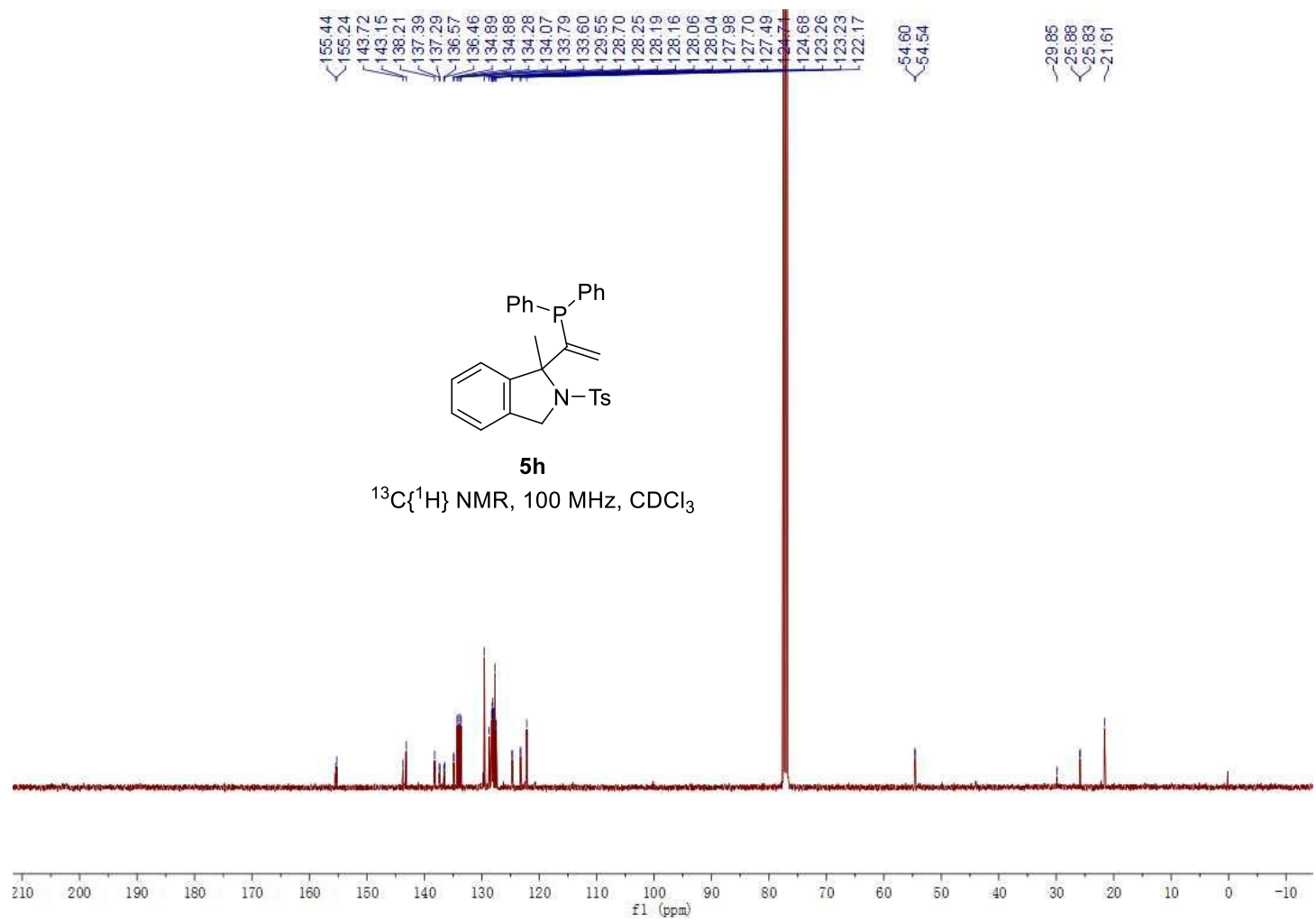

in

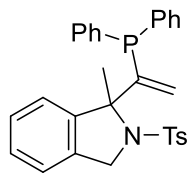

$5 \mathrm{~h}$

${ }^{31} \mathrm{P}$ NMR, $162 \mathrm{MHz}, \mathrm{CDCl}_{3}$

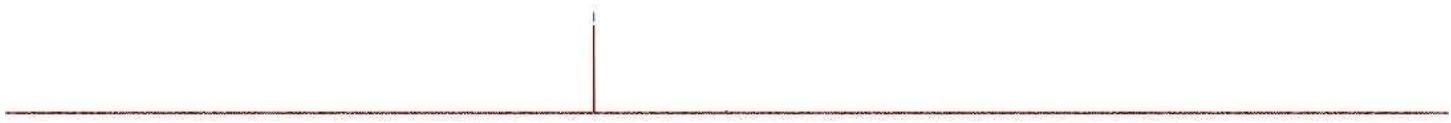

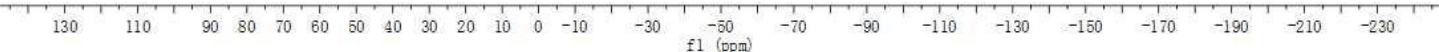




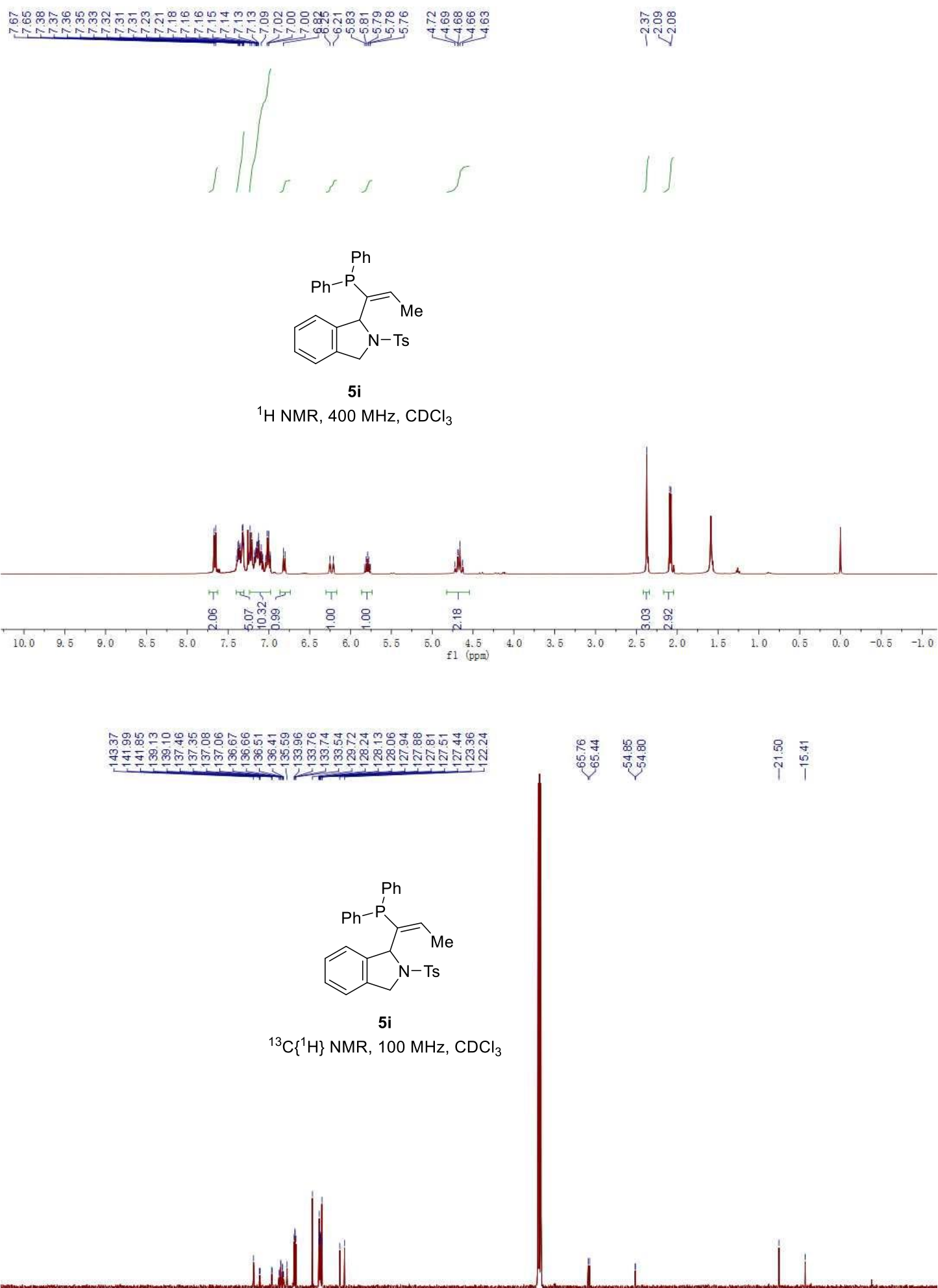

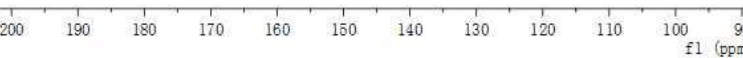




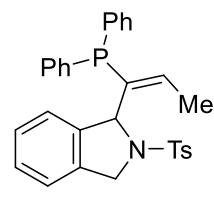

$5 i$

${ }^{31} \mathrm{P}$ NMR, $162 \mathrm{MHz}, \mathrm{CDCl}_{3}$
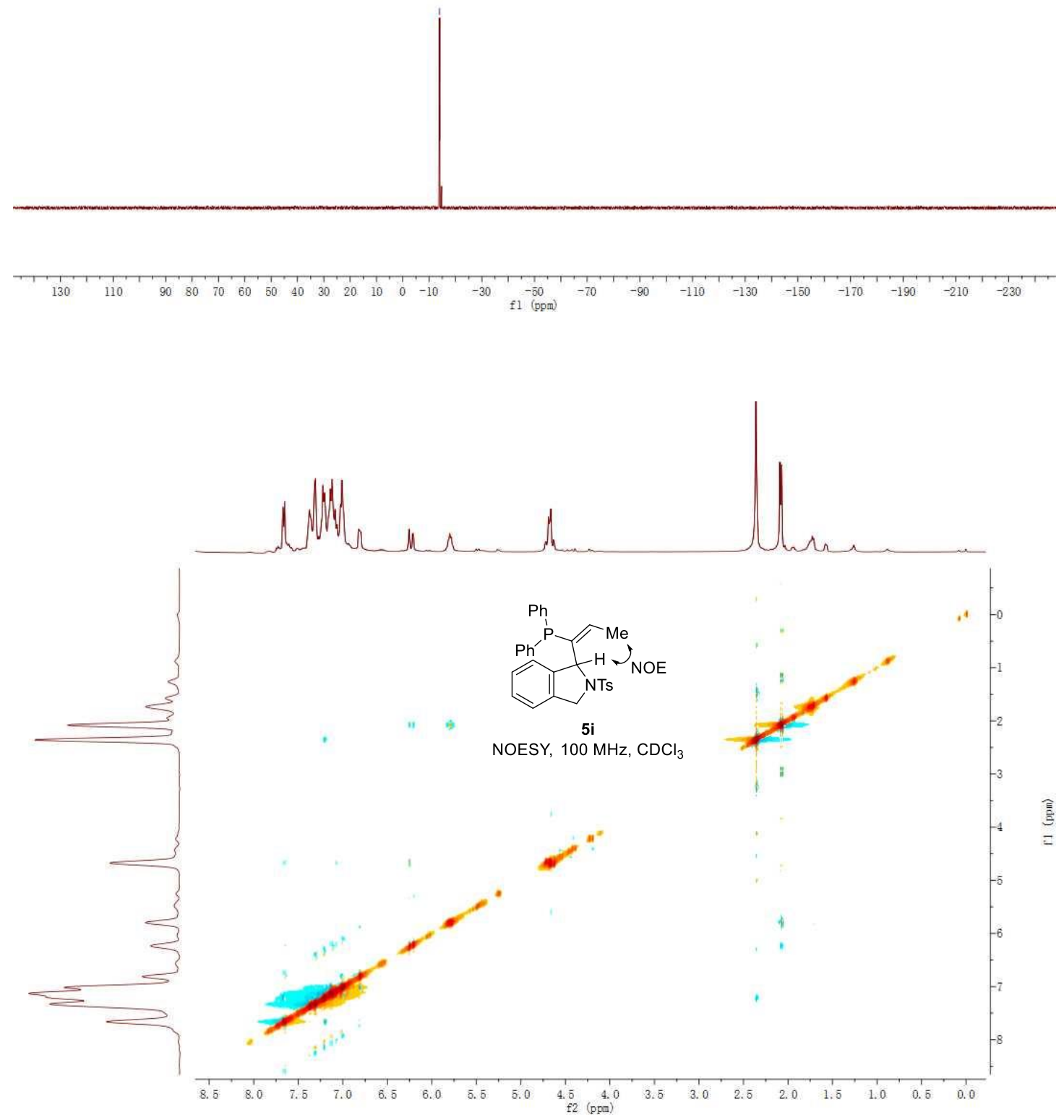


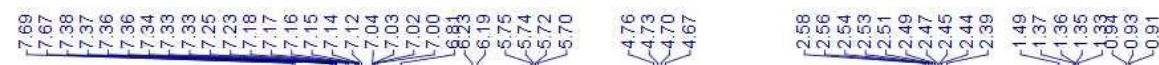

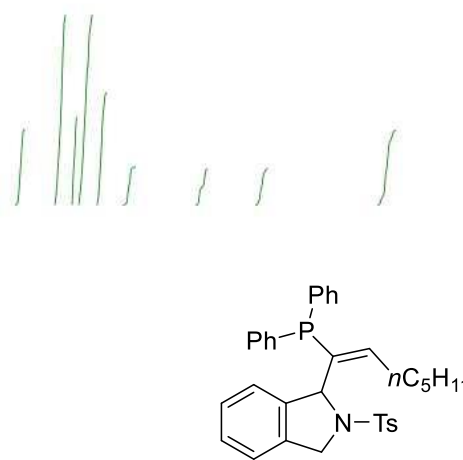

$5 \mathbf{j}$

${ }^{1} \mathrm{H}$ NMR, $400 \mathrm{MHz}, \mathrm{CDCl}_{3}$

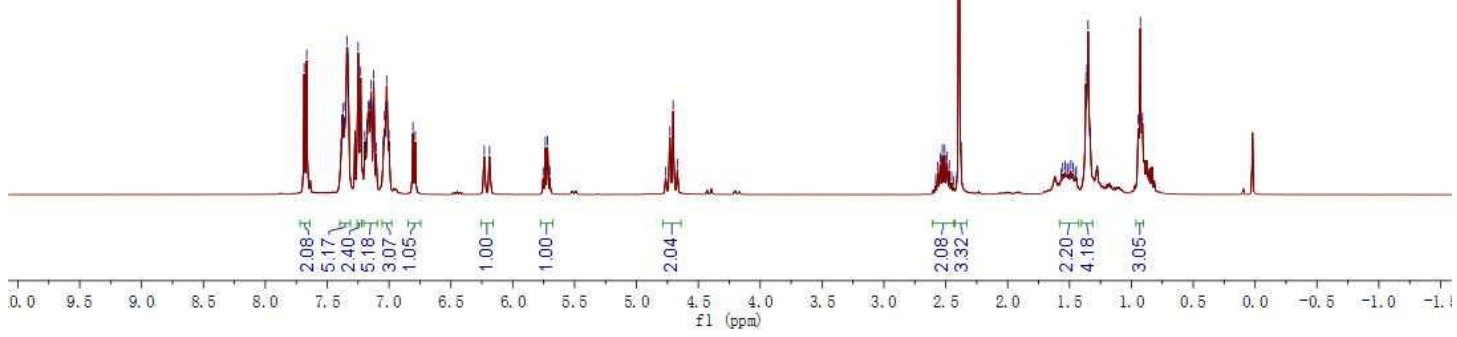

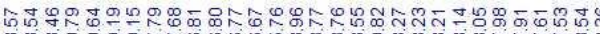

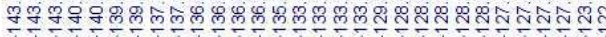

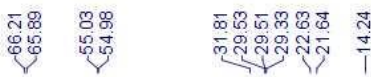

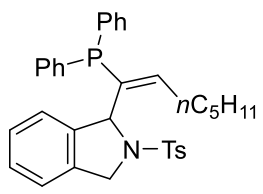

$5 \mathbf{j}$

${ }^{13} \mathrm{C}\left\{{ }^{1} \mathrm{H}\right\} \mathrm{NMR}, 100 \mathrm{MHz}, \mathrm{CDCl}_{3}$

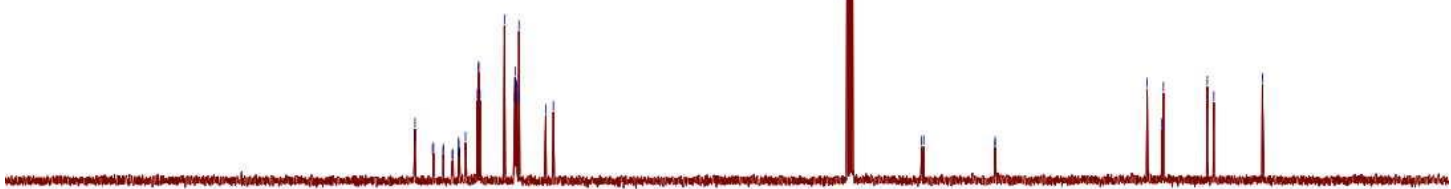

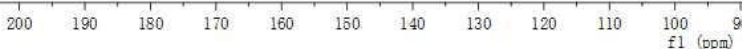




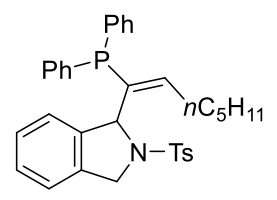

5j

${ }^{31} \mathrm{P} \mathrm{NMR}, 162 \mathrm{MHz}, \mathrm{CDCl}_{3}$

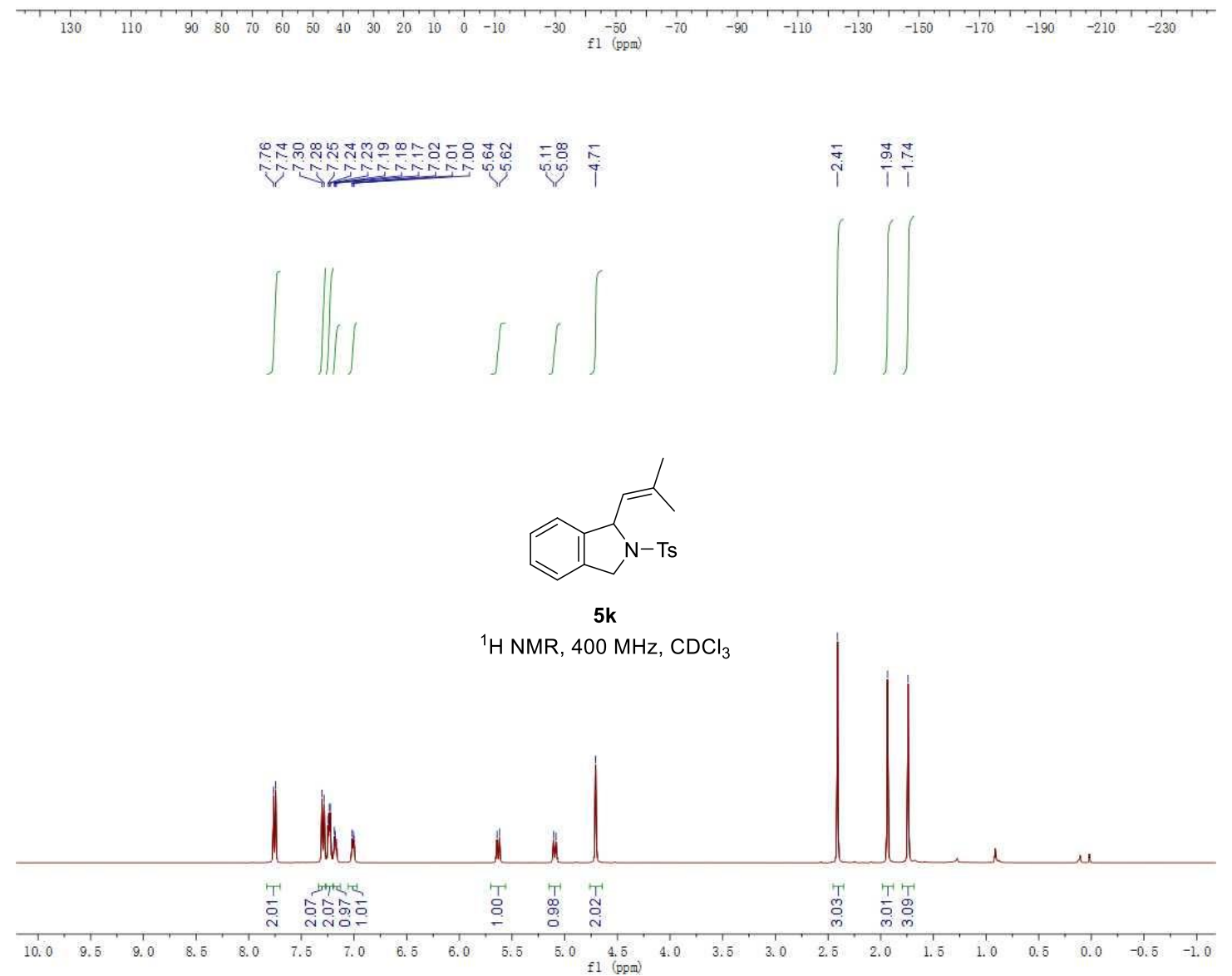




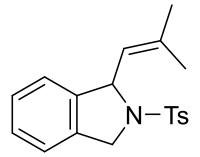

$5 k$

${ }^{13} \mathrm{C}\left\{{ }^{1} \mathrm{H}\right\} \mathrm{NMR}, 100 \mathrm{MHz}, \mathrm{CDCl}_{3}$

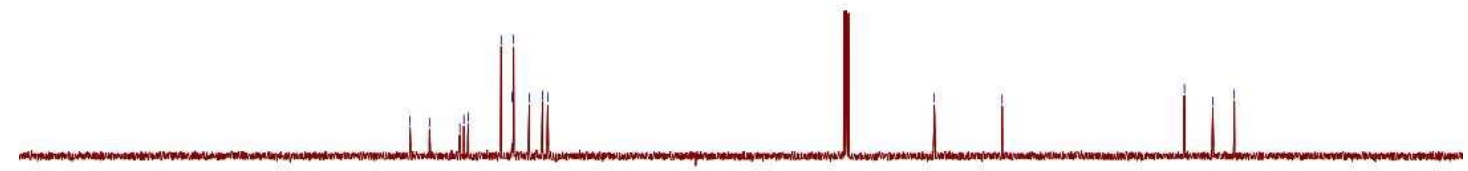

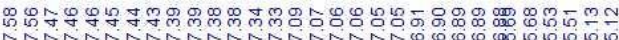

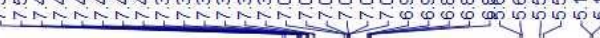
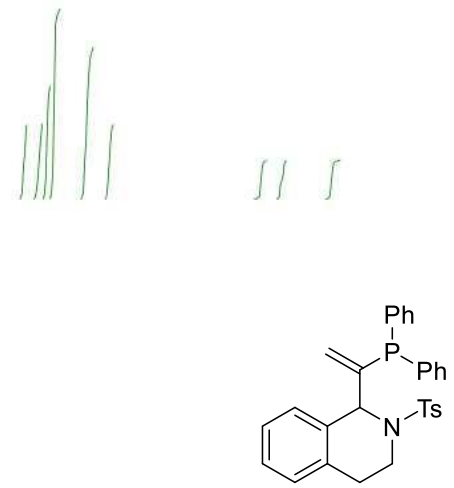

$5 I$

${ }^{1} \mathrm{H} \mathrm{NMR}, 400 \mathrm{MHz}, \mathrm{CDCl}_{3}$

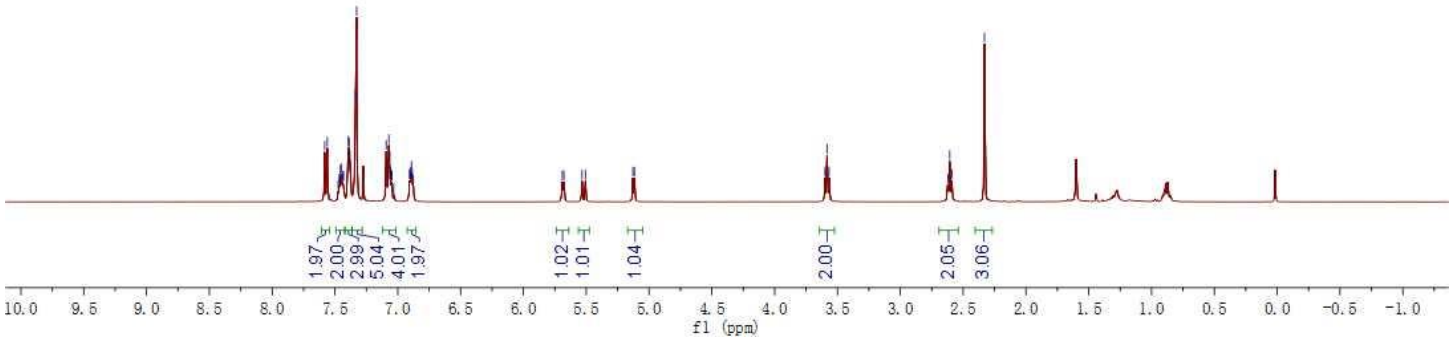




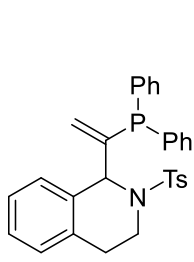

51

${ }^{13} \mathrm{C}\left\{{ }^{1} \mathrm{H}\right\} \mathrm{NMR}, 100 \mathrm{MHz}, \mathrm{CDCl}_{3}$
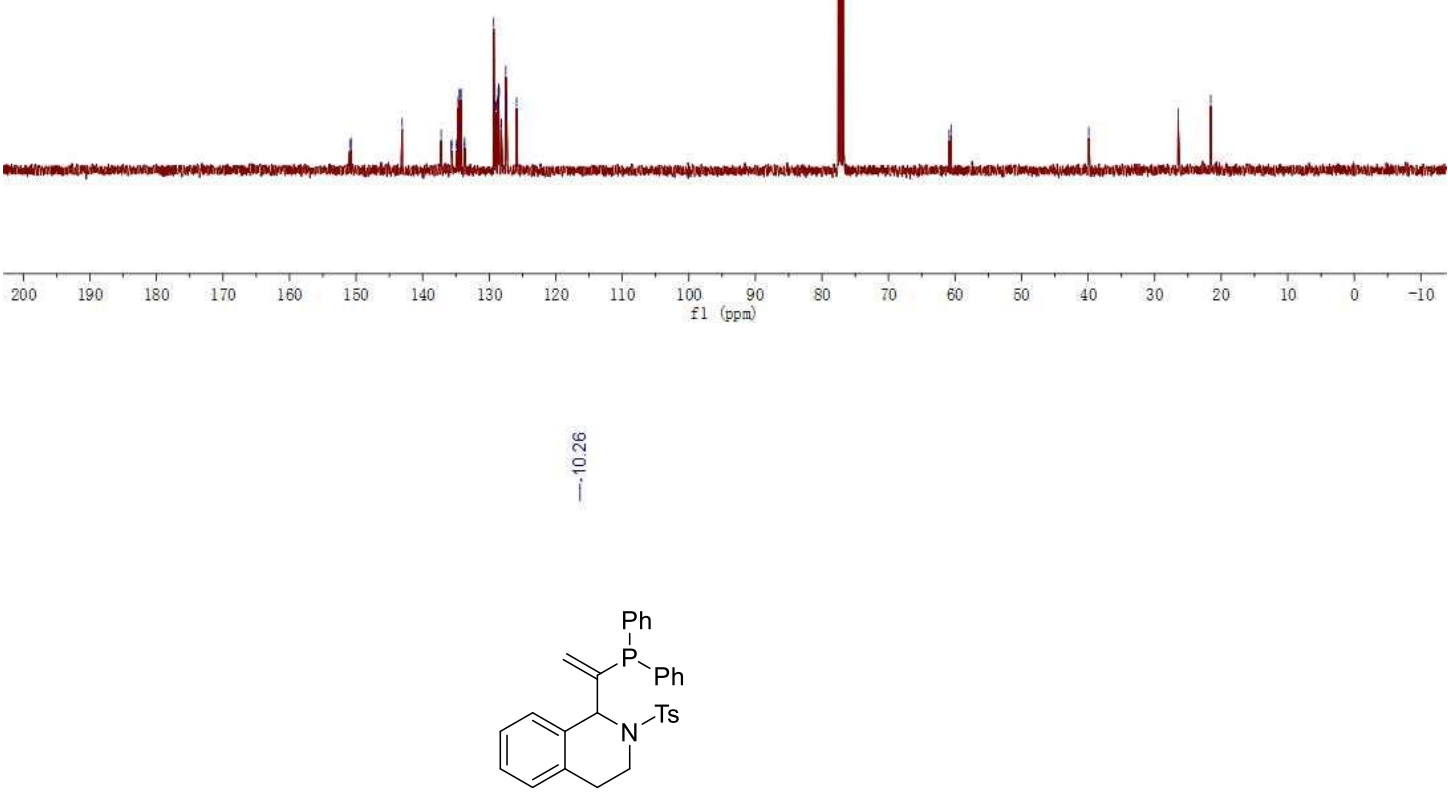

51

${ }^{31} \mathrm{P} \mathrm{NMR}, 162 \mathrm{MHz}, \mathrm{CDCl}_{3}$

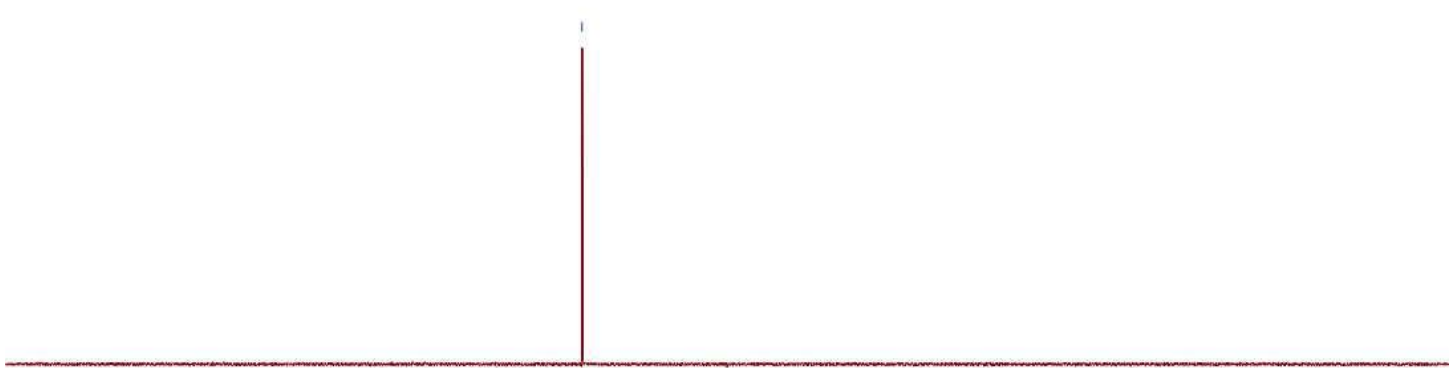

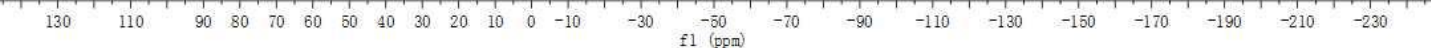




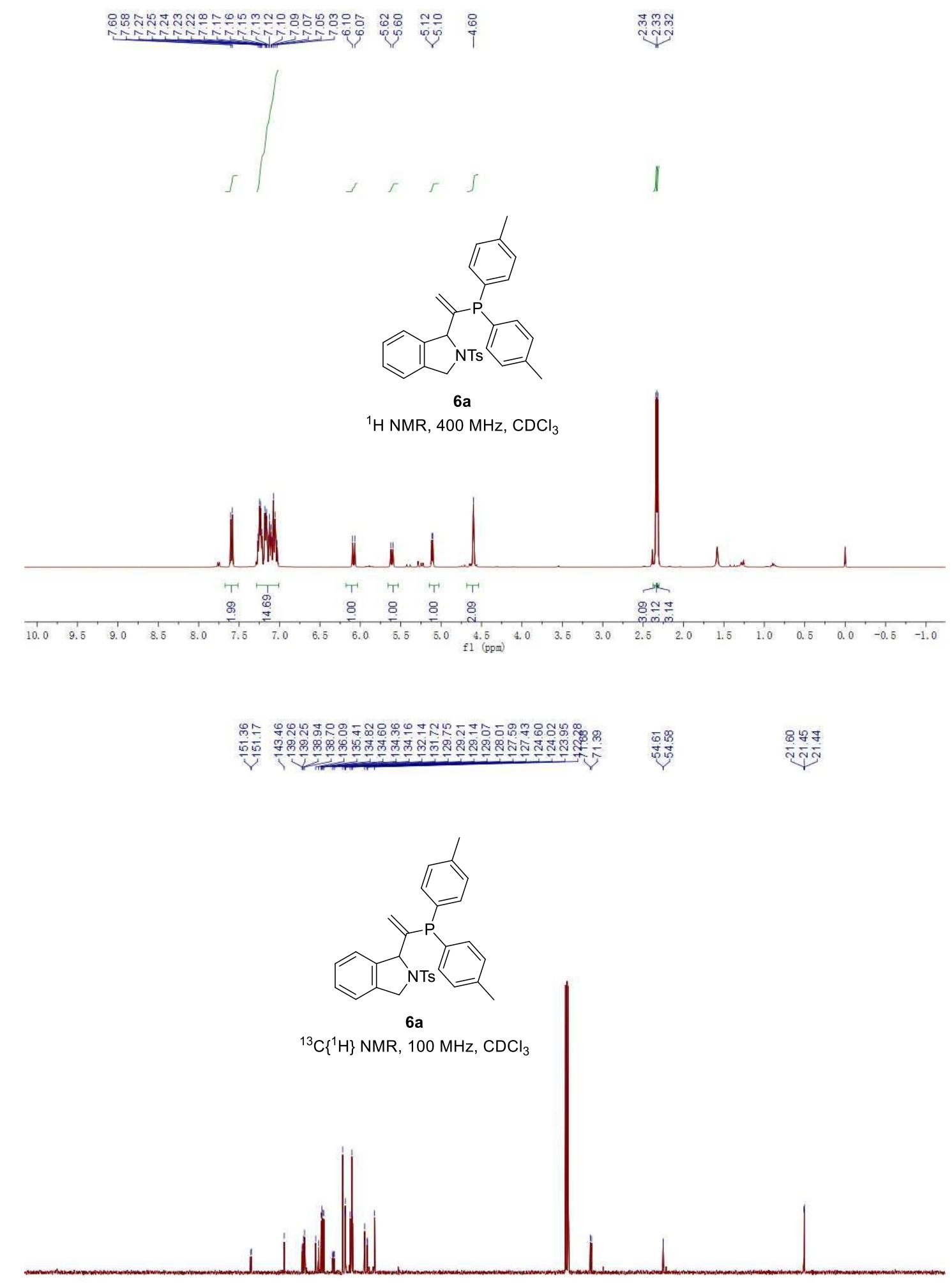

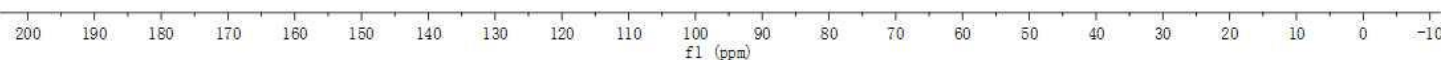




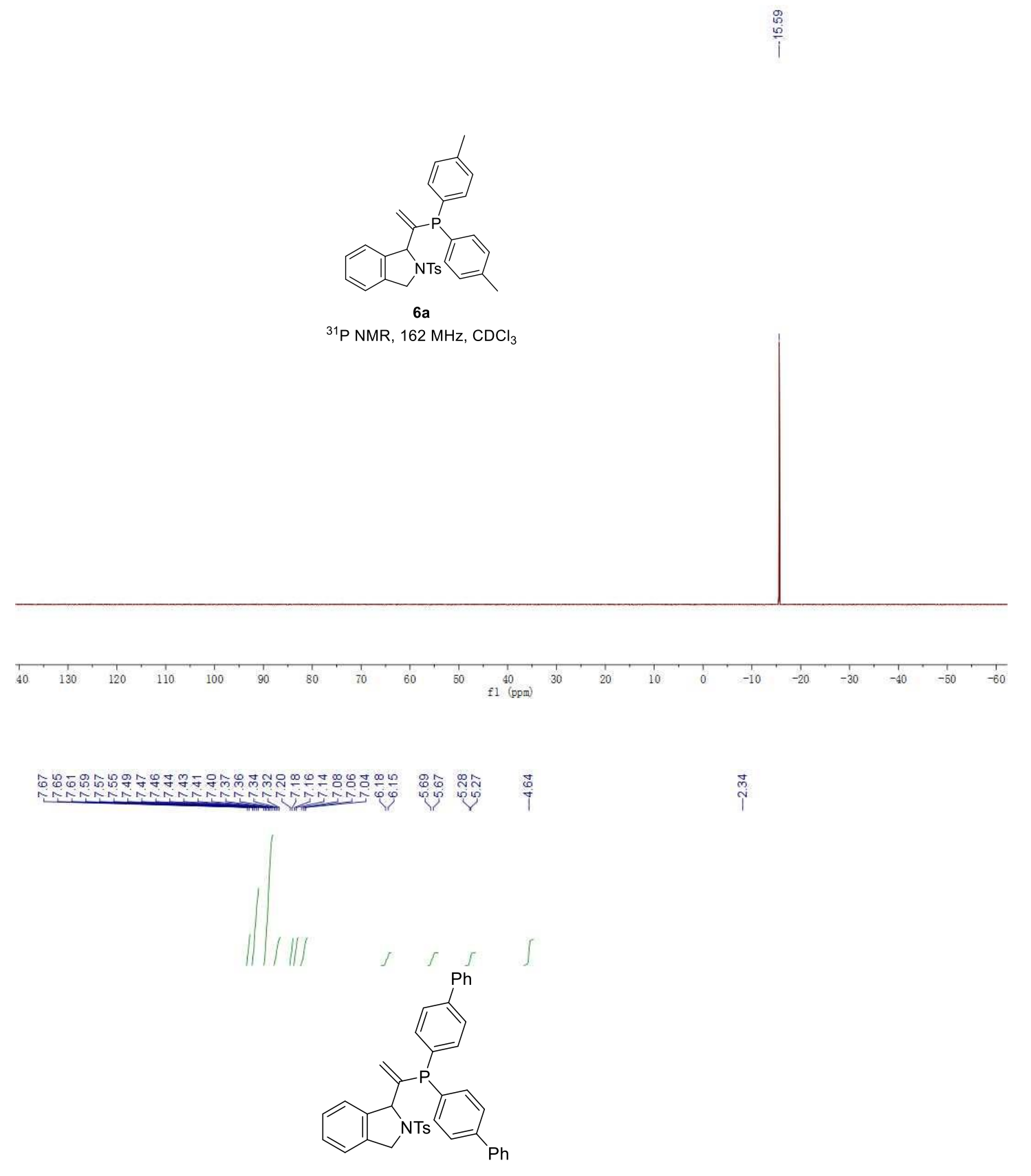

$6 b$

${ }^{1} \mathrm{H} \mathrm{NMR}, 400 \mathrm{MHz}, \mathrm{CDCl}_{3}$

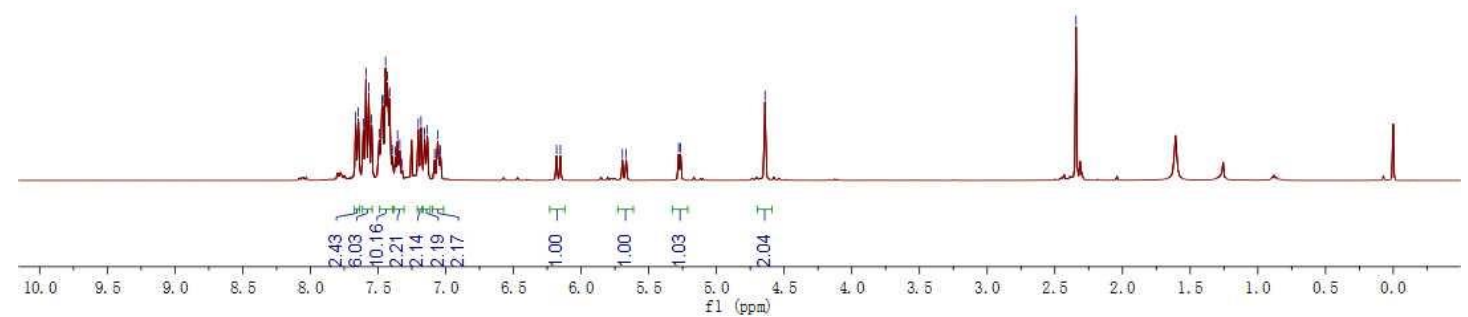



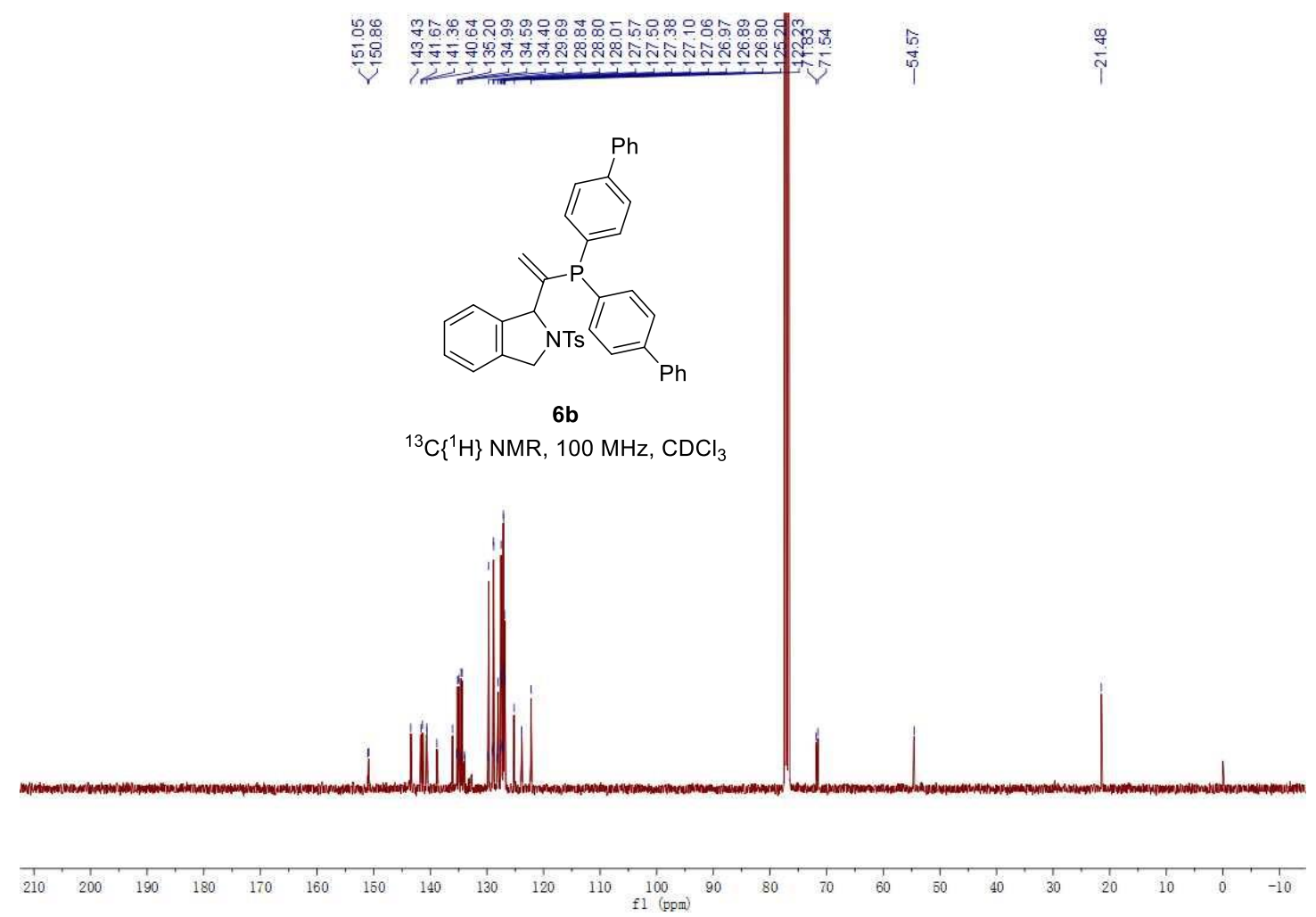

$\frac{\overline{0}}{10}$

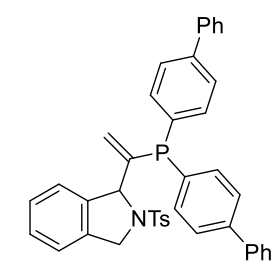

6b

${ }^{31} \mathrm{P} \mathrm{NMR}, 162 \mathrm{MHz}, \mathrm{CDCl}_{3}$

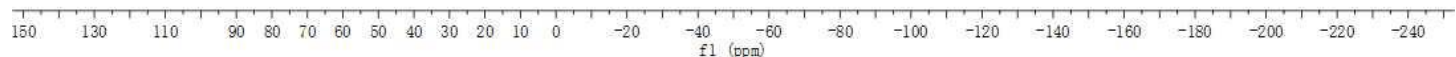




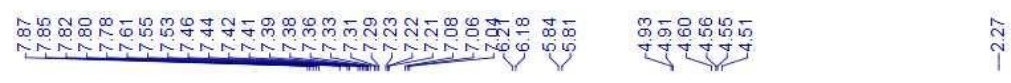

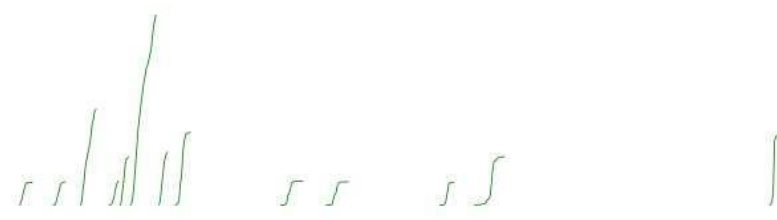<smiles>C=C(C1c2ccccc2C[NH+]1C)P(c1cccc2ccccc12)c1cccc2ccccc12</smiles>

$6 \mathrm{c}$

${ }^{1} \mathrm{H} \mathrm{NMR}, 400 \mathrm{MHz}, \mathrm{CDCl}_{3}$

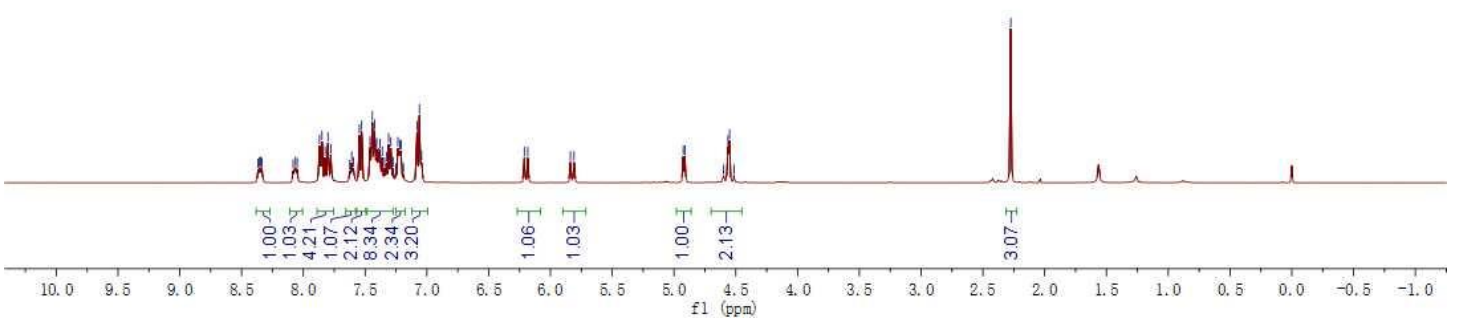

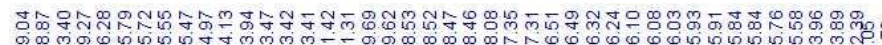

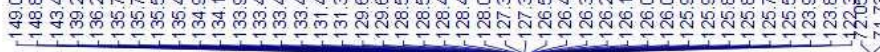

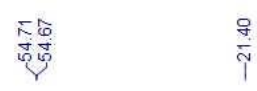

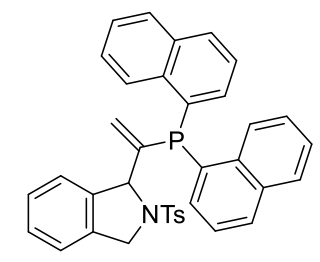

6c

${ }^{13} \mathrm{C}\left\{{ }^{1} \mathrm{H}\right\} \mathrm{NMR}, 100 \mathrm{MHz}, \mathrm{CDCl}_{3}$

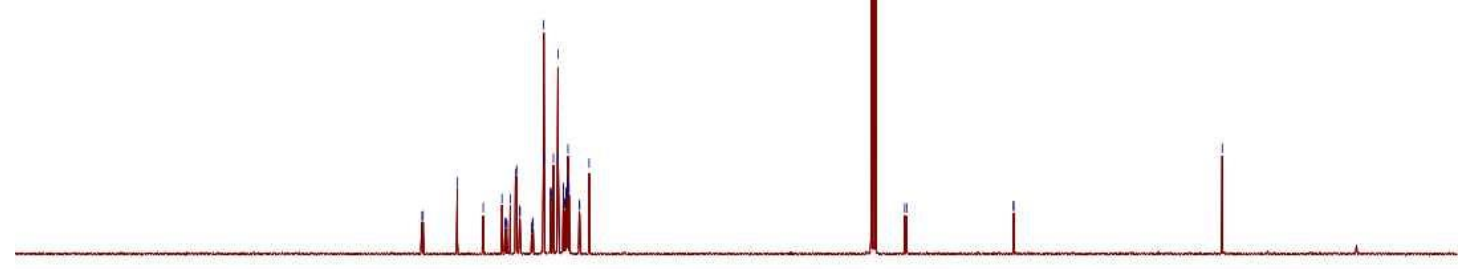

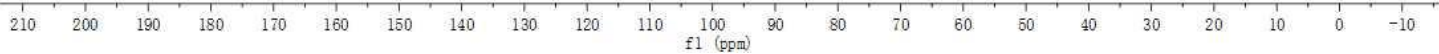




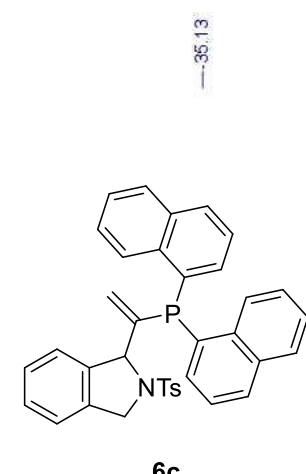

${ }^{31} \mathrm{P}$ NMR, $162 \mathrm{MHz}, \mathrm{CDCl}_{3}$

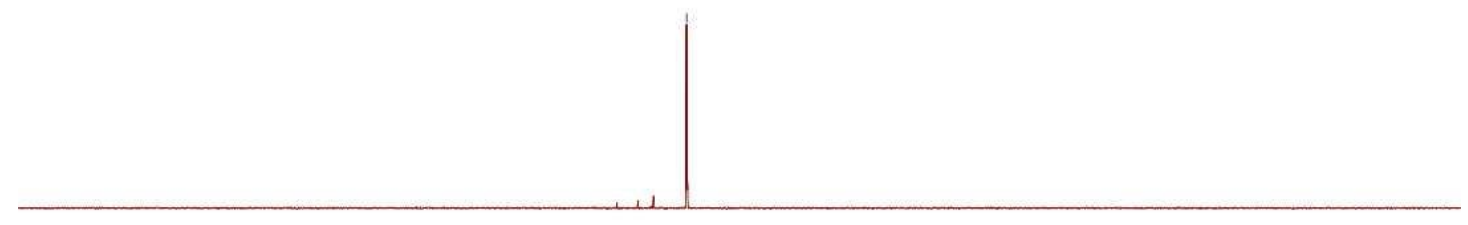

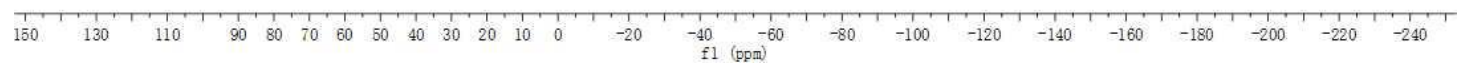

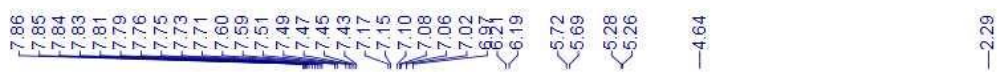
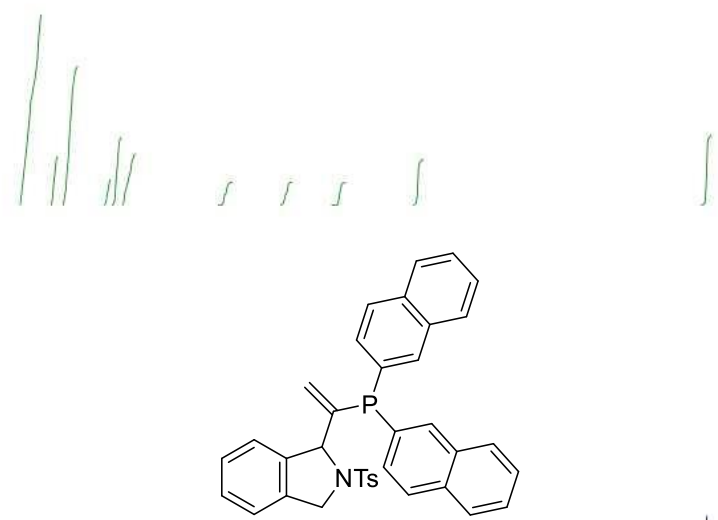

6d

${ }^{1} \mathrm{H} \mathrm{NMR}, 400 \mathrm{MHz}, \mathrm{CDCl}_{3}$

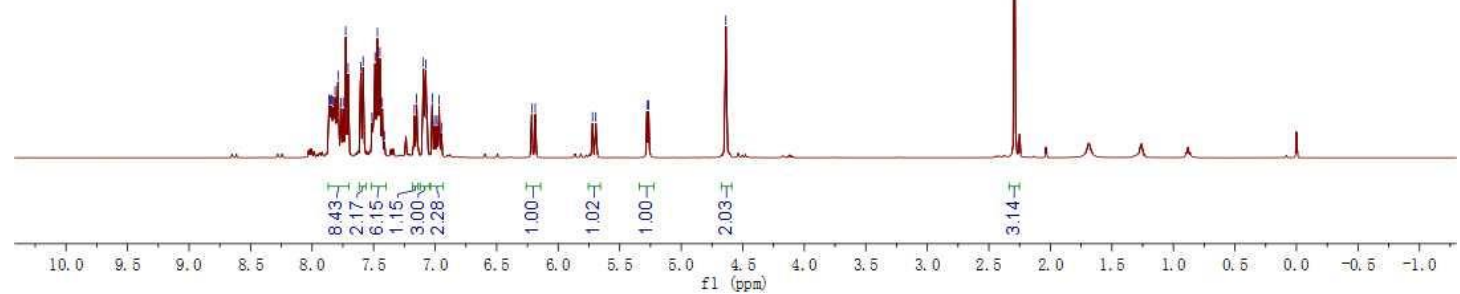




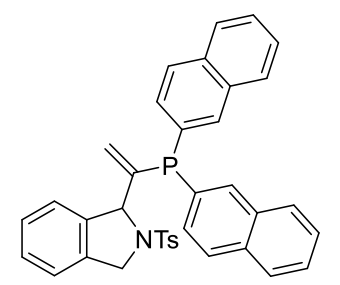

6d

${ }^{13} \mathrm{C}\left\{{ }^{1} \mathrm{H}\right\} \mathrm{NMR}, 100 \mathrm{MHz}, \mathrm{CDCl}_{3}$
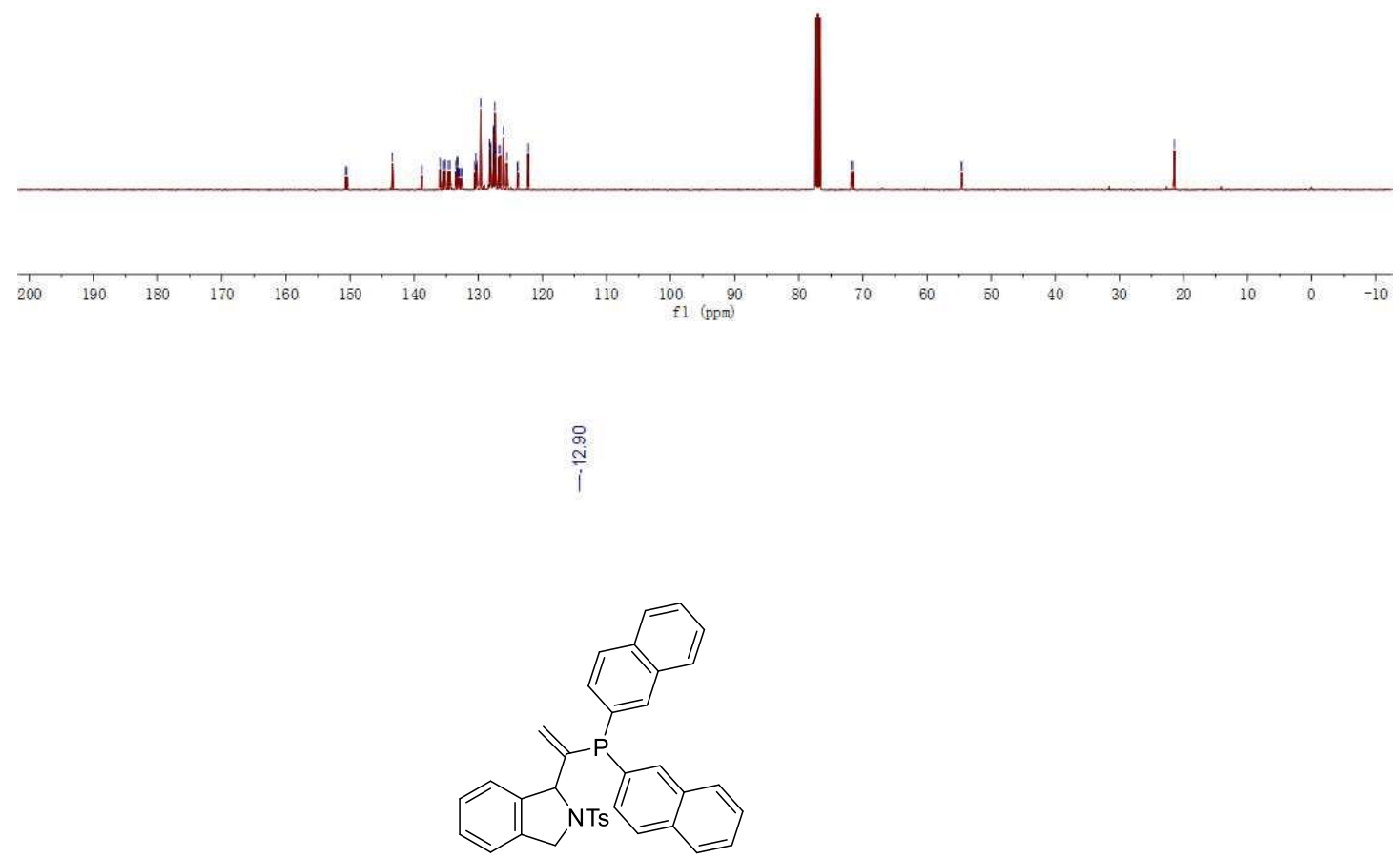

$6 \mathrm{~d}$

${ }^{31} \mathrm{P}$ NMR, $162 \mathrm{MHz}, \mathrm{CDCl}_{3}$

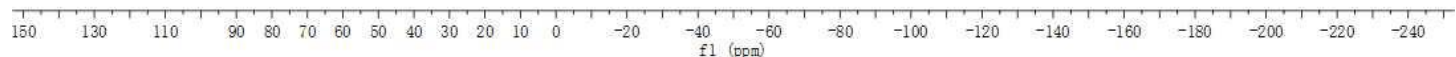




\section{X-ray Crystal Structure and Data of $5 g$}

To get a high quality crystal for X-ray analysis, compound $\mathbf{5 g}$ was dissolved in ethyl acetate and hexane, and was allowed to crystalize via careful evaporation of the solvent. (CCDC 2024402).

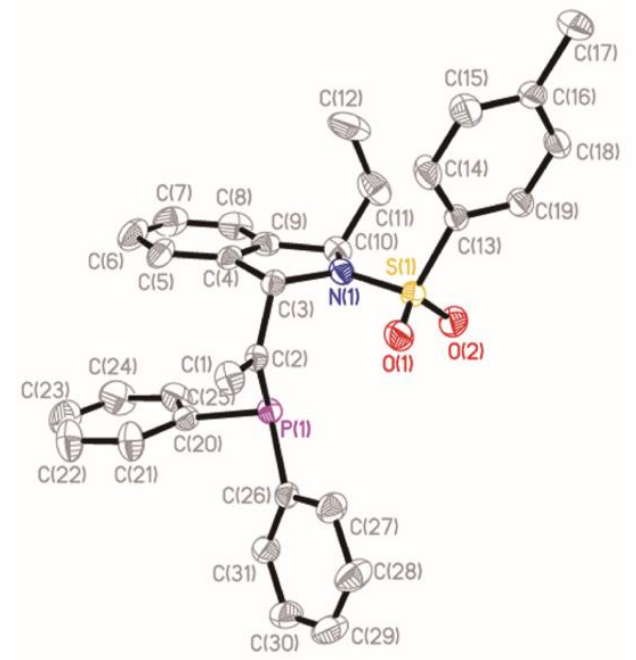

Figure S2. ORTEP drawings of 1,3-anti-5g at 30\% displacement ellipsoid probability (the hydrogen atoms are omitted for clarity).

Table S1. Crystal data and structure refinement for $\mathbf{5 g}$.

\begin{tabular}{|l|l|}
\hline Empirical formula & $\mathrm{C}_{31} \mathrm{H}_{30} \mathrm{NO}_{2} \mathrm{PS}$ \\
\hline Formula weight & 511.59 \\
\hline Temperature & $296(2) \mathrm{K}$ \\
\hline Wavelength & $0.71073 \mathrm{~A}$ \\
\hline Crystal system, space group & Triclinic, P-1 \\
\hline & $\mathrm{a}=6.2227(3) \mathrm{A} \quad$ alpha $=99.244(3) \mathrm{deg}$. \\
\cline { 2 - 3 } & $\mathrm{b}=10.2754(6) \mathrm{A} \quad$ beta $=92.721(3) \mathrm{deg}$. \\
\cline { 2 - 3 } Unit cell dimensions & $\mathrm{c}=21.6046(10) \mathrm{A} \quad$ gamma $=91.579(4) \mathrm{deg}$. \\
\hline Volume & $1361.07(12) \mathrm{A} \mathrm{A}^{\wedge} 3$ \\
\hline Z, Calculated density & $2,1.248 \mathrm{Mg} / \mathrm{m}^{\wedge} 3$ \\
\hline Absorption coefficient & $0.206 \mathrm{~mm}{ }^{\wedge}-1$ \\
\hline F(000) & 540 \\
\hline Crystal size & $0.10 \times 0.10 \times 0.10 \mathrm{~mm}$ \\
\hline
\end{tabular}




\begin{tabular}{|l|l|}
\hline Theta range for data collection & 2.01 to 25.00 deg. \\
\hline Limiting indices & $-7<=\mathrm{h}<=7,-11<=\mathrm{k}<=12,-25<=1<=24$ \\
\hline Reflections collected / unique & $10999 / 4771[\mathrm{R}(\mathrm{int})=0.0253]$ \\
\hline Completeness to theta $=25.00$ & $99.4 \%$ \\
\hline Absorption correction & None \\
\hline Refinement method & Full-matrix least-squares on $\mathrm{F}^{\wedge} 2$ \\
\hline Data / restraints / parameters & $4771 / 6 / 313$ \\
\hline Goodness-of-fit on F^2 & 1.041 \\
\hline Final R indices [I>2sigma(I)] & $\mathrm{R} 1=0.0446, \mathrm{wR} 2=0.1294$ \\
\hline R indices (all data) & $\mathrm{R} 1=0.0568, \mathrm{wR} 2=0.1377$ \\
\hline Largest diff. peak and hole & 0.603 and $-0.372 \mathrm{e} . \mathrm{A}^{\wedge}-3$ \\
\hline
\end{tabular}

